\title{
Geometric Nonlinear Finite Element Analysis of Active Fibre Composite Bimorphs
}

\author{
By \\ Robert Kernaghan \\ A thesis submitted to \\ The Faculty of Graduate Studies and Research \\ in partial fulfilment of \\ the degree requirements of \\ Master of Applied Science \\ Ottawa-Carleton Institute for \\ Mechanical and Aerospace Engineering \\ Department of Mechanical and Aerospace Engineering \\ Carleton University \\ Ottawa, Ontario, Canada \\ November 2011 \\ Copyright $(\mathcal{C}$ \\ 2011 - Robert Kernaghan
}


Library and Archives

Canada

Published Heritage

Branch

395 Wellington Street

Ottawa ON K1A ON4

Canada
Bibliothèque et

Archives Canada

Direction du

Patrimoine de l'édition

395 , rue Wellington

Ottawa ON K1A ON4

Canada
Your file Votre référence

ISBN: 978-0-494-87798-2

Our file Notre référence

ISBN: $978-0-494-87798-2$

\section{NOTICE:}

The author has granted a nonexclusive license allowing Library and Archives Canada to reproduce, publish, archive, preserve, conserve, communicate to the public by telecommunication or on the Internet, loan, distrbute and sell theses worldwide, for commercial or noncommercial purposes, in microform, paper, electronic and/or any other formats.

The author retains copyright ownership and moral rights in this thesis. Neither the thesis nor substantial extracts from it may be printed or otherwise reproduced without the author's permission.
AVIS:

L'auteur a accordé une licence non exclusive permettant à la Bibliothèque et Archives Canada de reproduire, publier, archiver, sauvegarder, conserver, transmettre au public par télécommunication ou par l'Internet, prêter, distribuer et vendre des thèses partout dans le monde, à des fins commerciales ou autres, sur support microforme, papier, électronique et/ou autres formats.

L'auteur conserve la propriété du droit d'auteur et des droits moraux qui protege cette thèse. $\mathrm{Ni}$ la thèse ni des extraits substantiels de celle-ci ne doivent être imprimés ou autrement reproduits sans son autorisation.
In compliance with the Canadian Privacy Act some supporting forms may have been removed from this thesis.

While these forms may be included in the document page count, their removal does not represent any loss of content from the thesis.
Conformément à la loi canadienne sur la protection de la vie privée, quelques formulaires secondaires ont été enlevés de cette thèse.

Bien que ces formulaires aient inclus dans la pagination, il n'y aura aucun contenu manquant. 


\section{Abstract}

Active fibre composite-actuated bimorphic actuators were studied in order to measure deflection performance. The deflection of the actuators was a function of the actuating electric potential applied to the active material as well as the magnitude of the axial preload applied to the bimorphic structure. This problem required the use of geometric nonlinear modeling techniques.

Geometric nonlinear finite element analysis was undertaken to determine the deflection performance of Macro Fibre Composite (MFC)- and Hollow Active Fibre (HAFC)-actuated bimorphic structures. A physical prototype MFC-actuated bimorphic structure was manufactured in order to verify the results obtained by the finite element analysis.

Theses analyses determined that the bimorphic actuators were capable of significant deflection. The analyses determined that the axial preload of the bimorphic actuators significantly amplified the deflection performance of the bimorphic actuators. The deflection performance of the bimorphic actuators suggest that they could be candidates to act as actuators for the morphing wing of a micro unmanned air vehicle. 


\section{Table of Contents}

Abstract

Table of Contents iv

List of Tables

List of Figures viii

Nomenclature $\quad$ xi

Symbols $\quad$ xii

Chapter 1: Introduction 1

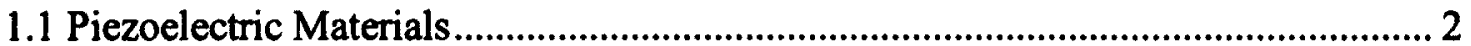

1.2 Piezoelectric Actuators ............................................................................... 11

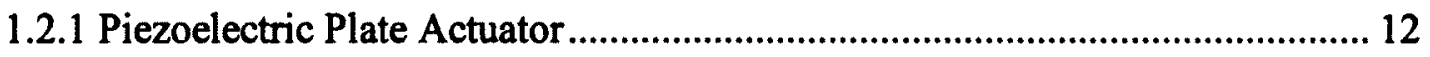

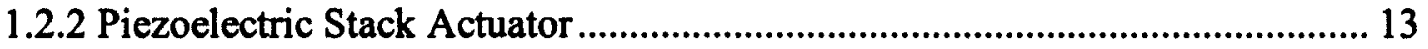

1.2.3 Interdigitated Electrode Monolithic Actuator .......................................... 15

1.2.4 Active Fibre Composite (AFCs) and Macro Fibre Composites (MFCs) ......... 16

1.2.5 Hollow Active Fibre Composite Actuator ................................................. 20

1.2.6 Unimorphic and Bimorphic Actuators ........................................................ 24

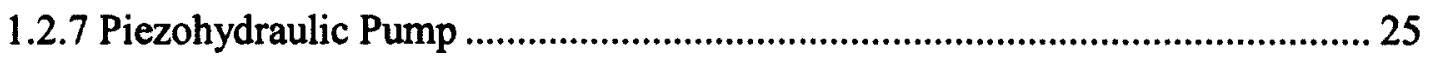

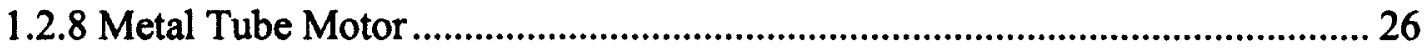

1.2.9 Feed-Screw Piezoelectric Actuator............................................................ 27

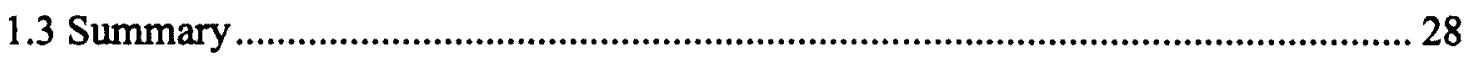

Chapter 2: Nonlinear Finite Element Analysis 29

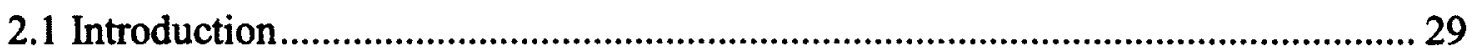

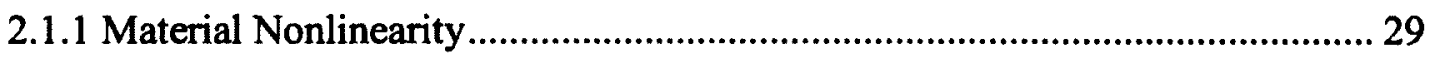

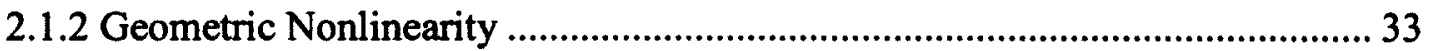


2.1.3 Load Nonlinearity or Boundary Condition Nonlinearity .................................... 40

2.2 Finite Element Analysis (FEA)........................................................................ 40

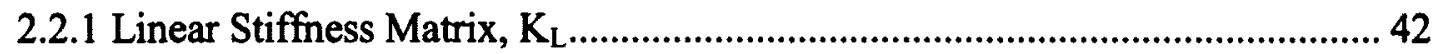

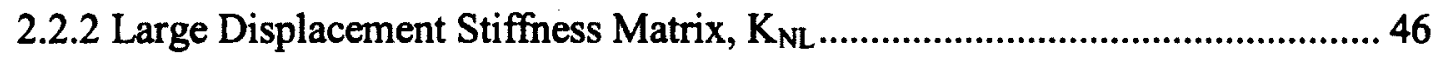

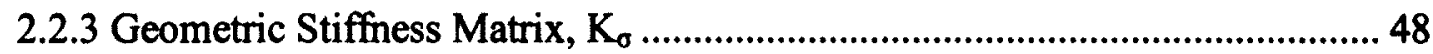

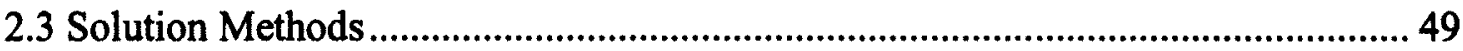

2.3.1 Incremental (Euler) Method............................................................................... 49

2.3.2 Iterative Method (Newton-Raphson) Method................................................... 51

2.3.3 Corrected Incremental Method .......................................................................52

Chapter 3: Validation $\quad 55$

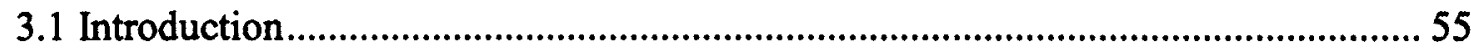

3.2 Linear Elastic Problems: MacNeal and Harder Tests ............................................. 55

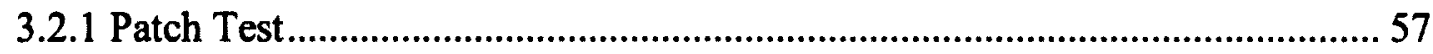

3.2.2 Straight Beam Test......................................................................................... 59

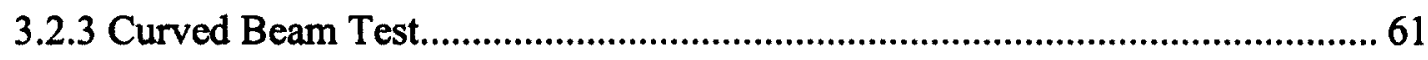

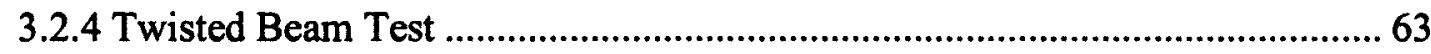

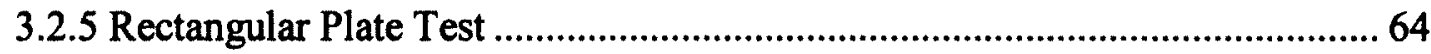

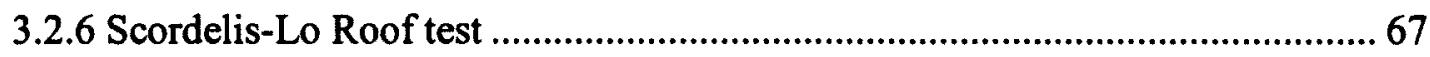

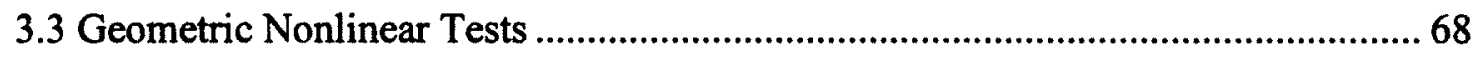

3.3.1 Heavily Loaded Flat Beam Test ..................................................................... 69

3.3.2 Dually-Loaded Beam Test .............................................................................. 71

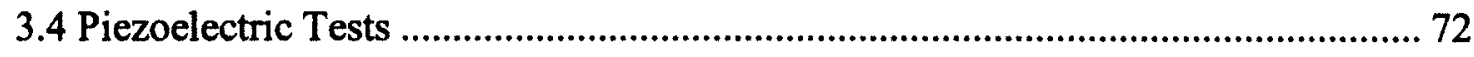

3.4.1 Piezoelectrically-Extended Straight Beam Test................................................ 73

3.4.2 Piezoelectrically-Sheared Straight Beam Test.................................................. 74

3.4.3 Piezoelectrically-Actuated Bimorphic Beam Test.............................................. 76

3.4.4 Piezoelectric Hollow Active Fibre test ...................................................... 78

3.4.5 Piezoelectric Hollow Active Fibre Composite (HAFC) Test ………………..... 79

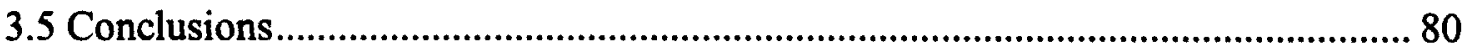

$\begin{array}{lc}\text { Chapter 4: Analysis and Results } & 81\end{array}$ 


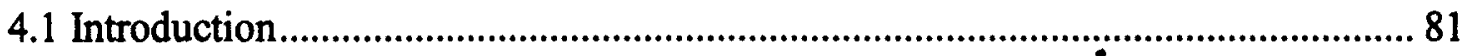

4.2 Macro Fibre Composite-Actuated Bimorphic Structure....................................... 83

4.3 Finite Element Model of Macro Fibre Composite-Actuated Bimorphic Structure 88

4.4 Finite Element Model of Hollow Active Fibre Composite (HAFC) Actuator ....... 94

4.4.1 HAFC Actuator Reliability During Fibre Cracking .................................... 94

4.4.2 HAFC Sensor Reliability During Fibre Cracking........................................... 98

4.4.3 Finite Element Analysis of Hollow Active Fibre Composite-Actuated

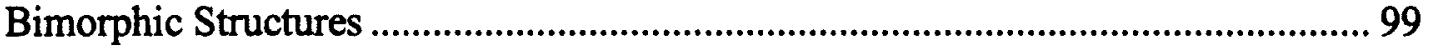

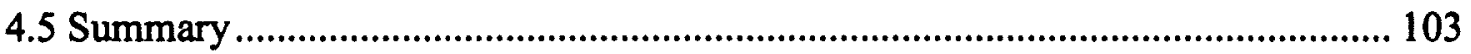

$\begin{array}{ll}\text { Chapter 5: Conclusions } & 105\end{array}$

$\begin{array}{ll}\text { Chapter 6: References } & 108\end{array}$ 


\section{List of Tables}

Table 1-1: Material and piezoelectric properties of PZT-4 [26]........................................ 10

Table 2-1: Weight factors for 20-node quadratic brick element C3D20 [44]................... 45

Table 3-1: Results for patch test ...................................................................................... 58

Table 3-2: Results for the straight beam test .................................................................. 59

Table 3-3: Results for the curved beam test....................................................................... 62

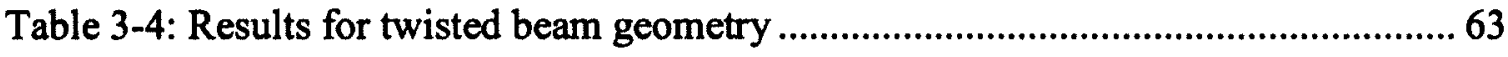

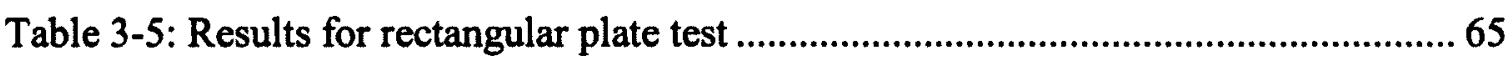

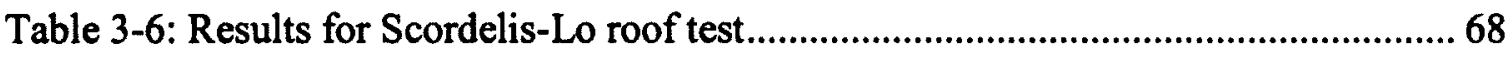

Table 3-7: Results for dually-loaded beam test ................................................................ 72

Table 3-8: results for piezoelectrically-extended straight beam test .................................. 74

Table 3-9: Results for piezoelectric sheared straight beam test........................................ 75

Table 3-10: Results for piezoelectrically-actuated bimorphic beam test........................... 77

Table 4-1: Material parameters of the bimorphic structure ................................................ 88

Table 4-2: Material properties of active fibre composite bimorph structure ..................... 91

Table 4-3: Characteristics of MFC- and HAFC-actuated bimorphs................................. 101 


\section{List of Figures}

Figure 1-1: Atomic structure of PZT-5H (Lead Zirconate Titanate) [25] ......................... 3

Figure 1-2: Material poling and the reorientation of Weiss domains [25] ....................... 5

Figure 1-3: Strain vs. electric field during poling [26] ..................................................6

Figure 1-4: Constitutive Equation Forms [28] ....................................................... 10

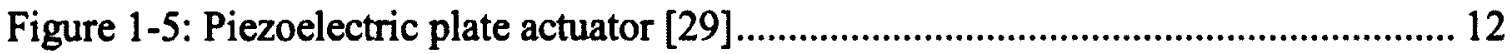

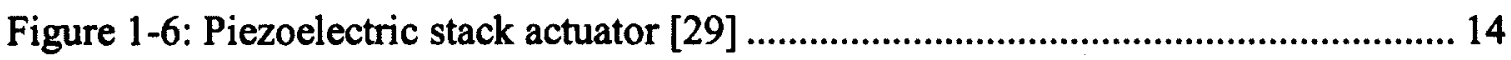

Figure 1-7: Interdigitated electrode monolithic actuator [30].................................... 16

Figure 1-8: Active Fibre Composite (AFC) actuator [31] ......................................... 17

Figure 1-9: Electric field flow through AFC (left) and MFC (right) (adapted from [30]) 18

Figure 1-10: Piezoelectric fibre extrusion process....................................................... 19

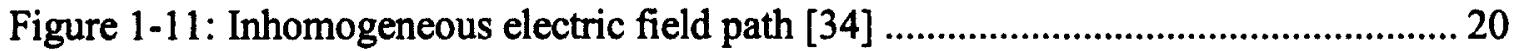

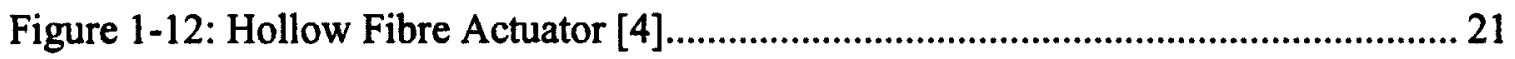

Figure 1-13: Hollow Piezoceramic Fibre Coextrusion Process [36] .............................. 24

Figure 1-14: Unimorphic (left) and bimorphic (right) structures ................................. 25

Figure 1-15: Piezohydraulic pump [37] …................................................................ 26

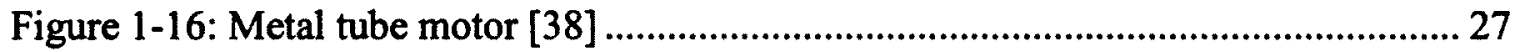

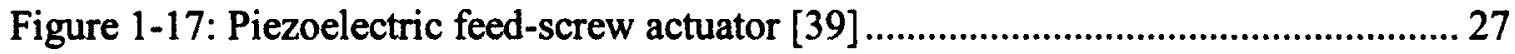

Figure 2-1: Examples of material response [40] .......................................................... 30

Figure 2-2: Stain vs. electric behaviour for various piezoelectric materials [26]............. 31

Figure 2-3: Typical strain vs. electric field hysteresis in piezoelectric materials [26] ..... 33

Figure 2-4: Structure subjected to a stress, resulting in large strain deformation............. 34

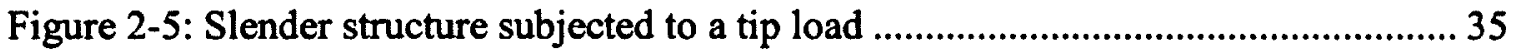

Figure 2-6: Displacement resistance of slender beam subjected to tip load ................... 37

Figure 2-7: Pinned beam subjected to axial point loads and lateral pressure load [42].... 39

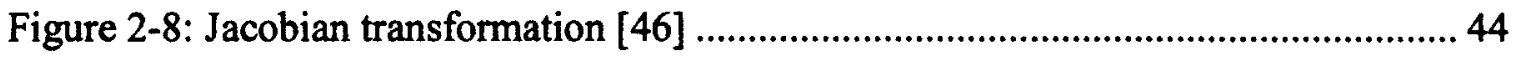


Figure 2-9: Gaussian integration scheme [47] ............................................................. 45

Figure 2-10: Initial configuration, $x^{0}$ and deformed configuration, $x$ [43] ...................... 46

Figure 2-11: Incremental (Euler) method [48] .................................................................. 50

Figure 2-12: Newton-Raphson method [48] ............................................................... 52

Figure 2-13: Incremental method with load correction [48].............................................. 54

Figure 3-1: Patch test geometry and nodal coordinates [50] ............................................. 57

Figure 3-2: Straight beam, with ........................................................................................ 59

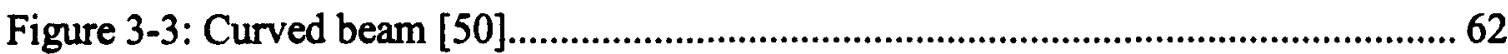

Figure 3-4: Twisted beam [50] ....................................................................................63

Figure 3-5: Plate geometry: (left) aspect ratio 1.0 and (right) aspect ratio 5.0 [50] .........65

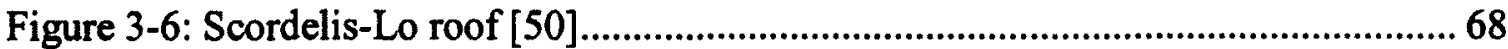

Figure 3-7: Flat beam with 16 elements (left) and displacement resulting from load...... 70

Figure 3-8: Results for heavily loaded flat plate ................................................................... 70

Figure 3-9: Dually-loaded beam ................................................................................... 71

Figure 3-10: Straight beam undergoing piezoelectric extension [44]............................. 74

Figure 3-11: Straight beam undergoing piezoelectric shear [44].................................... 75

Figure 3-12: bimorphic beam undergoing piezoelectric deformation [44]...................... 77

Figure 3-13: Actuated quarter-fibre ................................................................................ 79

Figure 3-14: One quarter of a unit cell of the hollow active fibre composite.................... 80

Figure 4-1: Macro Fibre Composite (MFC)-based morphing wing [54]........................... 82

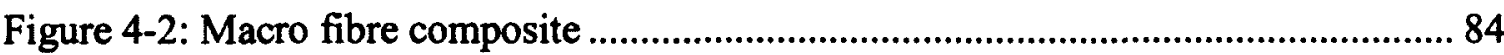

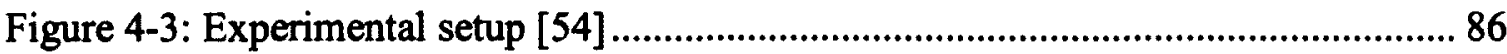

Figure 4-4: Composite beam undergoing applied load and piezoelectric stresses [53].... 87

Figure 4-5: Finite element model of the MFC-actuated bimorphic structure.................... 89

Figure 4-6: Cross-section of the finite element model....................................................... 90

Figure 4-7: Load conditions applied to active fibre bimorph structure ............................. 92

Figure 4-8: Tip deflection of an MFC-actuated bimorph .................................................. 93

Figure 4-9: Finite element model of HAFC lamina [8] ................................................ 95

Figure 4-10: Hollow active fibre with electrode placement and poling ............................ 96

Figure 4-11: Broken fibre effects [8] .............................................................................. 97 
Figure 4-12: Lamina extension performance with introduction of fibre cracks [8] ......... 97

Figure 4-13: Lamina sensing performance with introduction of fibre cracks [8]............. 98

Figure 4-14: Voltage distribution on damaged sensor lamina [8] ..................................... 99

Figure 4-15: Cross-section of the HAFC-actuated bimorphic structure............................ 100

Figure 4-16: Electric potential distribution of HAFC-actuated bimorph......................... 102

Figure 4-17: Out-of-plane tip deflection of HAFC-actuated bimorph............................. 103 


\section{Nomenclature}

AFC Active Fibre Composite

EAP Electroactive Polymer

FEA Finite Element Analysis

FEM Finite Element Method

HAFC Hollow Active Fibre Composite

IDE Interdigitated Electrodes

MAV Micro Air Vehicle

$\mathrm{M}+\mathrm{H} \quad \mathrm{MacNeal}$ and Harder

MFC Macro Fibre Composite

PVDF Polyvinyldiene Difluoride

PZT Lead Zirconate Titanate

VSSP Viscous Suspension Spinning Process 


\section{Symbols}

$1,2,3$ Principal coordinates
A Area
c Compliance
D Electric displacement
d Piezoelectric Coupling Coefficient (Strain-Charge)
$\bar{E} \quad$ Electric Field
e Piezoelectric Coupling Coefficient (Stress-Charge)
E Young's Modulus, Elastic Modulus
F Deformation Gradient
g Piezoelectric Coupling Coefficient (Strain-Voltage)
r Residual Load
G Shear Modulus
I Second Moment of Area
K Stiffness Matrix
L Length
P Pressure
q Piezoelectric Coupling Coefficient (Stress-Voltage)
S Strain 


$\begin{array}{ll}\mathbf{s} & \text { Stiffness } \\ \hat{s} & \text { Piola-Kirchoff Stress } \\ \text { Tmp } & \text { Temperature } \\ \mathrm{T} & \text { Stress } \\ \mathrm{t} & \text { Thickness } \\ \mathrm{u}, \mathrm{v}, \mathrm{w} & \text { Displacements in } \mathrm{x}, \mathrm{y}, \mathrm{z}, \text { respectively } \\ \mathrm{x}, \mathrm{y}, \mathrm{z} & \text { Cartesian coordinates } \\ \varepsilon & \text { Permittivity } \\ \varepsilon_{0} & \text { Permittivity of Vacuum } \\ \mu & \text { Poisson's Ratio }\end{array}$




\section{Chapter 1: Introduction}

Actuators are mechanical devices that translate energy into a prescribed motion. Conventional actuators are typically mechanical actuators, electro-mechanical actuators, pneumatic actuators or hydraulic actuators. However, these actuators can be impractical for applications that require an extremely high degree of precision. Conventional actuators are composed of precisely manufactured components. It is difficult for conventional actuators to deliver precise motion at small scales. Piezoelectric actuators, coupled with sensors generate a feedback that allows for active control. This active control allows piezoelectric actuators to deliver precise motion at small scales. Piezoelectric actuators are therefore suited for small scale applications.

There are some operations that require high actuation power density (actuation power with respect to weight). Some piezoelectric actuators feature much higher power densities as compared with conventional actuators. The deliverable mechanical energy of piezoelectric actuators can be as much as ten times higher per unit mass as compared to comparable conventional actuators [1].

Micro Air Vehicles (MAVs) are one example of operations that require high actuation power density. Piezoelectric actuators are of interest in the field of MAVs as they can replace many conventional acutators. 
The behaviour of piezoelectric actuators is examined in this thesis with the assistance of Finite Element Analysis (FEA). The piezoelectric actuators in question undergo large deformations, producing nonlinear deformation effects. As such, the large deformations undergone are described as geometric nonlinear deformation. Therefore, the nonlinear finite element model (FEM) is used in order to take into account these large, geometric nonlinear deformations.

\subsection{Piezoelectric Materials}

Piezoelectric materials are characterized by the ability to generate electric field when subjected to mechanical stress. This ability is referred to as the piezoelectric effect. Conversely, piezoelectric materials also exhibit the inverse piezoelectric effect (the production of mechanical strain when subjected to electric field).

Piezoelectric materials have been used in order to actuate active structures $[2,3,4,5]$, to pump fluid $[6,7]$, to sense strains $[8,9]$, to generate ultrasonic waves in structural health monitoring of components $[10,11,12]$ to provide active motion or vibration control to structures $[13,14,15,16]$, in noise suppression [17], to create and detect sound $[18,19]$ and to generate electricity in energy harvesting applications $[20,21,22,23]$.

Crystalline materials are composed of many repeating atomic cells such as those illustrated in Figure 1-1. The properties of materials are determined largely by the structure of these cells. The atomic cell of piezoelectric materials contains an electric dipole that can be influenced by applied stresses, producing charge displacement. 
Likewise, electric fields applied to the material influence the dipole, creating material strain.

(a)

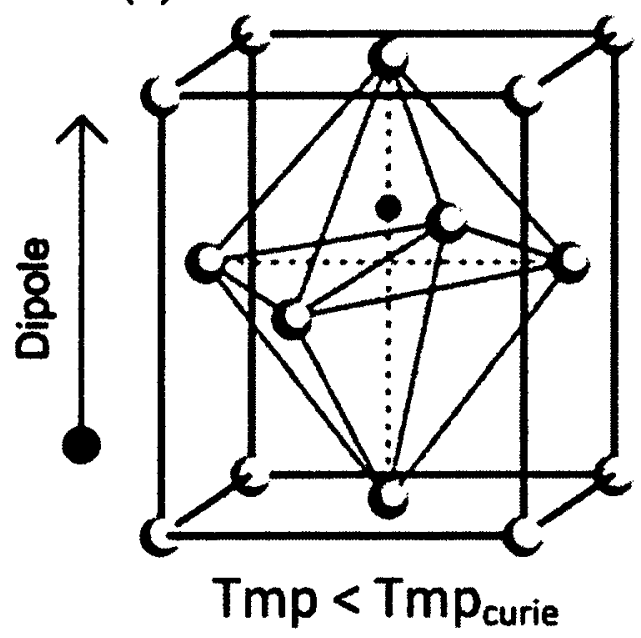

(b)

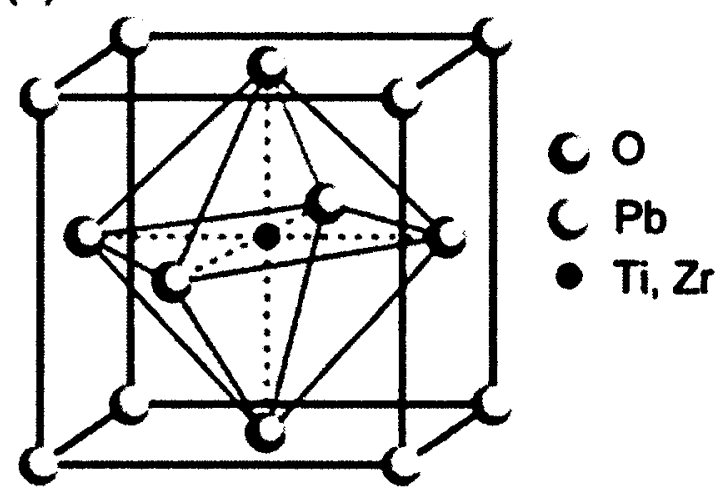

Tmp $>$ Tmp $_{\text {curie }}$

Figure 1-1: Atomic structure of PZT-5H (Lead Zirconate Titanate) [25]

(a) Below the Curie Temperature (Tmp $\left.\mathrm{Purre}_{\mathrm{e}}\right)$ : Electric Dipole and Piezoelectric Effect

(b) Above the Curie Temperature: Symmetric, No Dipole

The piezoelectric effect is only exhibited over a limited range of temperatures. The Curie temperature is the upper bound of this temperature range. Below the Curie temperature, the positive and negative centres of charge in the cell have an equilibrium location away from each other, producing magnetic asymmetry, and hence the piezoelectric effect is exhibited. Above this range, the equilibrium locations of charge coincide, and the piezoelectric effect is no longer exhibited [24].

The effect of the Curie temperature is illustrated in Figure 1-1. Below the Curie temperature in Figure 1-1 (a), the cell contains non-coincident centres of charge, which create an electric dipole. This dipole (oriented along the direction of the arrow) can be 
influenced by an external electric field and thus exhibits the piezoelectric effect. Above the Curie temperature (illustrated in Figure 1-1 (b)), the centres of charge become coincident. No dipole is created and the piezoelectric effect is not present.

Volumes of material with similarly oriented dipoles are called Weiss domains [25]. A material may contain many Weiss domains. Figure 1-2(a) illustrates a material containing many, randomly oriented Weiss domains (The dipole orientations of the Weiss domains are denoted as arrows). If the Weiss domains within a material form a discernible net orientation (polarization), then the material will exhibit the piezoelectric phenomenon. The piezoelectric phenomenon can be brought about through the poling of the material. Poling consists of reorienting the Weiss with the application of a strong poling electric field. Weiss domains will reorient themselves to align with the poling electric field. Figure 1-2(b) illustrates the reorientation of the Weiss domains within the material in response to poling electric field. Once the poling is complete and the poling electric field is removed, the Weiss domains of the material retain a net dipole orientation and cannot easily return to their original orientations. Figure 1-2(c) illustrates the orientations of the Weiss domains in the newly poles material. The poled material can then be influenced by external electric fields and is said to exhibit the piezoelectric phenomenon. [26] The Weiss domains within the material share a net orientation. The material can be acted upon by external electric fields and is said to exhibit the piezoelectric phenomenon. 


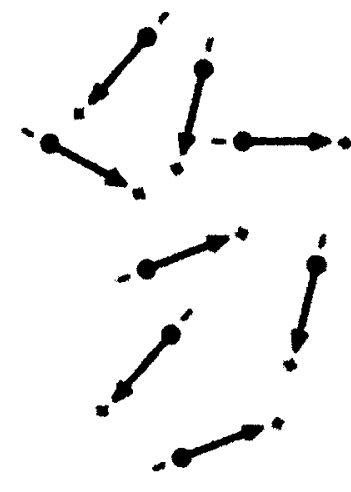

(a)

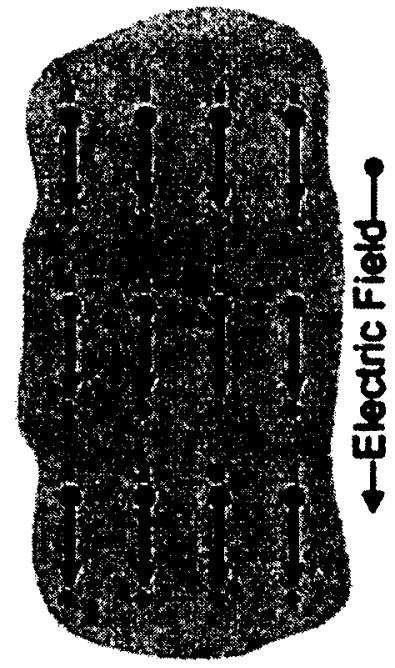

(b)

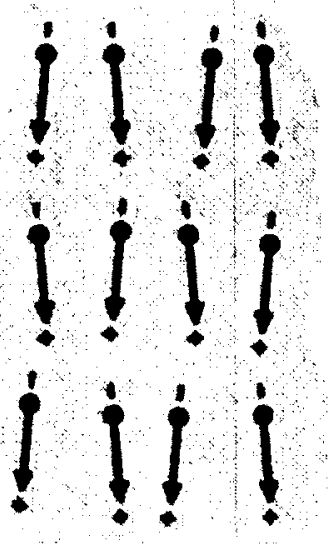

(c)

Figure 1-2: Material poling and the reorientation of Weiss domains [25]

(a) Randomly oriented Weiss domains, no net pole direction

(b) Poling: Weiss domains align to electric field

(c) Poled material aligned Weiss domains exhibits the piezoelectric phenomenon

The relationship between the intensity of the piezoelectric effect and the degree of poling is observed throughout the poling process. The poling process is illustrated in Figure 1-3. Figure 1-3(a) illustrates a material that is poled in the downwards direction. The Weiss domains of the material are all oriented in a similar direction. The poling electric field (oriented upward) is applied in Figure 1-3(b). A (negative, contracting) strain in the material is observed as a result of the electric field applied in the (upward) direction opposite to that of the Weiss domain orientation (downward). The Weiss domains reorient themselves in order to align with the electric field. The Weiss domains are reoriented (upward) in Figure 1-3(c). It should be noted that not all orientations of 
Weiss domains are achievable within polycrystalline materials. In polycrystalline materials, the Weiss domains will reach a net equilibrium orientation nearing that of the poling field. As such, the Weiss domains will not be uniformly oriented. However, a net Weiss domain dipole orientation will be reached that will near the poling field orientation. Figure 1-3(d) illustrates the application of an electric field oriented (upwards) in the same direction of the material poling direction (also upwards). The material will exhibit a (positive) strain in the presence of the electric field. [26]

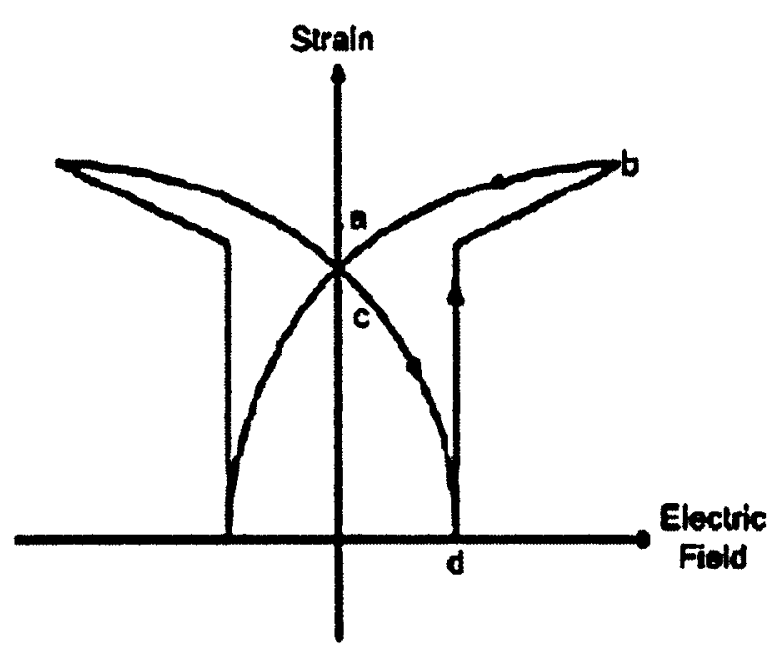

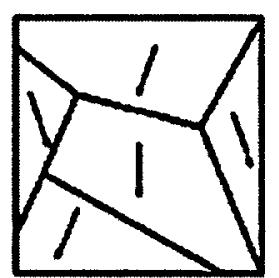

(a) $E=0$

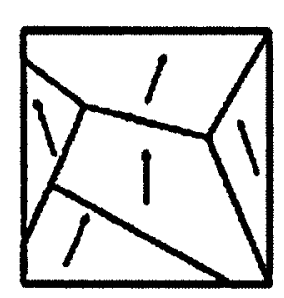

(c) $E=0$

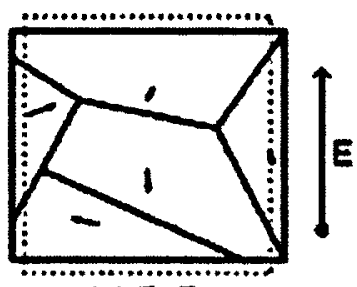

(b) $E=E c$

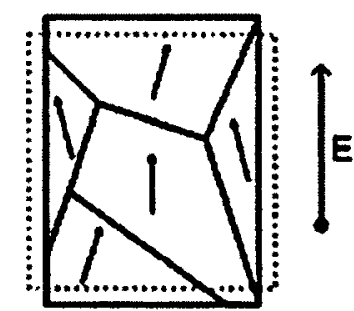

(d) $E=E_{\text {max }}$

Figure 1-3: Strain vs. electric field during poling [26]

(a) Piezoelectric material poled in the downward direction

(b) Weiss domains reorient to align with the upward electric field

(c) Piezoelectric material poled in the upward direction

(d) Actuation of the piezoelectric material in response to the electric field

The piezoelectric phenomenon is characterized by the relationship between material strain and local charge displacement. The direct piezoelectric effect is the development of 
an electric field in response to an applied stress. The magnitude of the charge displacement is proportional to the magnitude of the applied stress and is expressed in [27] as:

$$
\mathbf{D}=\mathbf{d T}
$$

Where $D$ is the electric displacement per unit volume and $T$ is the stress. $d$ is the piezoelectric coupling coefficient relating stress to electric displacement. Equation 1.1 can be rewritten in matrix form as [27]:

$$
\left[\begin{array}{l}
D_{1} \\
D_{2} \\
D_{3}
\end{array}\right]=\left[\begin{array}{llllll}
d_{11} & d_{12} & d_{13} & d_{14} & d_{15} & d_{16} \\
d_{21} & d_{22} & d_{23} & d_{24} & d_{25} & d_{26} \\
d_{31} & d_{32} & d_{33} & d_{34} & d_{35} & d_{36}
\end{array}\right]\left[\begin{array}{l}
T_{1} \\
T_{2} \\
T_{3} \\
T_{4} \\
T_{5} \\
T_{6}
\end{array}\right]
$$

Where, 1, 2,3 are the Cartesian coordinate system $x, y, z . T_{1}, T_{2}$ and $T_{3}$ represent normal stresses and $T_{4}, T_{5}$ and $T_{6}$ represent shear stresses. It is common to align the 3 direction with the poling direction of the piezoelectric material.

The form of the piezoelectric coupling matrix, $d_{i j}$, will depend on the point group of the crystal structure of the material in question. For example, Lead Zirconate Titanate (PZT), a popular piezoelectric material with a tetragonal crystal structure with a $4 \mathrm{~mm}$ point group, has a piezoelectric coefficient matrix of the form [27]:

$$
\left[\begin{array}{cccccc}
0 & 0 & 0 & 0 & d_{15} & 0 \\
0 & 0 & 0 & d_{15} & 0 & 0 \\
d_{31} & d_{31} & d_{33} & 0 & 0 & 0
\end{array}\right]
$$


The electric displacement, $D$, can also result from an externally applied electric field. The magnitude of the electric displacement is proportional to the electric field, $E$, and the permittivity of the material, $\varepsilon$. This relationship is expressed in Equation 1.4 [26].

$$
\mathbf{D}=\boldsymbol{\varepsilon} \overline{\mathbf{E}}
$$

The electric displacement also can be a result of both the electric field and applied stresses. Electric displacement can then be re-expressed as [26]:

$$
\mathbf{D}=\mathbf{d} \mathbf{T}+\boldsymbol{\varepsilon} \overline{\mathbf{E}}
$$

The converse piezoelectric effect is the phenomenon characterized by the deformation of a material in response to an applied electric field. The strain in the material, $S$, is proportional to the electric field throughout the material, $E$, and the piezoelectric coupling of the material, $d$. Strains in the material can also occur as a result of applied mechanical stress. The magnitude of the strains in the material, $S$, are proportional to the magnitude of the applied stresses, $T$, and the compliance of the material, $s$. Strains of the material resulting from applied electric field and mechanical stress is expressed in Equation (1.6)

$$
\begin{aligned}
& \mathbf{S}=\mathbf{s} \mathbf{T}+\mathbf{d} \overline{\mathrm{E}} \\
& {\left[\begin{array}{l}
S_{1} \\
S_{2} \\
S_{3} \\
S_{4} \\
S_{5} \\
S_{6}
\end{array}\right]=\left[\begin{array}{llllll}
s_{11} & s_{12} & s_{13} & s_{14} & s_{15} & s_{16} \\
s_{21} & s_{22} & s_{23} & s_{24} & s_{25} & s_{26} \\
s_{31} & s_{32} & s_{33} & s_{34} & s_{35} & s_{36} \\
s_{41} & s_{42} & s_{43} & s_{44} & s_{45} & s_{46} \\
s_{51} & s_{52} & s_{53} & s_{54} & s_{55} & s_{56} \\
s_{61} & s_{62} & s_{63} & s_{64} & s_{65} & s_{66}
\end{array}\right]\left[\begin{array}{l}
T_{1} \\
T_{2} \\
T_{3} \\
T_{4} \\
T_{5} \\
T_{6}
\end{array}\right]+\left[\begin{array}{lll}
d_{11} & d_{21} & d_{31} \\
d_{12} & d_{22} & d_{32} \\
d_{13} & d_{23} & d_{33} \\
d_{14} & d_{24} & d_{34} \\
d_{15} & d_{25} & d_{35} \\
d_{16} & d_{26} & d_{36}
\end{array}\right]\left[\begin{array}{l}
\bar{E}_{1} \\
\bar{E}_{2} \\
\bar{E}_{3}
\end{array}\right] }
\end{aligned}
$$


The converse piezoelectric formulation is most useful for describing the motion of piezoelectric actuators. When considering a PZT-5A (a very common piezoelectric material), Equation (1.6) can be reduced to:

$$
\begin{aligned}
& \mathbf{S}=\mathbf{s} \mathbf{T}+\mathbf{d} \overline{\mathbf{E}} \\
& {\left[\begin{array}{l}
S_{1} \\
S_{2} \\
S_{3} \\
S_{4} \\
S_{5} \\
S_{6}
\end{array}\right]=\left[\begin{array}{cccccc}
s_{11} & s_{12} & s_{13} & 0 & 0 & 0 \\
s_{21} & s_{22} & s_{23} & 0 & 0 & 0 \\
s_{31} & s_{32} & s_{33} & 0 & 0 & 0 \\
0 & 0 & 0 & s_{44} & 0 & 0 \\
0 & 0 & 0 & 0 & s_{55} & 0 \\
0 & 0 & 0 & 0 & 0 & s_{66}
\end{array}\right]\left[\begin{array}{c}
T_{1} \\
T_{2} \\
T_{3} \\
T_{4} \\
T_{5} \\
T_{6}
\end{array}\right]+\left[\begin{array}{ccc}
0 & 0 & d_{31} \\
0 & 0 & d_{32} \\
0 & 0 & d_{33} \\
0 & d_{24} & 0 \\
d_{15} & 0 & 0 \\
0 & 0 & 0
\end{array}\right]\left[\begin{array}{c}
\bar{E}_{1} \\
\bar{E}_{2} \\
\bar{E}_{3}
\end{array}\right] }
\end{aligned}
$$

The system of equations relating the electric displacement, $D$, with the material strains, $S$, is referred to as the Strain-Charge equation form.

These systems of equations can be presented in more desirable forms that include variables of interest. For example, in Finite Element Analysis, it is frequently more convenient to establish boundary conditions or return results in terms of local voltage or displacement. The method for converting the equation forms in order to include the desired variables is presented in Figure 1-5: 


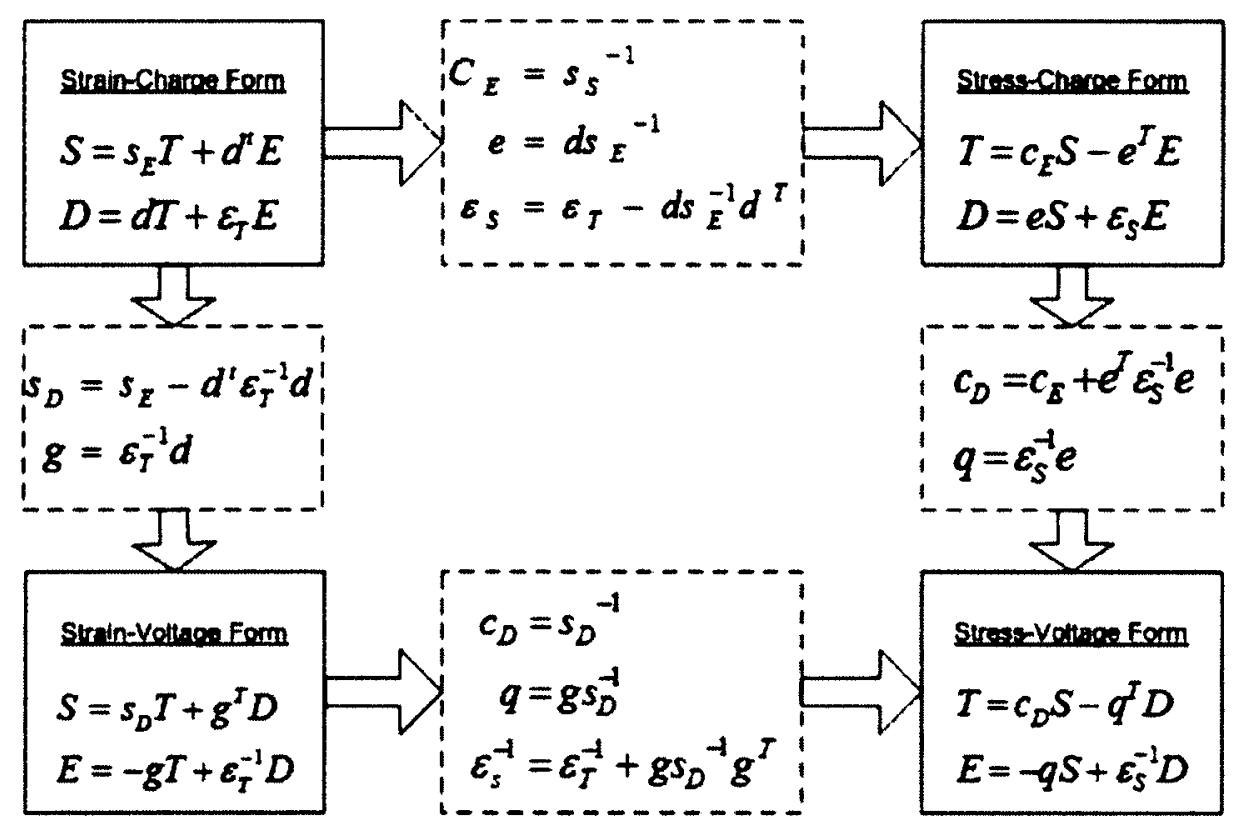

Figure 1-4: Constitutive Equation Forms [28]

Examples of material properties for Lead Zirconate Titanate (PZT-4) are listed in Table 1-1.

Table 1-1: Material and piezoelectric properties of PZT-4 [26]

\begin{tabular}{ll}
\hline Material Properties & PZT \\
\hline Piezoelectric constants & \\
$D_{33}\left(10^{-12} \mathrm{C} / \mathrm{N}\right.$ or $\left.\mathrm{m} / \mathrm{N}\right)$ & 300 \\
$\mathrm{D}_{31}\left(10^{-12} \mathrm{C} / \mathrm{N}\right.$ or $\left.\mathrm{m} / \mathrm{N}\right)$ & -150 \\
$\mathrm{D}_{32}\left(10^{-12} \mathrm{C} / \mathrm{N}\right.$ or $\left.\mathrm{m} / \mathrm{N}\right)$ & -150 \\
\hline Relative Permittivity, $\varepsilon / \varepsilon_{0}$ & 1800 \\
$\left(\varepsilon_{0}=8.85410^{-12} \mathrm{~F} / \mathrm{m}\right)$ & \\
\hline Young's Modulus $(\mathrm{GPa})$ & 50 \\
$\mathrm{E}_{1}$ & 50 \\
$\mathrm{E}_{2}$ & 50 \\
$\mathrm{E}_{3}$ & \\
\hline Maximum stress in traction $(\mathrm{MPa})$ & 80 \\
Direction 1 & 80 \\
Direction 2 & 1 \\
\hline Max electric field $\left(10^{6} \mathrm{~V} / \mathrm{m}\right)$ & 7600 \\
\hline Density $\left(\mathrm{kg} / \mathrm{m}^{3}\right)$ & \\
\hline
\end{tabular}




\subsection{Piezoelectric Actuators}

Piezoelectric actuators are actuators that hamess the piezoelectric effect of some materials in order to provide actuation. Piezoelectric actuators consist of piezoelectric material structures furnished with an array of electrodes that supply the actuating electric potential.

Many types of piezoelectric actuators have been designed using different arrangements of piezoelectric materials and electrodes. The following are examples of piezoelectric actuators to be discussed:

- Piezoelectric plate actuator

- Piezoelectric stack actuator

- Interdigitated electrode monolithic actuator

- Active Fibre Composite (AFC) actuator

- Hollow Active Fibre Composite (HAFC) actuator

- Unimorphic and Bimorphic actuators

Some other piezoelectric actuator configurations are:

- Piezo-hydraulic Pump

- Metal Tube Motor

- Feed-Screw Piezoelectric actuator 


\subsubsection{Piezoelectric Plate Actuator}

The piezoelectric plate actuator consists of a monolithic plate of piezoelectric material. An electric field is applied through the plate. The plate experiences a piezoelectric strain proportional to the intensity of the electric field, $E$, and the magnitude of piezoelectric coupling coefficient, $d$. Figure 1-5 illustrates the piezoelectric plate actuator.

The intensity of the electric field is inversely proportional to the thickness of the plate that separates the voltage sources. The strain induced in the plate can then be increased by decreasing the plate thickness.

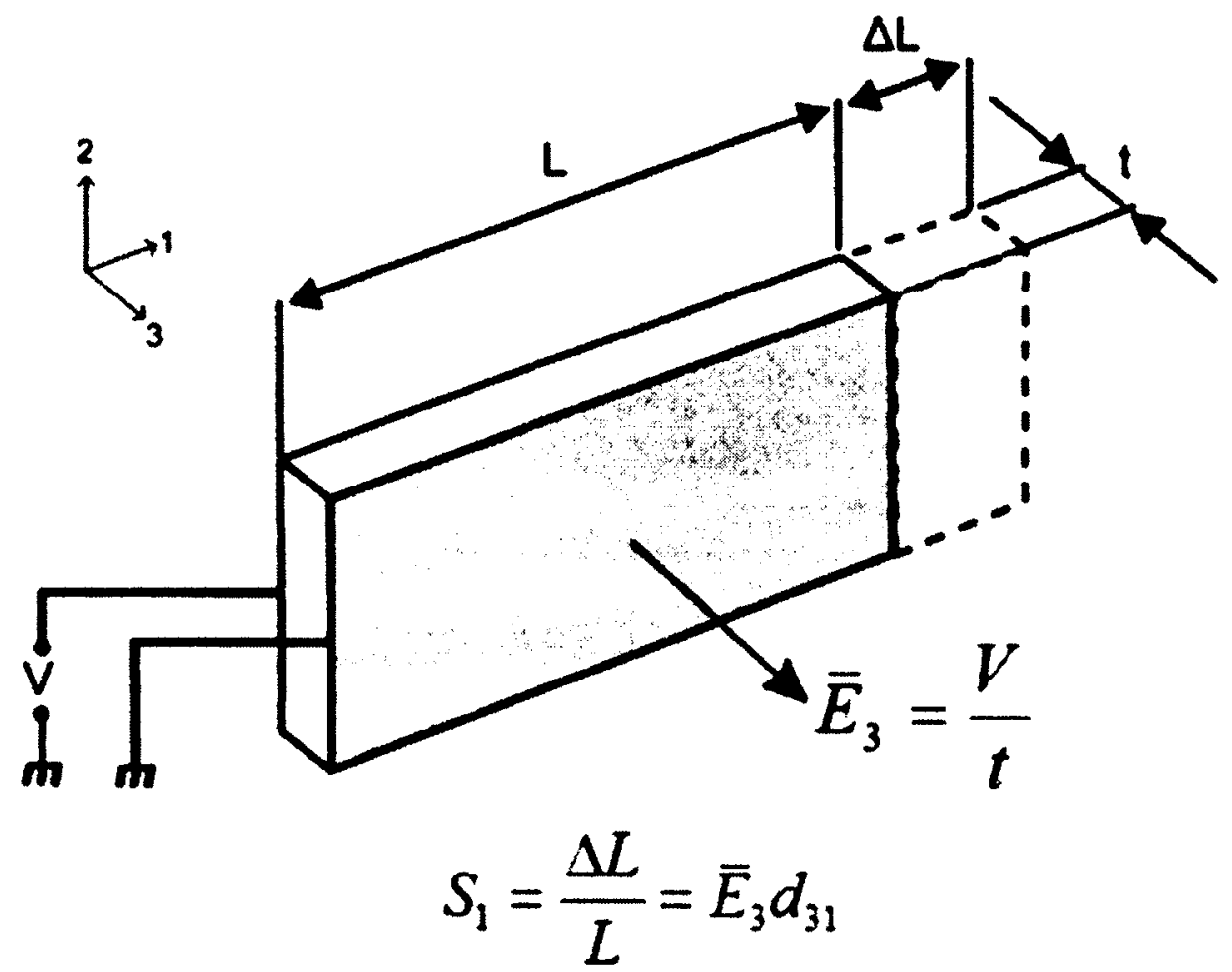

Figure 1-5: Piezoelectric plate actuator [29] 
The actuation strains produced in the piezoelectric plate are outlined in Equation 1.6. When only electric fields and no external stresses are applied, equation 1.6 reduces to equation 1.8 [29]:

$$
S_{1}=d_{31} \bar{E}_{3}+s T=d_{31} \bar{E}_{3}+s(0)=d_{31} \bar{E}_{3}
$$

Strains produced in the material are proportional to the orthogonal piezoelectric coupling coefficient, $d_{3 l}$. The $d_{3 l}$ piezoelectric coupling coefficient is not the most beneficial coefficient to use. $d_{33}$ is larger. Different configurations of piezoelectric actuators have been formulated to take advantage of other, larger piezoelectric coupling coefficients. Such configurations include piezoelectric stack actuators and the interdigitated electrode-based actuators.

\subsubsection{Piezoelectric Stack Actuator}

The piezoelectric stack actuator is designed to take advantage of the $d_{33}$ piezoelectric coupling coefficient.

The piezoelectric stack actuator consists of plates of piezoelectric material separated by sheets of conductive material. A potential difference is created between the alternating conductive sheets creating local electric fields between the conductive sheets. The local electric field direction alternates between adjacent electrodes. Likewise, the poling direction of the piezoelectric material alternates between adjacent electrodes. The electric fields actuate the piezoelectric material located between the conductive sheets. The piezoelectric plate material is poled in the same direction as the local electric field. The 
result is a strain through the thickness of the piezoelectric material. The piezoelectric stack actuator is illustrated in Figure 1-6.

The actuation strains, $S_{3}$ produced in the piezoelectric stack are a function of the electric field, E3 and the piezoelectric coupling coefficient. The relationship is outlined in Equation 1.6 [27]. With some rearrangement, Equation 1.6 reduces to:

$$
S_{3}=n d_{33} \bar{E}_{3}
$$

Since the parallel piezoelectric coupling coefficient $d_{33}$ is typically much larger than the orthogonal piezoelectric coupling coefficient $d_{31}$ (used in the piezoelectric plate actuator), the actuator that takes advantage of this larger coefficient will see a greater actuation performance.

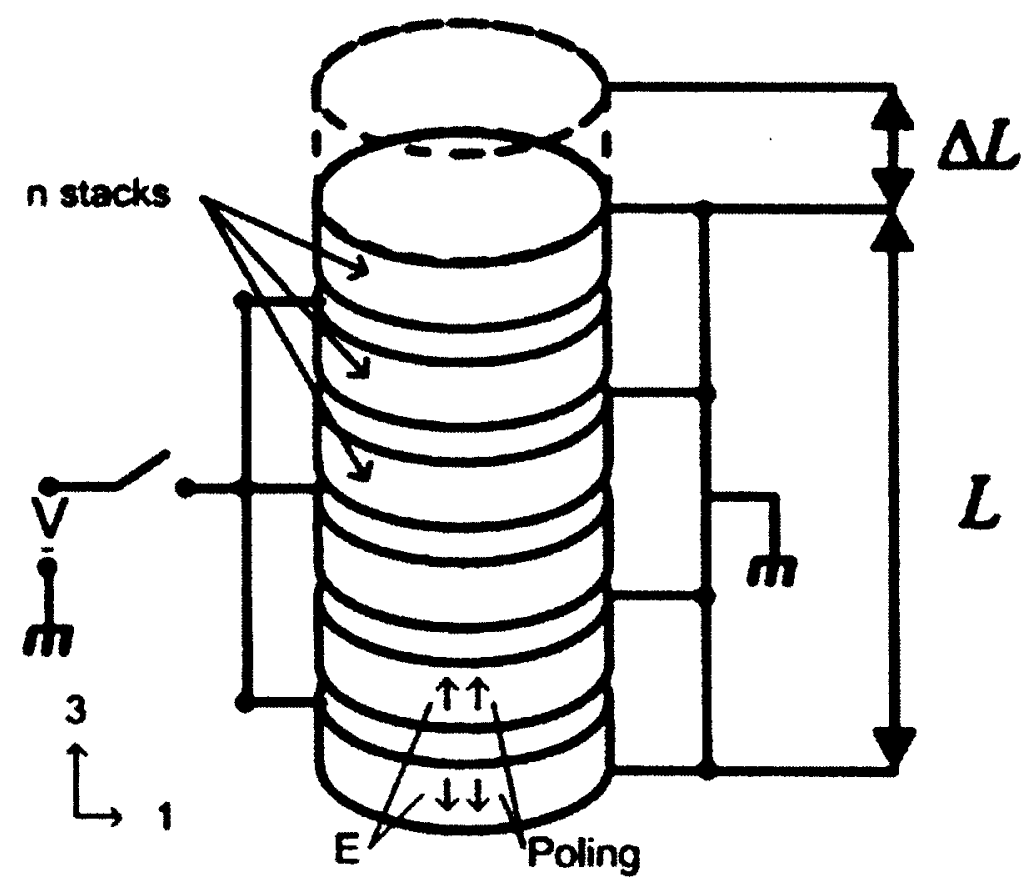

Figure 1-6: Piezoelectric stack actuator [29] 


\subsubsection{Interdigitated Electrode Monolithic Actuator}

The interdigitated electrode monolithic actuator is another configuration designed to take advantage of the $d_{33}$ piezoelectric coupling coefficient.

The interdigitated electrode monolithic actuator consists of a monolithic plate of piezoelectric material sandwiched between two arrays of interdigitated electrodes (IDE) [30]. A potential difference is created between the alternating electrode fingers. This alternating potential difference generates an electric field. An electric field is generated between these alternating electrodes. The electric field leaves the electrode, turns, flows through the piezoelectric material, turns again and arrives at the next adjacent electrode. The piezoelectric material deforms with exposure to the electric field. The interdigitated electrode monolithic actuator is illustrated in Figure 1-7.

The magnitude of the actuator deformation is proportional to the electric field intensity. The electric field intensity is proportional to the potential difference between alternating electrodes and is inversely proportional to the electrode spacing. Since the magnitude of actuator deformation is inversely proportional to the electrode spacing, the reduction of the electrode spacing is desirable. However, there is a limitation to the reduction in electrode spacing due to limits in manufacturability as well as the risk of arcing. 


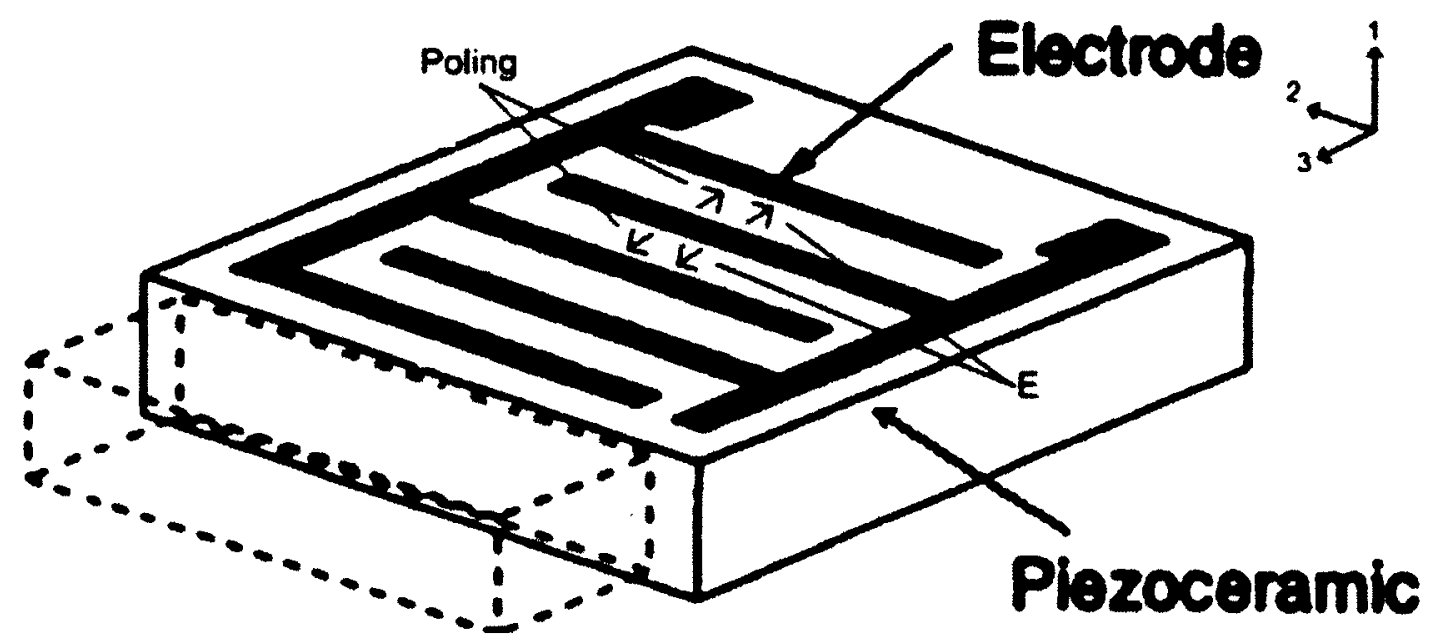

Figure 1-7: Interdigitated electrode monolithic actuator [30]

The actuation strains produced in the monolithic piezoelectric actuator are outlined in Equation 1.10. Equation 1.6 reduces to Equation 1.10 when only electric fields and no external stresses are applied:

$$
S_{3}=d_{33} \bar{E}_{3}
$$

\subsubsection{Active Fibre Composite (AFCs) and Macro Fibre Composites (MFCs)}

Monolithic piezoceramic materials are brittle. In piezoceramic composites, the piezoceramic material are manufactured as fibres, which are then embedded into a matrix material. Piezoceramic composites are more resistant to breakage than their monolithic counterparts.

Piezoelectric fibre composites consist of piezoelectric fibres embedded in a material matrix with a pair of interdigitated electrode buses. An electric potential difference is 
generated between the interdigitated electrodes. The resulting electric field distribution produces a strain in the piezoelectric fibres. The strain in the fibres leads to the deformation of the entire composite structure. The AFC is illustrated in Figure 1-8.

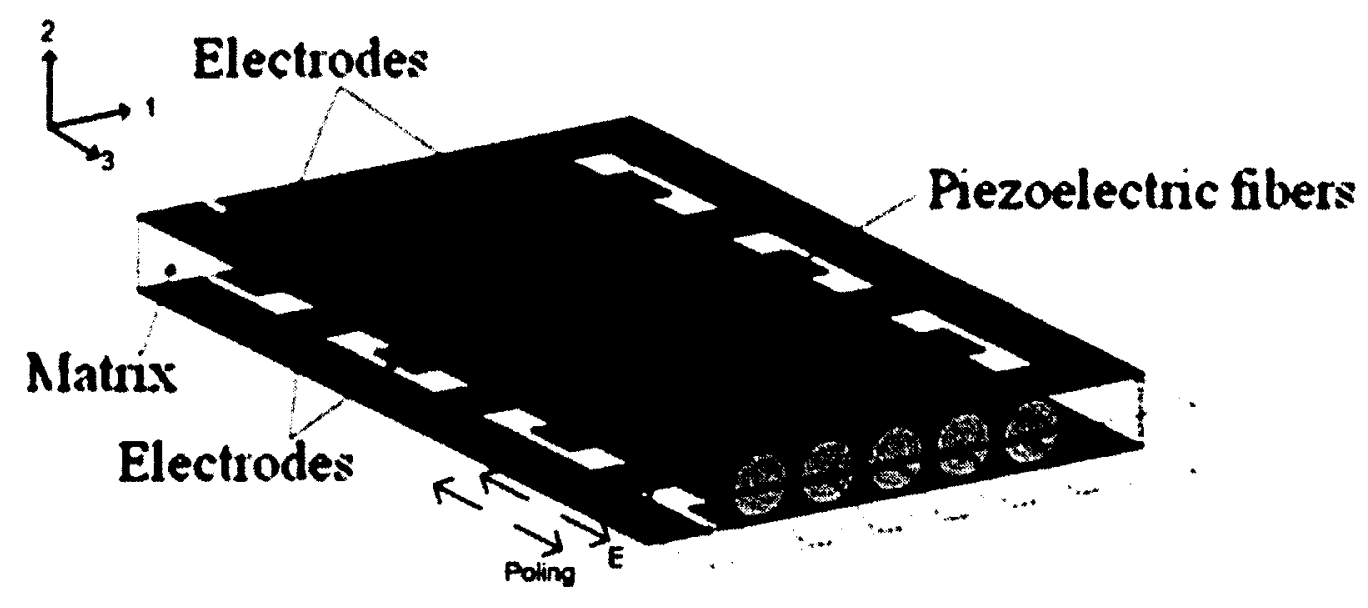

Figure 1-8: Active Fibre Composite (AFC) actuator [31]

There are two classes of Piezoelectric Fibre Composites: Active Fibre Composites (AFCs) and Macro Fibre Composites (MFCs). These classes of AFCs are segregated based on the geometry of the active fibres embedded in the composite. The term Active Fibre Composites (AFCs) refers to composites containing fibres that have a circular cross-section. Piezoelectric fibre composites containing square or rectangular crosssectioned fibres are referred to as Macro Fibre Composites (MFCs).

The magnitude of the actuation in AFC and MFC-based actuators is determined by the magnitude of the electric field flux through the piezoelectric material. The magnitude of the electric field flux through the piezoelectric material is determined by the magnitude of the applied electric potential difference between adjacent electrodes and the 
spacing between these electrodes, the contact area of the electrode to the piezoelectric material and the thickness of the composite [30]. Figure 1-9 illustrates the electric field flux through the active fibres of circular cross-sectioned AFCs and rectangular crosssectioned MFCs. The electrodes conform more closely to the shape of the fibre in the MFC than in the AFC. As such, the electric field flux is slightly higher though the rectangular cross-sectioned fibres of the MFC than through the circular cross-sectioned fibres of the AFC. This higher electric field flux through the fibre results in more significant actuation of the MFC.
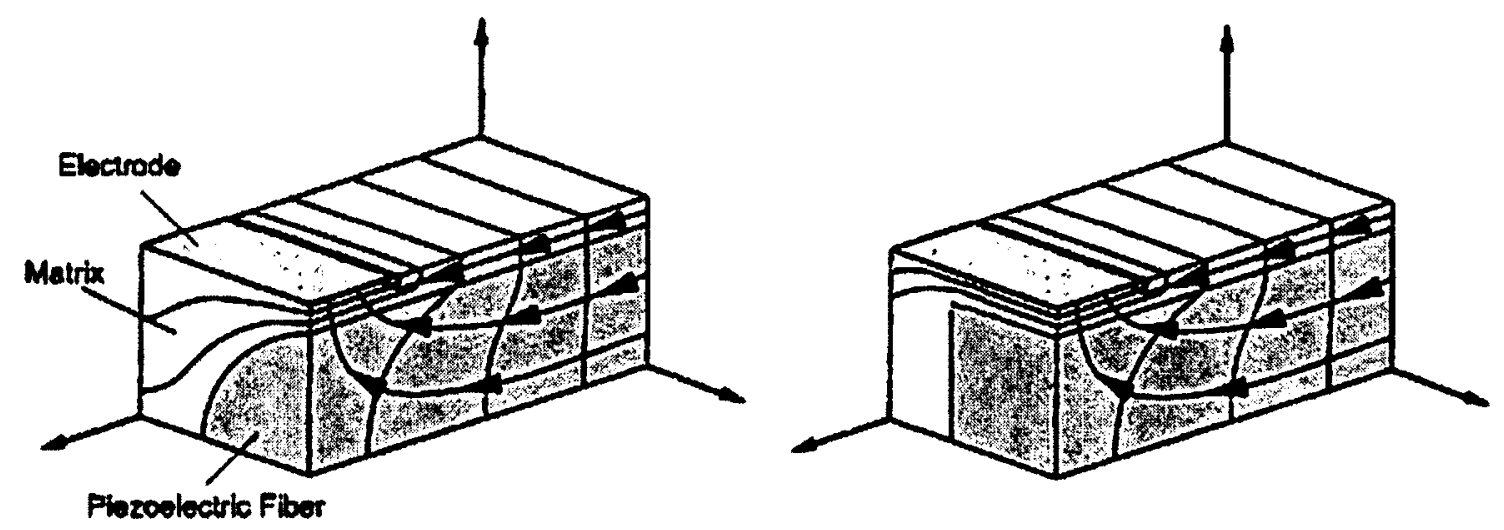

Figure 1-9: Electric field flow through AFC (left) and MFC (right) (adapted from [30])

The circular cross-sectioned fibres of AFCs can be advantageous. Circular fibres can be manufactured using simple extrusion or spinning methods. Also, the ambiguous rotational orientation of circular fibres leads to eased manufacturing of circular fibrebased composites.

The rectangular cross-sectioned fibres of MFCs also offer some advantages. Rectangular fibres can be manufactured using cutting methods. Also, the electrodes of 
MFCs conform more closely to rectangular fibres than to circular fibres resulting in a higher electric field flux, and consequently higher actuation.

Circular cross-sectioned fibres are manufactured using one of two common manufacturing methods: extrusion or Viscous Suspension Spinning Process (VSSP) [32]. Rectangular cross-sectioned fibres are manufactured using one of two manufacturing methods: extrusion or by precision cutting of bulk ceramic.

Both the circular and rectangular fibres can be manufactured via extrusion. Bulk, green material is produced. The material is ground and a feed rod is produced. The feed rod is drawn through a die in the desired cross-section. The fibres of green material are sintered and the fibre is complete. One fibre extrusion process is charted in Figure 1-10.

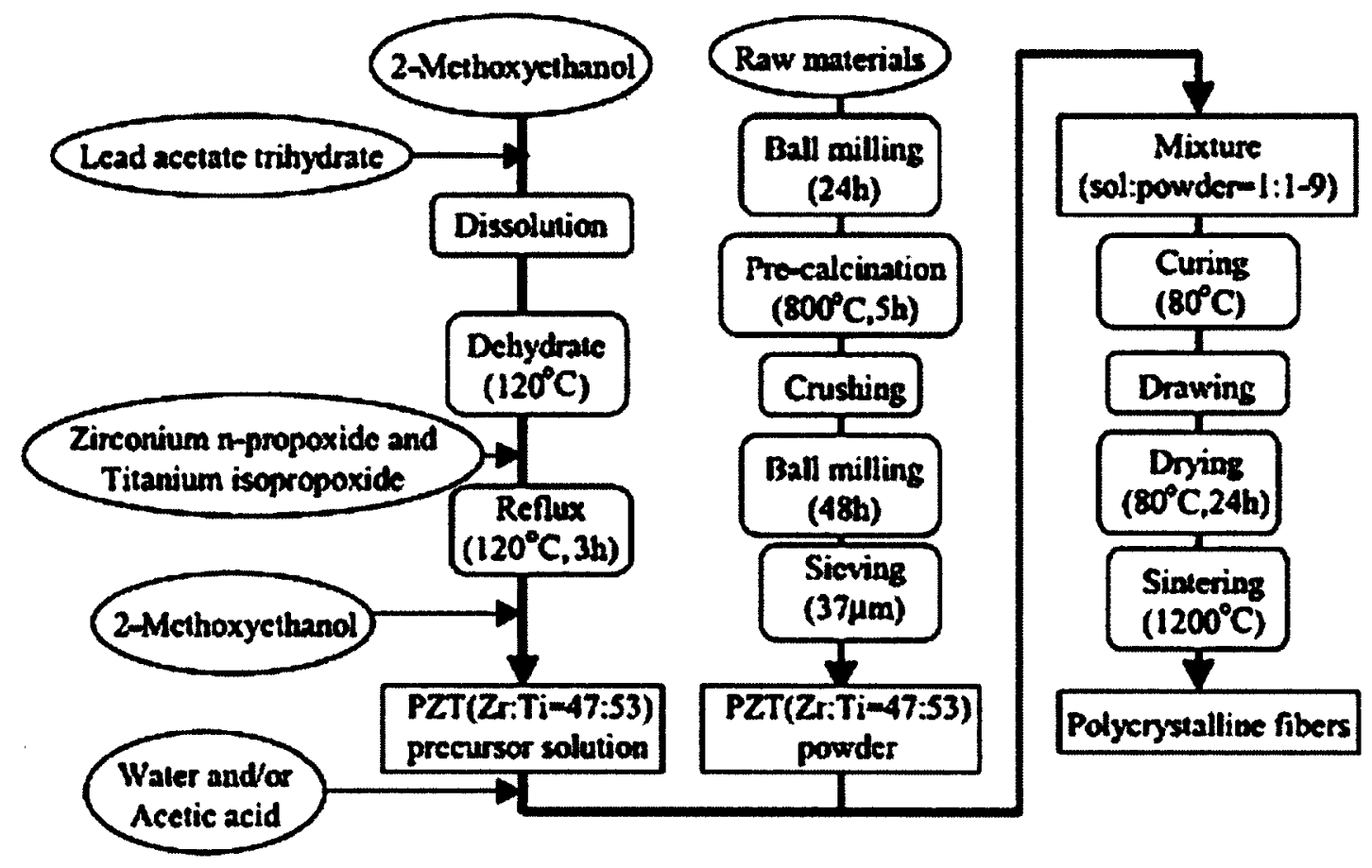

Figure 1-10: Piezoelectric fibre extrusion process 
There is one significant disadvantage to the interdigitated electrode configuration. The interdigitated electrodes create an inhomogeneous electric field path. The inhomogeneous electric field distribution creates an inactive region in the vicinity of the electrodes. Additionally, the inhomogeneous electric field can also cause cracking in the material during poling. [34]. The inhomogeneous electric field path is illustrated in Figure 1-11.

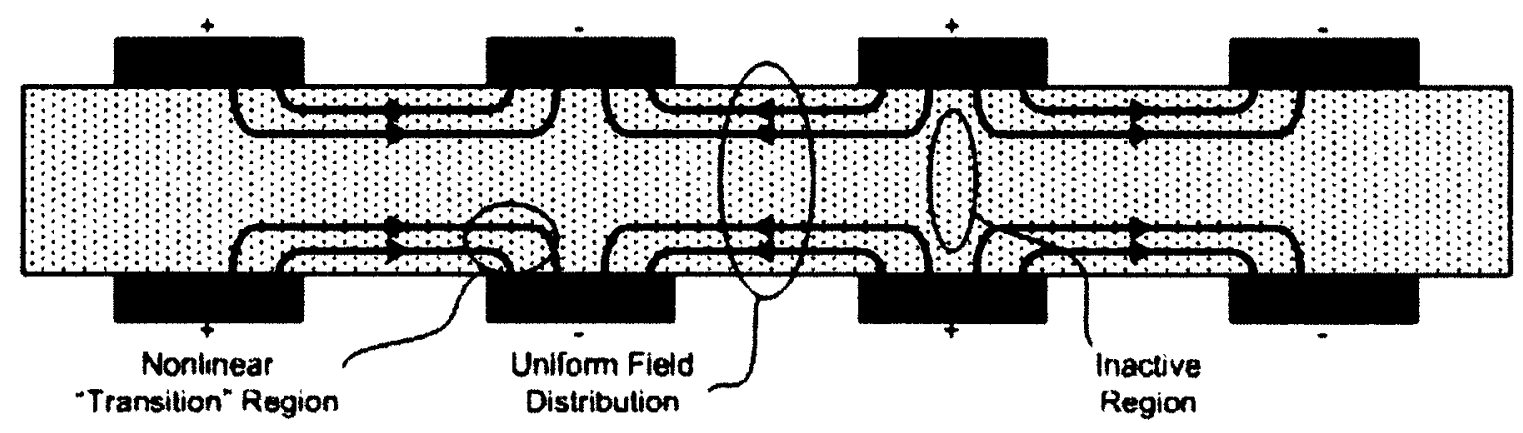

Figure 1-11: Inhomogeneous electric field path [34]

\subsubsection{Hollow Active Fibre Composite Actuator}

Hollow active fibre-based actuators employ a linear, radial electric field path and avoid the disadvantages associated with nonlinear electric field distributions.

The hollow fibre actuator consists of a hollow fibre of piezoelectric material contained in a conductive matrix material that acts as an electrode. A potential difference is created between the inner and outer walls of the fibre. An electric field is generated in the radial direction, between the inner and outer walls of the fibre. The piezoelectric material is poled in the radial direction. The electric field generated between the inner and outer walls induces strain in the piezoelectric material in the longitudinal direction. 
The strain induced in the piezoelectric material is proportional to the intensity of the electric field, $\bar{E}$, and the magnitude of the piezoelectric coupling coefficient, $d_{31}$. The intensity of the electric field, and therefore, the magnitude of the fibre strain, is inversely proportional to the thickness of the fibre wall. It is desirable to minimize the fibre wall thickness. Limitations on manufacturability are what govern the minimum fibre wall thickness. The hollow fibre actuator is illustrated in Figure 1-12.

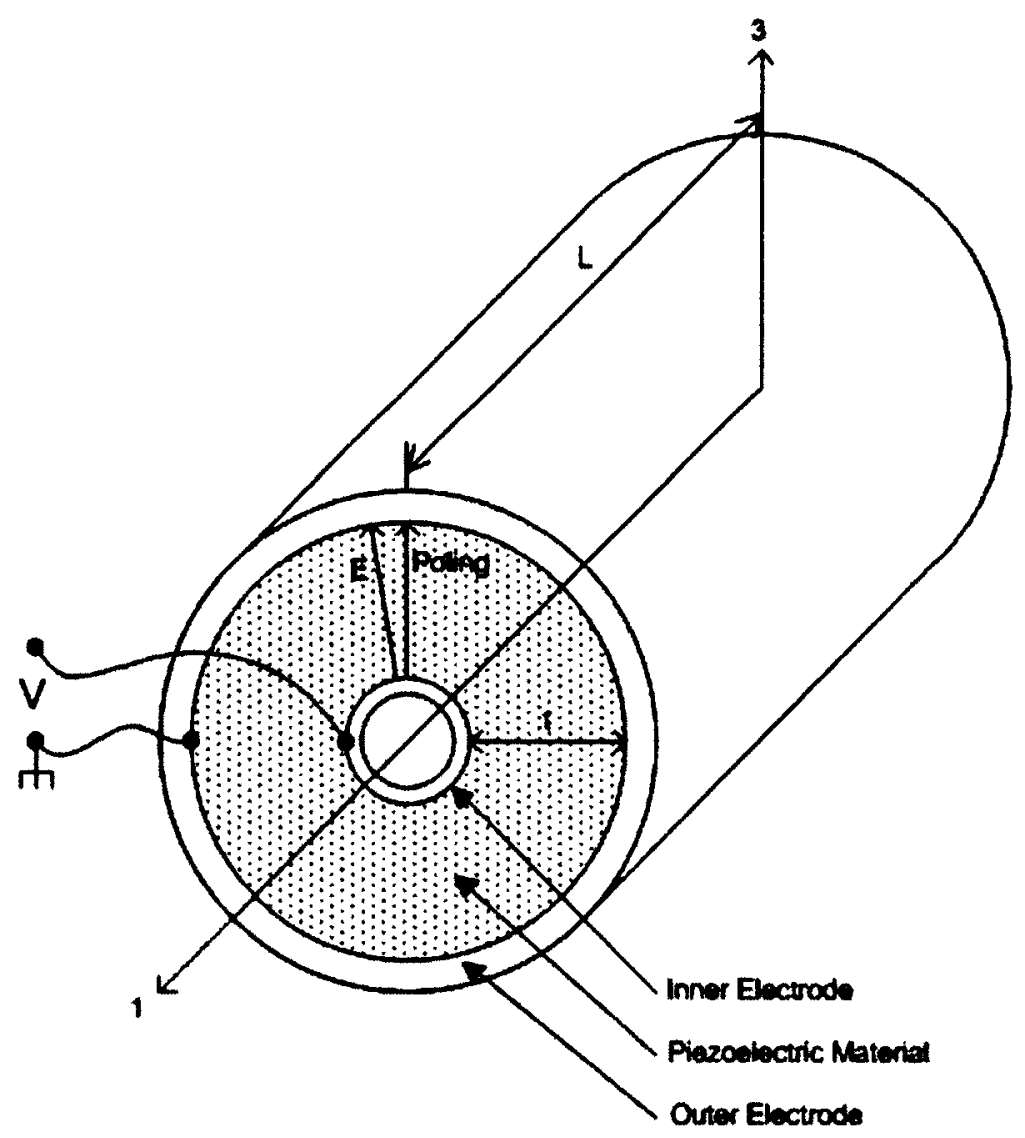

Figure 1-12: Hollow Fibre Actuator [4]

Among piezoceramic-base composite actuators, the hollow piezoelectric fibre-based Hollow Active Fibre Composite (HAFC) offers many advantages over the Active Fibre 
Composite (AFC), including: increased reliability [8], reduced activation potential [35], a higher maximum actuation potential and a broader options for matrix materials [35]. The HAFC is also hindered by some disadvantages not present in AFCs, including: reduced actuation performance and complexity of fibre manufacturing.

The HAFC offers the advantage of increased reliability over AFCs. Since the electrodes are consistently present throughout the entire length of the hollow fibre, cracks in the fibre will not interrupt the electric field path. As such, hollow fibre composite actuator maintains its actuation performance in the event of fibre cracking better than the Active Fibre Composite (AFC) [8].

The HAFC offers the advantage of increased reliability over AFCs. Since the electrodes are consistently present throughout the entire length of the hollow fibre, cracks in the fibre will not interrupt the electric field path. As such, hollow fibre composite actuator maintains its actuation performance in the event of fibre cracking better than the Active Fibre Composite (AFC) [8].

AFCs are constrained to only having non-conductive matrix materials. Conversely, HAFCs can have any matrix material, independent of conductivity and may in fact be advantageous by having a conductive material matrix.

HAFCs can use a higher actuating electric potential than AFCs. AFCs rely on a series of interdigitated electrodes. In interdigitated electrodes, electric potentials are separated by material matrix. The risk of electrostatic discharge through this matrix material limits the actuating potential of AFCs. In HAFCs, electric potentials are separated by piezoelectric material. The risk of electrostatic discharge is significantly lower. Thus, 
HAFCs can use a higher actuating electric potential than AFCs. The actuating electric potential of HAFCs is really only the limit of the material to permit this electric potential.

HAFCs typically have a lower actuation performance than AFCs. AFC actuation is proportional to the parallel piezoelectric coupling coefficient, $d_{33}$. HAFC actuation is proportional to the orthogonal piezoelectric coupling coefficient, $d_{31}$. In most materials, the $d_{33}$ coefficient is significantly higher than the $d_{3 l}$ coefficient. This lower actuation performance of HAFCs can be slightly remedied with the use of higher actuation potentials.

Hollow fibre-based composites are hindered by the added complexity related to the manufacturing of the hollow fibres as compared with the manufacturing of solid crosssection fibres. Solid cross-section fibres can be manufactured by various, simple processes. The manufacturing process of hollow fibres relies on a more complex coextrusion process. The co-extrusion process is illustrated in Figure 1-13. The piezoceramic material is simultaneously extruded about a polymer / carbon material. Precisely manufactured extrusion dies are required to achieve this simultaneous coextrusion. The polymer / carbon material is subsequently removed during the burnout / sintering process, leaving only the hollow piezoceramic fibre. 


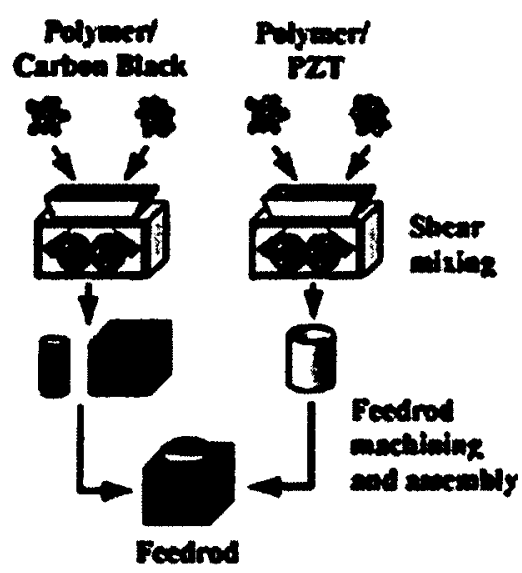

(a)

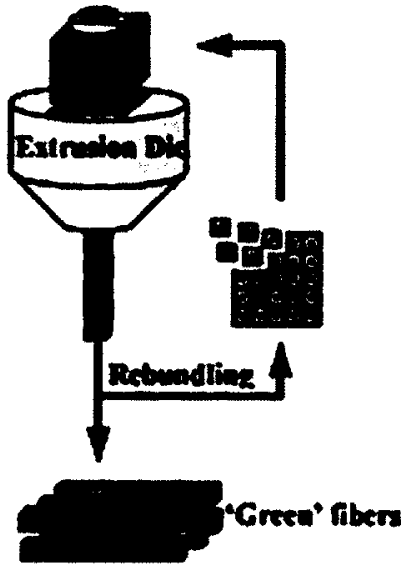

(b)
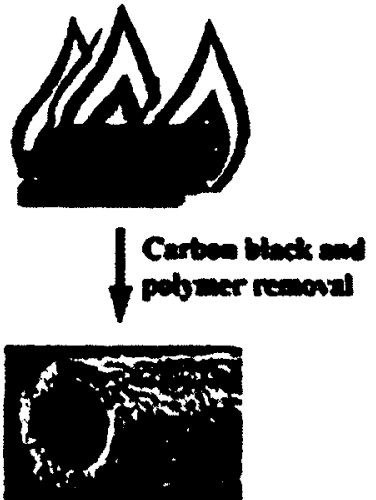

Final mber

(c)

Figure 1-13: Hollow Piezoceramic Fibre Coextrusion Process [36]

(a) Feed rod formation: Carbon / polymer mold for PZT / polymer hollow fibre

(b) Feed rod extrusion: Feed rod is (re)extruded until desired size is produced

(c) Sintering: Carbon and polymer are removed

\subsubsection{Unimorphic and Bimorphic Actuators}

Unimorphic and bimorphic actuators are actuators that undergo bending rather than linear extension, as with the previously discussed actuators. As such, the unimorphic and bimorphic actuators can achieve a larger displacement at the free end than a typical extension-type actuator. Unimorphic and bimorphic structures are illustrated in Figure 114.

Unimorphic structures consist of a piezoelectric actuator bonded to a compliant substrate. Extension of the piezoelectric actuator results in a stress distribution about the substrate and bending or the structure. Bimorphs consist of piezoelectric actuators bonded 
to both sides of a substrate. Antiparallel actuation of the piezoelectric actuators result in more bending than the comparable unimorphic structure.

These bimorphic actuators are of interest in the field of Micro Air vehicles. MAVs can use the bending properties of the bimorphic actuator to serve as an aileron or flap actuator.
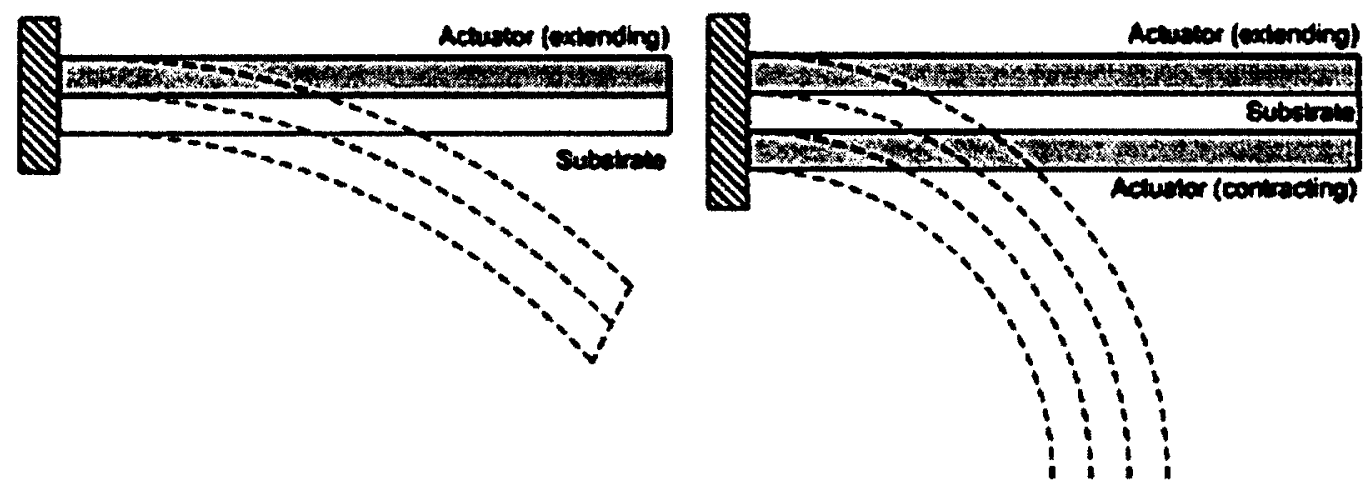

Figure 1-14: Unimorphic (left) and bimorphic (right) structures

\subsubsection{Piezohydraulic Pump}

The piezohydraulic pump is a pump for driving fluid flow [37]. The piezohydraulic pump employs a stack actuator to drive a diaphragm and subsequently move a fluid. The Piezohydraulic pump would be suitable for hydraulic operation. The piezohydraulic pump is illustrated in Figure 1-15. 


\section{Plezohydrauic Pump}

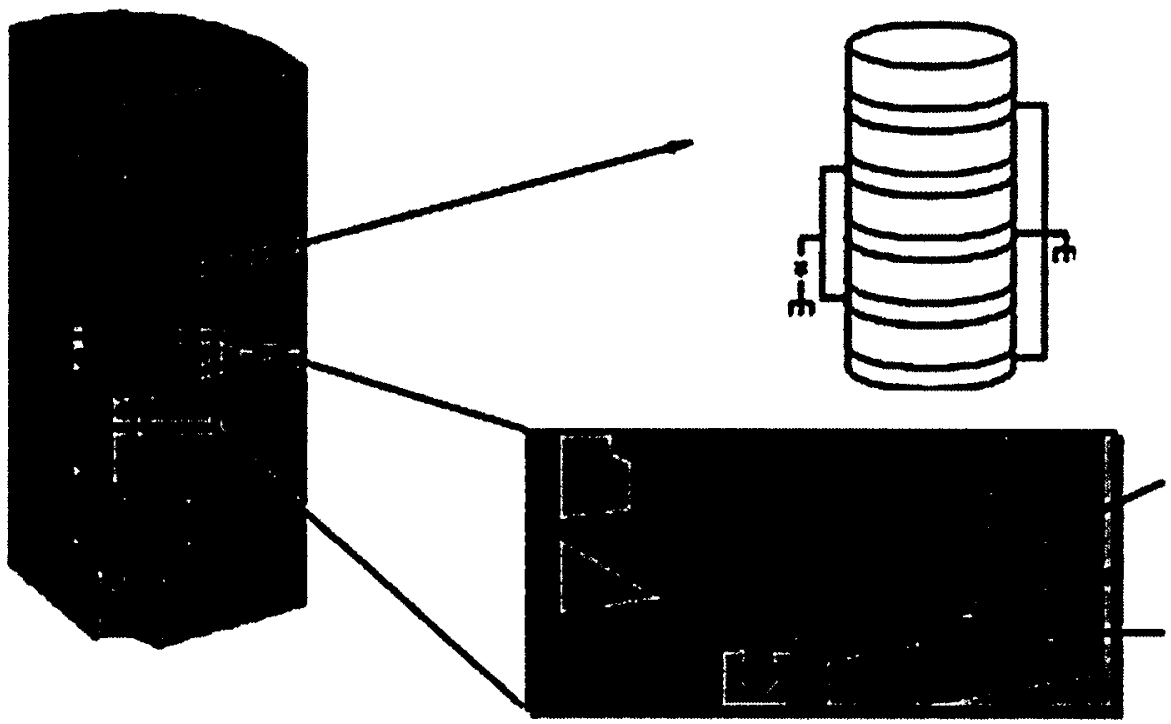

\section{Plezo Stack}

Dlaphragm

Pumplng chamber

Figure 1-15: Piezohydraulic pump [37]

\subsubsection{Metal Tube Motor}

The metal tube motor is a piezoelectric actuator that can provide substantial force at very small scales. The metal tube motor is illustrated in Figure 1-16. Synchronized actuation of the $\mathrm{X}$ and $\mathrm{Y}$ plates produce an elliptical bending motion of the hollow cylinder. This elliptical bending motion can drive a friction-drive rod, producing actuation. The metal tube motor has been used to control the optical zoom function of small cameras. [38] 

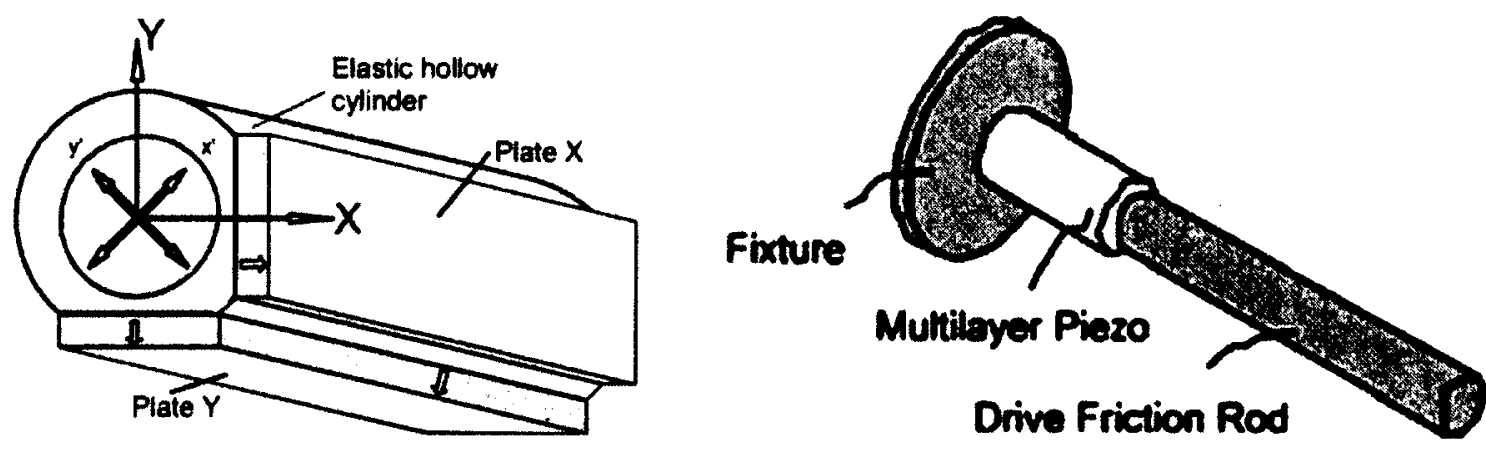

Figure 1-16: Metal tube motor [38]

\subsubsection{Feed-Screw Piezoelectric Actuator}

The Feed-screw piezoelectric actuator consists of a piezoelectric stack actuator mounted to a feed-screw carriage. The result is a linear, motion-accumulating piezoelectric actuator that can provide substantial output power. The feed-screw piezoelectric actuator is illustrated in Figure1-17. The accumulated displacement of the piezoelectric stack would be held in place with an active clamping system. The upper clamp prevents backdriving while the lower clamp allows the stack to advance the load. [39]

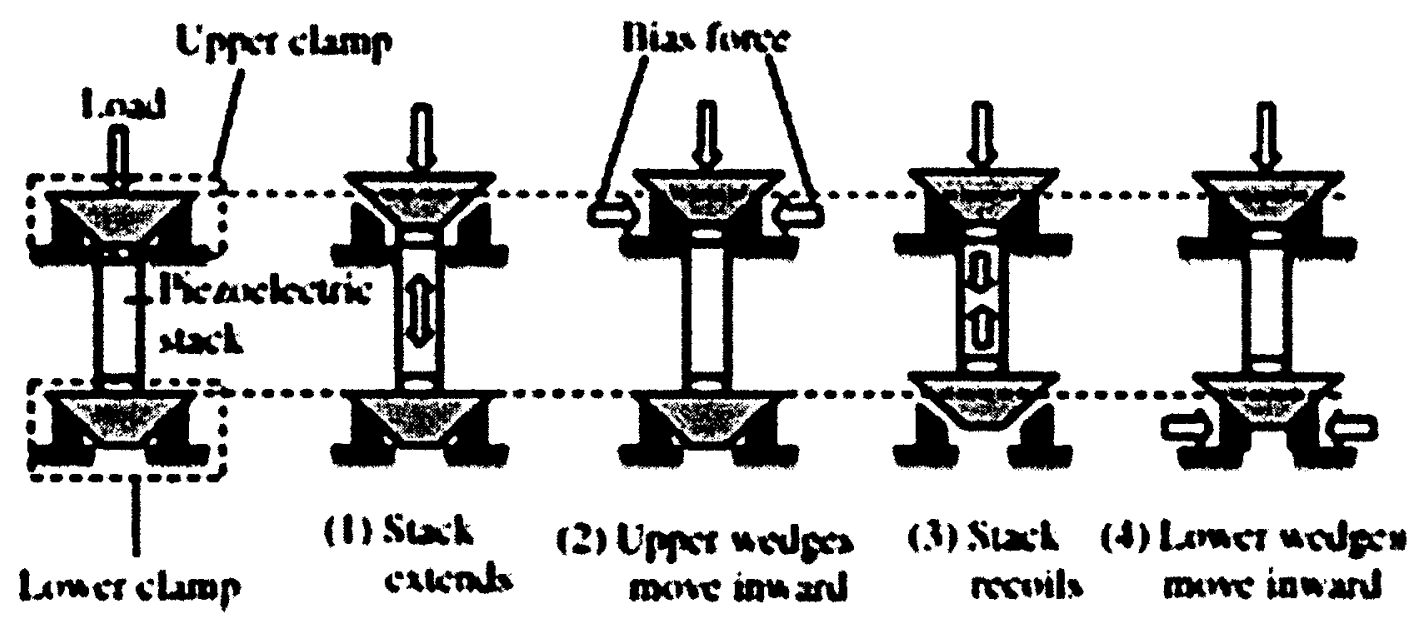

Figure 1-17: Piezoelectric feed-screw actuator [39] 


\subsection{Summary}

The goal of this thesis is to determine the actuation capabilities of bimorphic structures actuated by Active Fibre Composites (AFC) and Hollow Active Fibre Composite (HAFC) based actuators.

AFCs are composite materials containing piezoelectric fibres. The piezoelectric fibres undergo strains when exposed to an electric field. The actuating electric fields in AFCs are currently supplied by interdigitated electrodes. These interdigitated electrodes carry some disadvantages, such as nonlinear electric field distributions. These disadvantages can potentially be avoided with the use of HAFCs.

HAFCs are considered more reliable than AFCs. HAFCs do not employ the problematic interdigitated electrodes used in AFCs. 


\section{Chapter 2: Nonlinear Finite Element Analysis}

\subsection{Introduction}

Structural analyses can be divided into two categories: linear and nonlinear. Nonlinear analyses consider the nonlinear system response. Nonlinear analyses are more difficult to perform than linear analyses however, they are more widely applicable. Many systems can be satisfactorily analyzed while assuming that the response of the system is linear under small loads and /or applied fields. However, nonlinear analysis techniques are required when analyses employing the linear assumption cannot satisfactorily describe the response of the system to large loads and/or applied fields. There are many sources of nonlinearity. Three common sources of nonlinearity are [40]:

1. Material Nonlinearity

2. Geometric Nonlinearity

3. Boundary Condition Nonlinearity

\subsubsection{Material Nonlinearity}

The linear material approximation assumes that a material will always respond in a linear manner until it reaches a critical or failure point. These approximations may be 
satisfactory for the analysis of a system undergoing small deformation or changes in geometry. Nonlinear material properties must be considered if analyses that make use of this approximation give results with insufficient accuracy.

Some examples of material nonlinearity include material critical failure, hardening, softening, or material response hysteresis. These examples are illustrated in Figure 2-1. In Figure 2-1 (a), the material responds linearly until suffering a sudden material failure. In Figure 2-1 (b) the material undergoes hardening, such as in the case of mesh or fibrereinforced materials that undergo mesh or fibre reorientation. In Figure 2-1 (c), the material undergoes softening. In Figure 2-1 (d), the material undergoes plastic deformation. The response curve of a plastically deformed material exhibits a hysteresis. The path of the curve depends on the load history of the material.

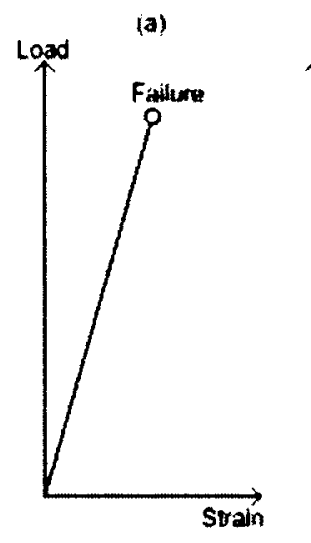

(b)

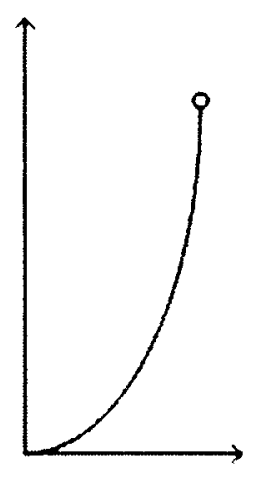

(c)

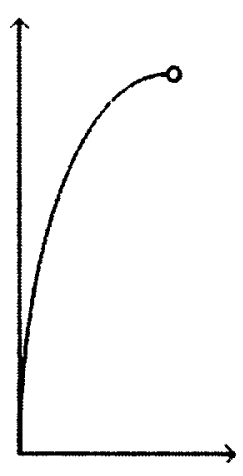

(d)

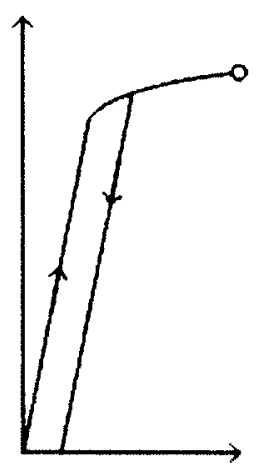

Figure 2-1: Examples of material response [40]

a) Linear until failure

b) Nonlinear Hardening / Stiffening

c) Nonlinear Softening

d) Plasticization 
In addition to mechanical stress, other fields can produce strains in materials. For example, the phenomenon of thermal expansion produces material strain caused by a change in temperature. The piezoelectric phenomenon produces strains in materials in the presence of an electric field.

In addition to having a nonlinear stress-strain relationship, materials can exhibit other nonlinear response relationships with other fields. Figure 2-2 illustrates the nonlinear relationship between electric field and strain in piezoelectric materials. The strain produced in a piezoelectric material is not linear with the applied electric field. Nonlinear analyses of systems containing materials that respond nonlinearly to electric fields may be required. However, nonlinear material effects can be insignificant over small ranges of applied electric field such as in the case of PZT-8 in Figure 2-2.

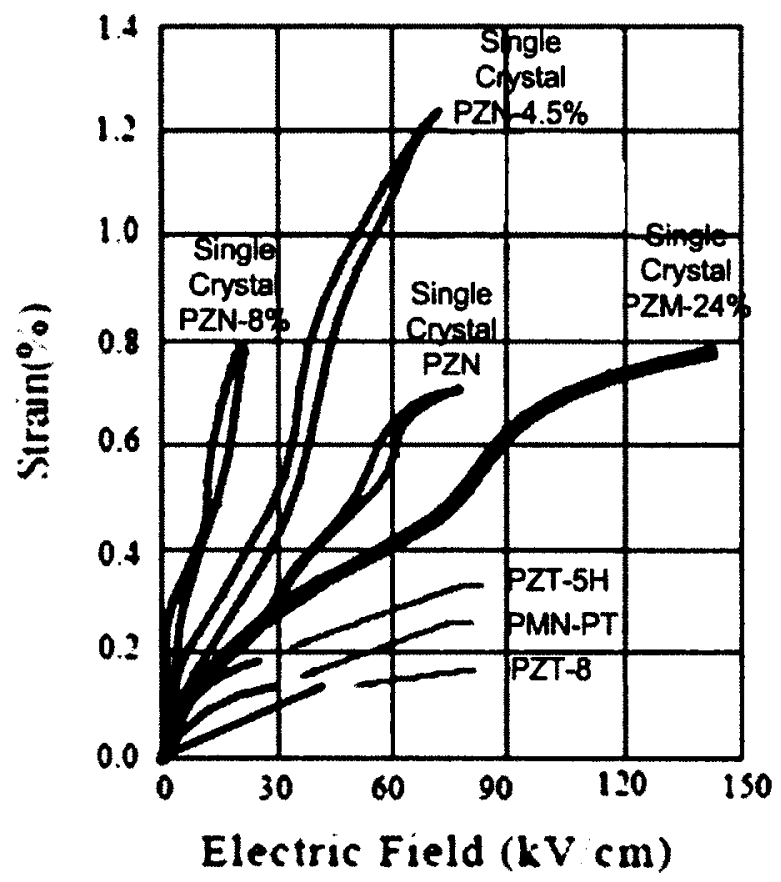

Figure 2-2: Stain vs. electric behaviour for various piezoelectric materials [26] 
Moreover, the piezoelectric response varies with the electric field history and strain history of the material. Such material behaviour is illustrated in Figure 2-3. In Figure 2-3 (a), a small amplitude electric field produces a small, approximately linear strain curve. In Figure 2-3 (b, c, d), larger amplitude electric fields produce larger strain and are subject to larger hysteresis. In Figure 2-3 (e), the electric field amplitude exceeds a critical value, the polarization reorients [26]. The scale of strain that occurs in the smaller amplitude loops $(a, b)$ in Figure 2-3 is of the magnitude where the infinitesimal strain assumption could be used. However, the piezoelectric hysteresis effects are significant and cannot simply be ignored. It may be desirable to accommodate material hysteresis in the nonlinear analysis of a system that contains materials with historydependent properties. 


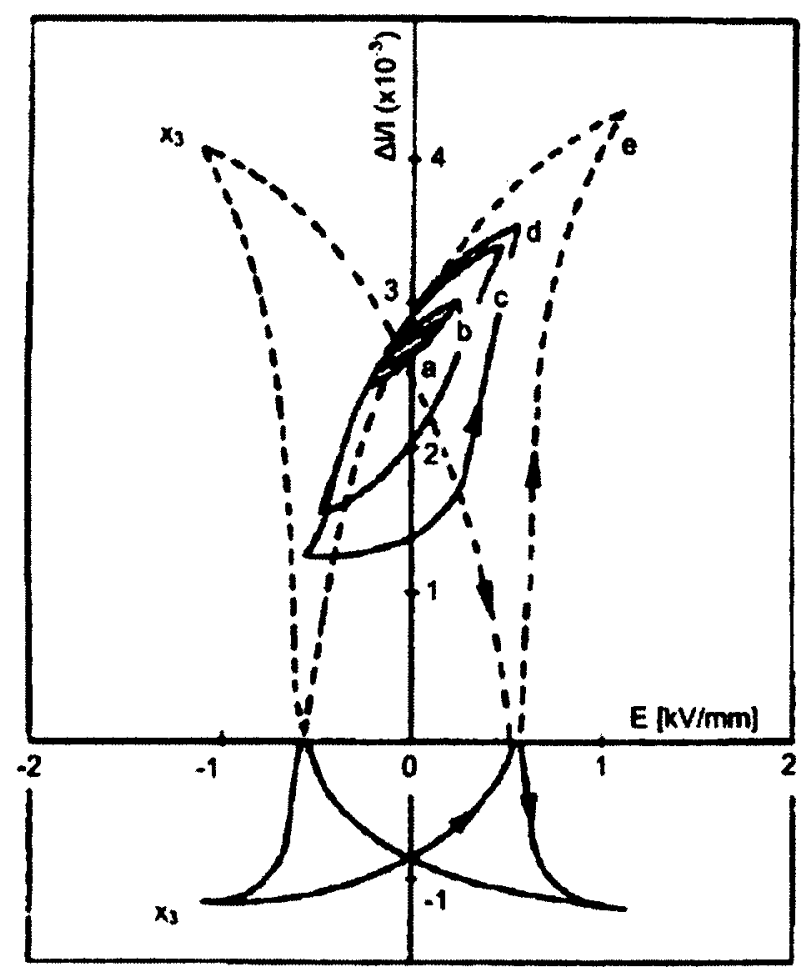

Figure 2-3: Typical strain vs. electric field hysteresis in piezoelectric materials [26]

Material nonlinearity can typically be neglected with the application of the infinitesimal strain assumption. However, it may be necessary to include some nonlinear components of material behaviour in order to obtain sufficiently accurate results.

\subsubsection{Geometric Nonlinearity}

Geometric nonlinear analyses of structures take into account the effect of changes in the stiffness of the structures as the structures deform. Geometric nonlinearity is a symptom of large deformation / rotation of structures.

One example is a member undergoing tensile stress (Figure 2-4). The tensile stress causes a deformation that reduces the material cross-section due to the Poisson's effect 
and consequently changes in the area on which the tensile stress acts. As a result, the stiffness of the structure changes with the applied load.

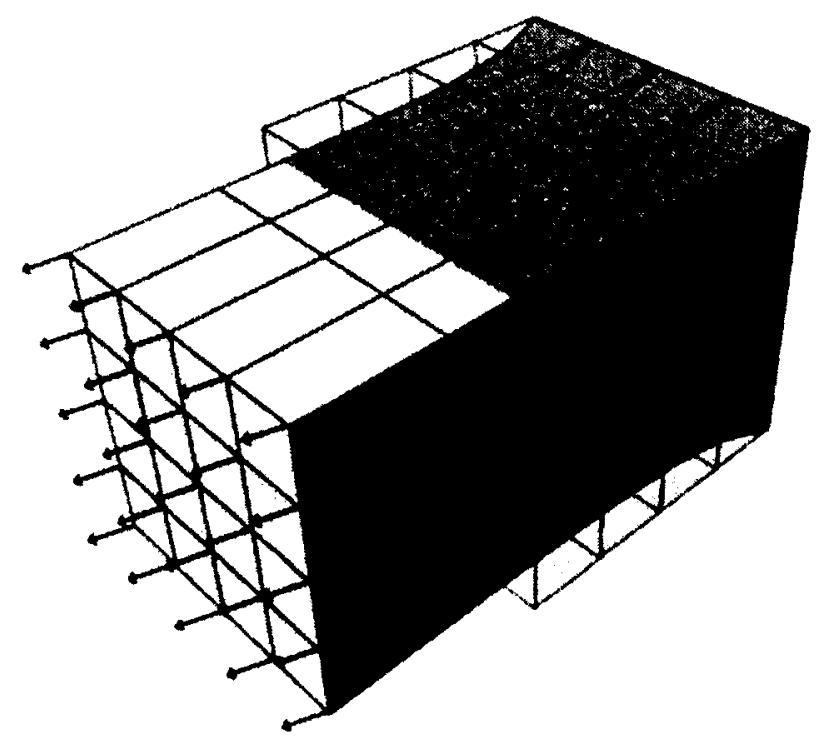

Figure 2-4: Structure subjected to a stress, resulting in large strain deformation

Rotations of the structure can result in changes in relative direction or magnitude of the load. One example is a lateral load on a slender structure. Figure 2-5 illustrates such changes. In Figure 2-5 (a), a slender, cantilevered beam with a cross-sectional area of $A$, a length of $L$, a Young's modulus of $E$ and a second moment of area of $I$ is subjected to a load $P$ at the tip and undergoes a tip displacement $u$. In the initial configuration, the beam responds to the load with relatively little stiffness. The resistance to tip displacement will be approximately $3 E I / L^{3}$. However, as the beam rotates, it will begin to behave as a beam subjected to a longitudinal load and the stiffness of the structure increases. The resistance to tip displacement will approach $E A / L$. These changes in structure stiffness are illustrated in Figure 2-5 (b). 


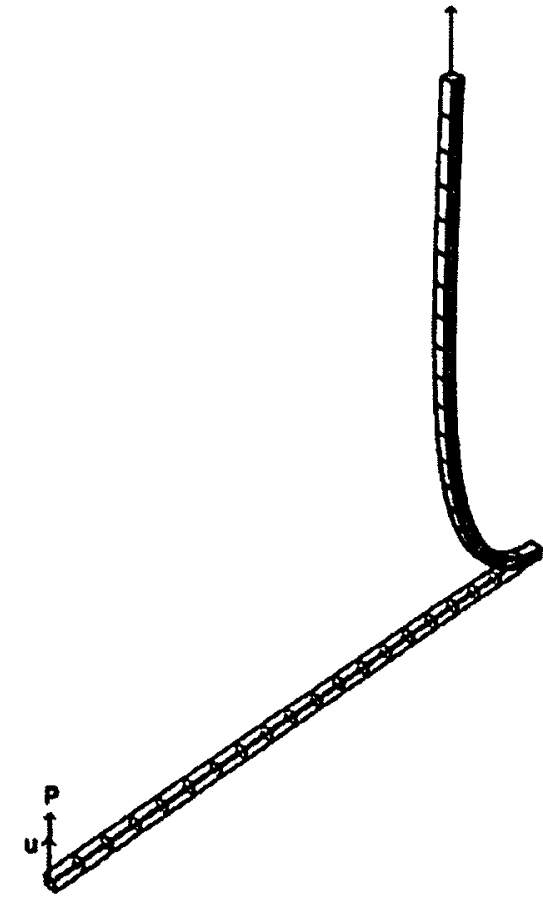

(a)

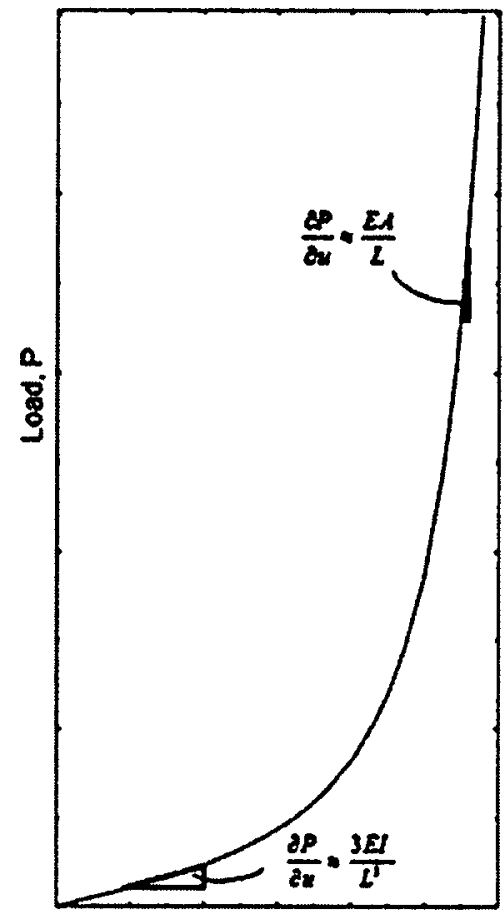

Tip Displecement , $u$

(b)

Figure 2-5: Slender structure subjected to a tip load

a) Slender structure subjected to a tip load

b) Response curve of slender structure subjected to a tip load

In order to explore this example, a finite element analysis was undertaken. A slender beam with the following specifications was created:

$$
\begin{aligned}
& A=4 m^{2} \\
& L=100 \mathrm{~m} \\
& E=100 \times 10^{9} \mathrm{~Pa} \\
& I=1.33 \mathrm{~m}^{4} \\
& (\partial P / \partial u)_{\text {initial }} \approx \frac{3 E I}{L^{3}}=4 \times 10^{5} \frac{\mathrm{N}}{\mathrm{m}} \\
& (\partial P / \partial u)_{\text {final }} \approx \frac{E A}{L}=4 \times 10^{9} \frac{\mathrm{N}}{\mathrm{m}}
\end{aligned}
$$


The finite element model is illustrated in Figure 2-5(a). The beam was assigned a mesh of $20 \times 1 \times 1$ ABAQUS quadrilateral, 20-node C3D20 elements. The beam was fully constrained at one end while a consistently-oriented load was applied at the free end. Tip displacement was computed at the free end.

The beam will have a response curve and corresponding displacement resistance as illustrated in Figure 2-6:

In the initial configuration, the beam responds to the load with relatively little stiffness. The resistance to tip displacement will be $3 E I / L^{3}$ or $4.0 \times 10^{5} \mathrm{~N} / \mathrm{m}$. However, as the beam rotates, it will begin to behave as a beam subjected to a longitudinal load and the stiffness of the structure increases. The resistance to tip displacement will behave asymptotically and approach $E A / L$. 


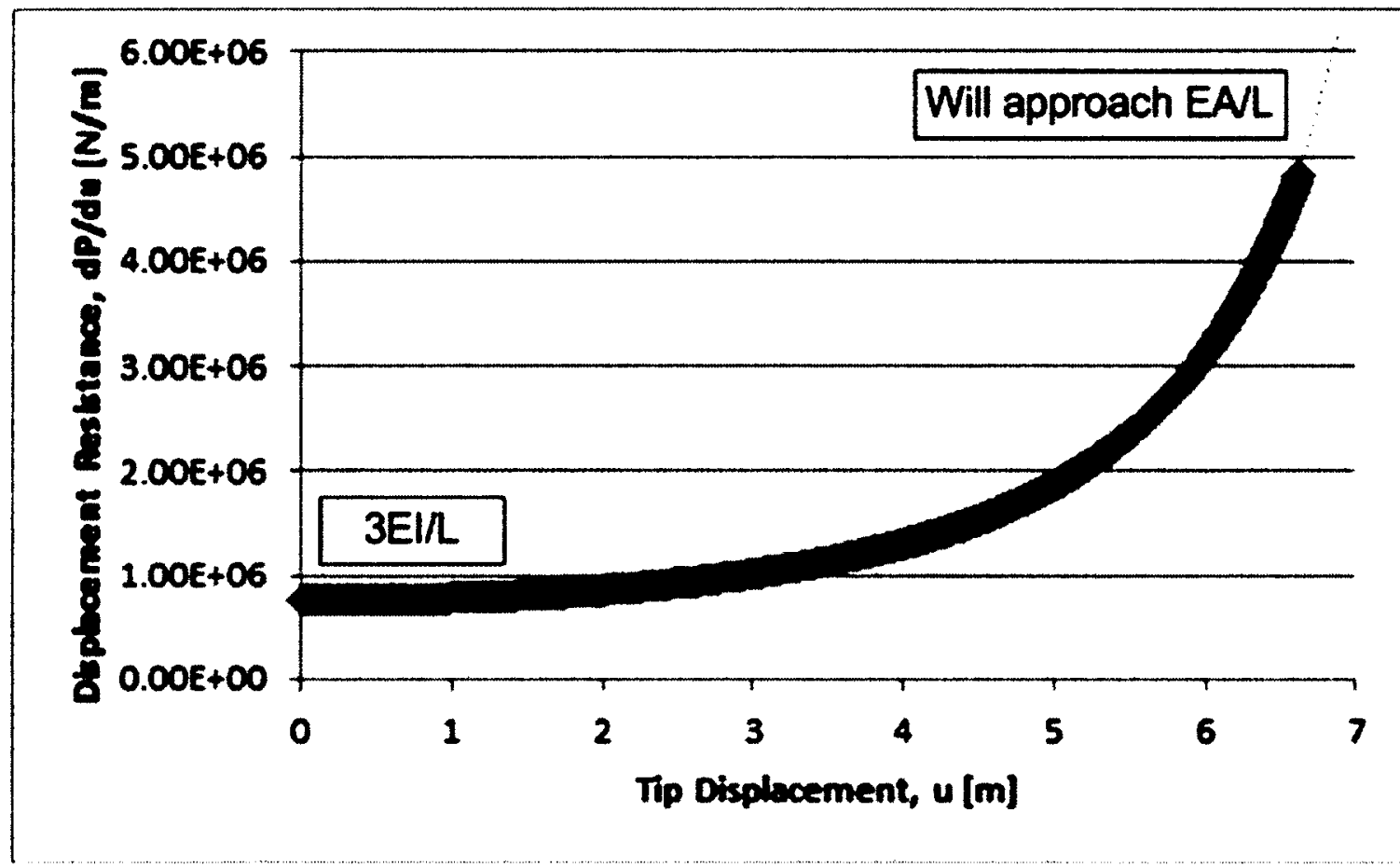

Figure 2-6: Displacement resistance of slender beam subjected to tip load

Load interaction can also be a source of nonlinear behaviour. One example is a nonlinear behaviour resulting from load interaction as illustrated in Figure 2-7. A slender, pin-ended beam is subjected to both longitudinal and transverse loads in Figure 2-7 (a). The linear deformation approximation includes the assumption of deformation superposition. When considered individually, each of the loads produces a relatively modest deformation. When subjected to the axial load alone, as in Figure 2-7 (b), the beam undergoes compression, such that the maximum displacement along the longitudinal direction is $u_{L}=\frac{A P L}{E}$. When subjected to the lateral load alone, as in Figure 2-6 (c), the beam undergoes bending, such that the maximum displacement in the 
transverse direction is $u_{T}=\frac{5 W L^{4}}{384 E I}$. When combined using the deformations superposition assumption, as in Figure 2-7 (d), there is no contribution into transverse displacement from longitudinal load. However, when the nonlinear analysis is considered, as in Figure 2-7 (e), the transverse displacement depends on both the transverse and longitudinal loads. Thus, the maximum displacement obtained in transverse direction is $u_{t}=\frac{w}{P k^{2}}\left(\sec \frac{k L}{2}-1\right)-\frac{w L^{2}}{8 P}$ [42]. The maximum displacement produced by the combined load is far greater than the sum of the displacements caused by the individual loads. 


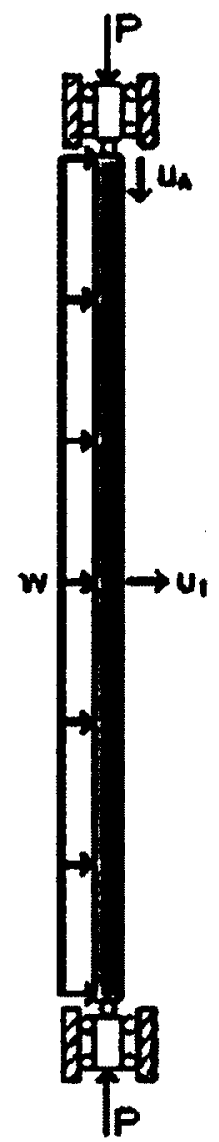

(a)

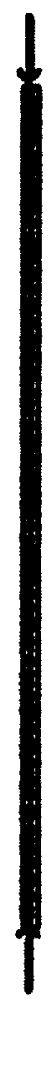

(b)

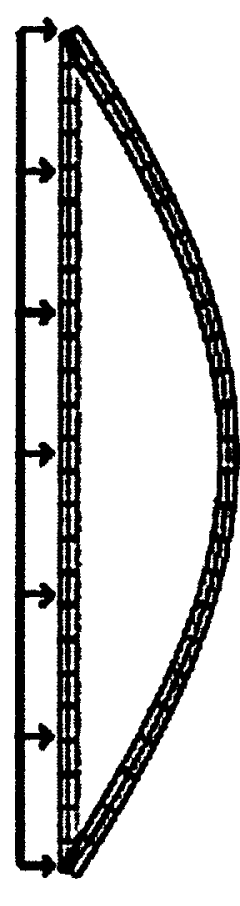

(c)

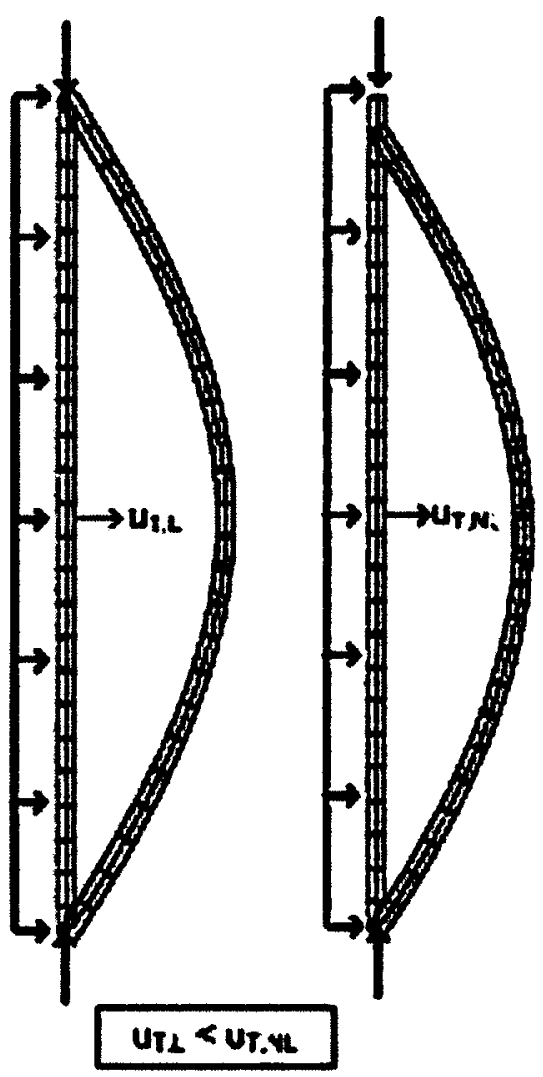

(d)

(e)

Figure 2-7: Pinned beam subjected to axial point loads and lateral pressure load [42]

a) Pinned beam subjected to axial point loads and lateral pressure load

b) Linear analysis of beam subjected to axial loads

c) Linear analysis of beam subjected to lateral pressure load

d) Linear analysis of beam subjected to both axial and lateral loads

e) Nonlinear analysis of beam subjected to both axial and lateral loads 


\subsubsection{Load Nonlinearity or Boundary Condition Nonlinearity}

Finite element analyses must account for boundary condition nonlinearity or load nonlinearity. Boundary condition nonlinearity accounts for changes in boundary conditions with respect to structural deformation. One example of boundary condition nonlinearity is structural interaction. Load nonlinearity accounts for loads that change direction or magnitude with change in structural displacement.

\subsection{Finite Element Analysis (FEA)}

Solving for structures with nonlinear response schemes can be difficult. Solving difficult nonlinear systems can be accomplished with the use of numerical solving methods, such as finite element analysis.

Structural finite element analysis seeks to determine the response of structures to load cases, usually in the form of displacement or strain. Structures are discretized into geometrically simple elements. The response of these simple elements can be determined. The equations determining the response of an element can be assembled into a system of ordinary or partial differential equations in order to determine the response of the structure.

In the case of piezoelectric structural materials, the interrelated system of partial differential equations consist of [8]:

$$
\begin{aligned}
\nabla \mathbf{T}+\mathbf{b} & =0 \\
\mathbf{D} & =0
\end{aligned}
$$


Where $\nabla$ is the divergence operator, $\mathbf{T}$ is the stress and $\mathbf{b}$ are the body forces and $\mathbf{D}$ is the charge displacement. The interrelationship between stresses and charge displacements is described in depth in chapter 1.

The FEA model is defined by the geometry and relevant features of the subject. The model is meshed by discretizing the structure into a series of geometrically simple elements. Elements are selected on the basis of the problem type and degrees of freedom of interest. The discretization process is typically carried out automatically be the finite element analysis pre-processor.

Elemental stiffness matrices are created based on the problem type and degrees of freedom of interest. The stiffness matrices of individual elements are assembled. The assembled elemental stiffness matrices create the stiffness matrix for the model. The loads and boundary conditions of the problem are assigned to the relevant nodes. The loads and boundary conditions, along with the system stiffness matrix create a system of ordinary or partial differential equations. Solving this system of equations devises the response of the system. The response of a system is:

$$
\mathbf{P}=\mathbf{K} \mathbf{u}
$$

Where displacement, $\underline{\boldsymbol{u}}$, or other nodal degree of freedoms are related to load, $\boldsymbol{P}$, or other force function through the elemental stiffness matrix, $\boldsymbol{K}$. The stiffness matrix can be made to accommodate nonlinear analyses with the formulation:

$$
\mathbf{K}=\mathbf{K}_{\mathrm{L}}+\mathbf{K}_{\mathrm{NL}}+\mathbf{K}_{\mathrm{a}}=\overline{\mathbf{K}}+\mathbf{K}_{\mathrm{a}}
$$


$\mathbf{K}_{\mathbf{L}}$ is the infinitesimal strain stiffness matrix, $\mathbf{K}_{\mathrm{NL}}$ is the large deformation stiffness matrix and $\mathbf{K}_{\boldsymbol{\sigma}}$ is the geometric stiffness matrix.

\subsubsection{Linear Stiffness Matrix, $K_{L}$}

The linear stiffness matrix relates load on the element and displacement for the case where the element is undergoing infinitesimal strain. The matrix has the form:

$$
\mathbf{K}_{\mathbf{L}}=\int_{V} \mathbf{B}_{\mathbf{L}}{ }^{T} \mathbf{D B}_{\mathbf{L}} d V=\int_{-1}^{1} \int_{-1}^{1} \int_{-1}^{1} \mathbf{B}_{\mathbf{L}}{ }^{T} \mathbf{D B}_{\mathbf{L}} \operatorname{det}[\mathbf{J}] d \xi d \eta d \zeta
$$

Where $\boldsymbol{D}$ is the material stiffness matrix that relates stress and strain constitutive Equation:

$$
\mathbf{T}=\mathbf{D} \boldsymbol{\varepsilon}
$$

For the simple case of isotropic elastic materials, the $D$ matrix has the form of the material stiffness matrix. The material stiffness matrix establishes the relationship between stress and strain and has the form:

$$
\mathbf{D}=\frac{E}{(1+v)(1+2 v)}\left[\begin{array}{cccccc}
(1-v) & v & v & 0 & 0 & 0 \\
v & (1-v) & v & 0 & 0 & 0 \\
v & v & (1-v) & 0 & 0 & 0 \\
0 & 0 & 0 & \frac{(1+2 v)}{2} & 0 & 0 \\
0 & 0 & 0 & 0 & \frac{(1+2 v)}{2} & 0 \\
0 & 0 & 0 & 0 & 0 & \frac{(1+2 v)}{2}
\end{array}\right]
$$

$B_{L}$ is the matrix that maps nodal displacement to strain in the case of infinitesimal strain. Again, for the simple case of an elastic material $B_{L}$ is a $6 \times 3 n$ matrix, where $n$ is the number of nodes in the element.

$$
\mathbf{B}_{\mathbf{L}}=\mathbf{L N}
$$


Where $\boldsymbol{N}$ is the interpolation function. For a 20-node quadratic element, some of the shape functions have the following form in the natural coordinate system [45]:

$$
\begin{aligned}
& \mathbf{N}=\left[N_{1}, \ldots, N_{i}, \ldots, N_{20}\right]^{T} \\
& N_{i}=\left\{\begin{array}{c}
\frac{1}{8}[(1+\xi)(1-\eta)(1-\varsigma)(\xi-\eta-\varsigma-2)], i=1 \\
\ldots \\
\frac{1}{4}\left[\left(1-\varsigma^{2}\right)(1-\xi)(1-\eta)\right], i=20
\end{array}\right.
\end{aligned}
$$

Where $L$ in equation 2.6 is defined in equation 2.8. $L$ is a gradient function. For a system with three degree of freedom and six strain dimensions, $L$ has the form:

$$
\mathbf{L}^{T}=\left[\begin{array}{cccccc}
\partial / \partial x & 0 & 0 & \partial / \partial y & 0 & \partial / \partial z \\
0 & \partial / \partial y & 0 & \partial / \partial x & \partial / \partial z & 0 \\
0 & 0 & \partial / \partial z & 0 & \partial / \partial y & \partial / \partial x
\end{array}\right]
$$

Det $\boldsymbol{J}$ is the determinant of the Jacobian matrix that maps the deformed element geometry in the Cartesian coordinate system to the ordered matrix geometry in the natural coordinate system, as illustrated in Figure 2-8. This determinant is required to maintain the volume relation between the element in the deformed state to the element in the unreformed state, which is done through a scaling factor known as the determinant of the Jacobian. 

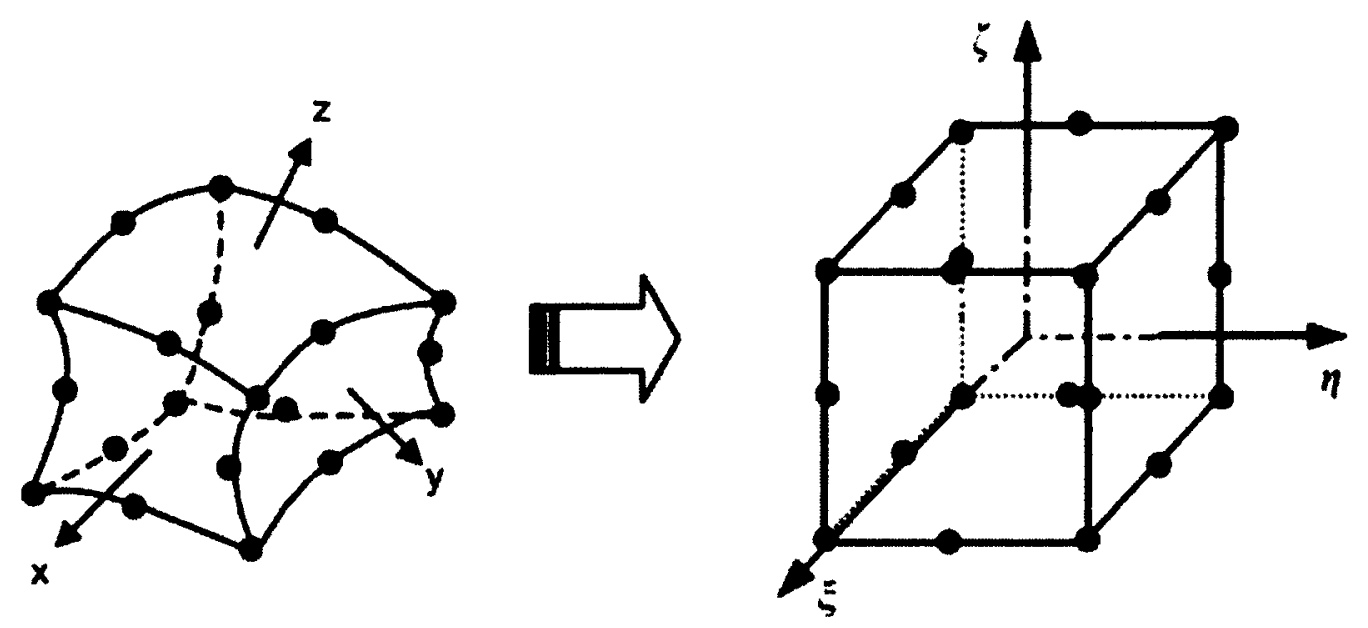

Figure 2-8: Jacobian transformation [46]

A three-dimensional Jacobian matrix has the form [46]:

$$
\mathbf{J}=\left[\begin{array}{lll}
\partial x / \partial \xi & \partial x / \partial \eta & \partial x / \partial \zeta \\
\partial y / \partial \xi & \partial y / \partial \eta & \partial y / \partial \zeta \\
\partial z / \partial \xi & \partial z / \partial \eta & \partial z / \partial \zeta
\end{array}\right]=\sum_{i}\left[\begin{array}{lll}
\frac{\partial N_{i}}{\partial \xi} x_{i} & \frac{\partial N_{i}}{\partial \eta} x_{i} & \frac{\partial N_{i}}{\partial \zeta} x_{i} \\
\frac{\partial N_{i}}{\partial \xi} y_{i} & \frac{\partial N_{i}}{\partial \eta} y_{i} & \frac{\partial N_{i}}{\partial \zeta} y_{i} \\
\frac{\partial N_{i}}{\partial \xi} z_{i} & \frac{\partial N_{i}}{\partial \eta} z_{i} & \frac{\partial N_{i}}{\partial \zeta} z_{i}
\end{array}\right]
$$

In order to undertake the integration of Equation 2.3 , the matrix must be evaluated using a Gaussian integration scheme. The integration is replaced by a summation. The values of the terms of the summation are evaluated at a series of Gauss points and are multiplied by weight factor, $\mathrm{w}_{(\mathrm{r}, \mathrm{s}, \mathrm{t})}$ :

$$
\begin{aligned}
\mathbf{K}_{\mathbf{L}} & =\int_{V} \mathbf{B}_{\mathbf{L}}{ }^{T} \mathbf{D B}_{\mathbf{L}} d V \\
& =\int_{-1}^{1} \int_{-1}^{1} \int_{-1}^{1} \mathbf{B}_{\mathbf{L}}{ }^{T} \mathbf{D B}_{\mathbf{L}} \operatorname{det} \mathbf{J} d \xi d \eta d \zeta \\
& =\sum_{r} \sum_{s} \sum_{t}\left(w_{(r, s, t)} \mathbf{B}_{\mathbf{L}}{ }^{T} \mathbf{D} \mathbf{B}_{\mathbf{L}}|\operatorname{det} \mathbf{J}|_{(r, s, t)}\right)
\end{aligned}
$$


The Gaussian integration scheme for a 20-node quadratic brick element C3D20 is illustrated in Figure 2-9. The location and weight factor of some of the Gaussian integration points are listed in Table 2-1.
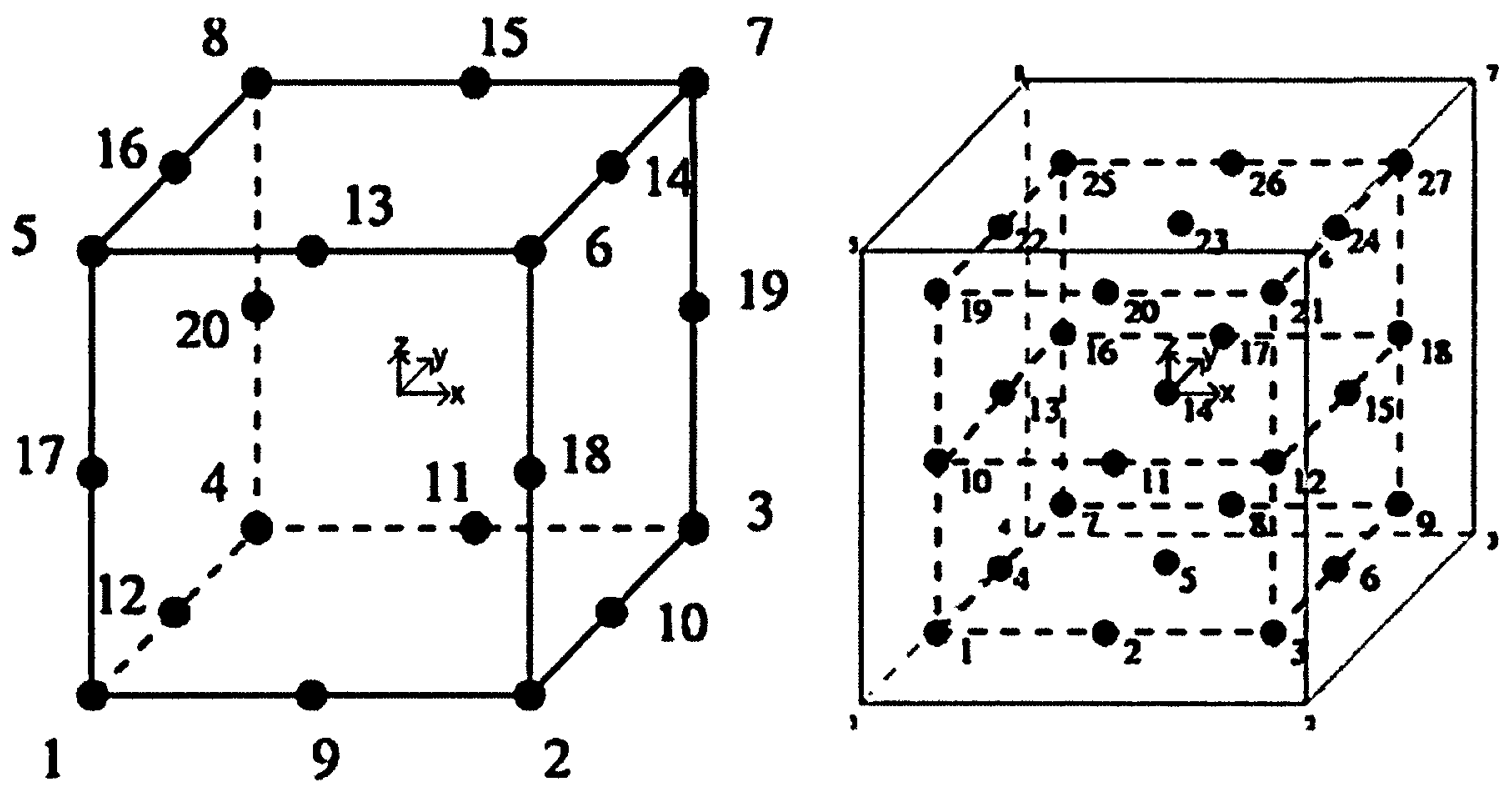

Figure 2-9: Gaussian integration scheme [47]

Table 2-1: Examples of Gauss points and weight factors for 20-node quadratic brick element C3D20 [44]

\begin{tabular}{c|c|c|c|c}
\hline Gauss Point & \multicolumn{3}{|c|}{ Location } & Weight Factor \\
& $\mathrm{X}$ & $\mathrm{Y}$ & $\mathrm{Z}$ & \\
\hline 1 & -0.77459667 & -0.77459667 & -0.77459667 & 0.171467764 \\
2 & 0.77459667 & 0 & -0.77459667 & 0.274348422 \\
5 & 0 & 0 & -0.77459667 & 0.438957476 \\
14 & 0 & 0 & 0 & 0.702331962
\end{tabular}




\subsubsection{Large Displacement Stiffness Matrix, $K_{\mathrm{NL}}$}

An object undergoing large deformations, displacements and rotations is illustrated in Figure 2-10. This object's initial configuration, $x^{0}$, is transformed to the current configuration, $x$, through a system of displacements, $u$ and $u+d u$. Point $P$ in the current configuration is identified through vector $x$. Neighboring points, $q$ are identified by the differential vector $d x[43]$.

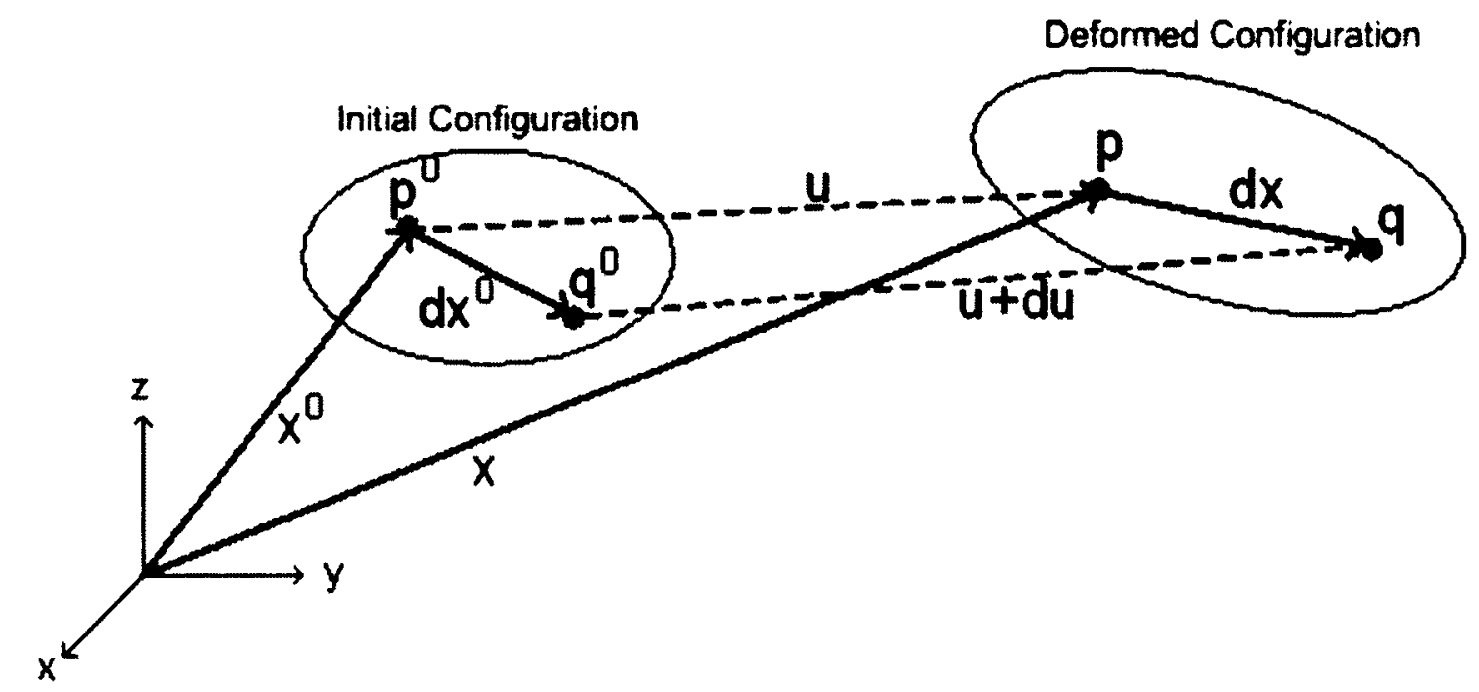

Figure 2-10: Initial configuration, $x^{0}$ and deformed configuration, $x$ [43]

The relationship between the initial configuration differential vector, $d x^{0}$, and the current configuration differential vector, $d x$, is mapped in the deformation gradient, $F$ [43]:

$$
\mathbf{F}=\frac{\partial x}{\partial x^{0}}=\left[\begin{array}{lll}
\partial x / \partial x^{0} & \partial x / \partial y^{0} & \partial x / \partial z^{0} \\
\partial y / \partial x^{0} & \partial y / \partial y^{0} & \partial y / \partial z^{0} \\
\partial z / \partial x^{0} & \partial z / \partial y^{0} & \partial z / \partial z^{0}
\end{array}\right]=I+\nabla u
$$

where $\nabla u$ is the displacement gradient: 


$$
\nabla \mathbf{u}=\left[\begin{array}{lll}
\partial u / \partial x & \partial u / \partial y & \partial u / \partial z \\
\partial v / \partial x & \partial v / \partial y & \partial v / \partial z \\
\partial w / \partial x & \partial w / \partial y & \partial w / \partial z
\end{array}\right]
$$

$F$ reflects the deformation, displacement and rotations of the object and is a function of $I$ and $\nabla u$. However, strains are only a function of $\nabla u$. Strains are not a function of displacement or rotations alone.

The deformation gradient can be modified with the gradient function, to give $\overline{\mathbf{F}}$ [43]. $\overline{\mathbf{F}}$ is used to compute $\overline{\mathbf{K}}$.

$$
\begin{aligned}
& \overline{\mathbf{F}}=\mathbf{F L x}=\left[\begin{array}{lll}
\partial x / \partial x^{0} & \partial x / \partial y^{0} & \partial x / \partial z^{0} \\
\partial y / \partial x^{0} & \partial y / \partial y^{0} & \partial y / \partial z^{0} \\
\partial z / \partial x^{0} & \partial z / \partial y^{0} & \partial z / \partial z^{0}
\end{array}\left[\begin{array}{ccc}
\partial / \partial x & 0 & 0 \\
0 & \partial / \partial y & 0 \\
0 & 0 & \partial / \partial z \\
\partial / \partial y & \partial / \partial x & 0 \\
0 & \partial / \partial z & \partial / \partial y \\
\partial / \partial z & 0 & \partial / \partial x
\end{array}\right] x\left[\begin{array}{ccc}
\partial / \partial x & 0 & 0 \\
0 & \partial / \partial y & 0 \\
0 & 0 & \partial / \partial z \\
\partial / \partial y & \partial / \partial x & 0 \\
0 & \partial / \partial z & \partial / \partial y \\
\partial / \partial z & 0 & \partial / \partial x
\end{array}\right],\left[\begin{array}{ccc}
\partial / \partial x & 0 & 0 \\
0 & \partial / \partial y & 0 \\
0 & 0 & \partial / \partial z \\
\partial / \partial y & \partial / \partial x & 0 \\
0 & \partial / \partial z & \partial / \partial y \\
\partial / \partial z & 0 & \partial / \partial x
\end{array}\right] z\right] \\
& \overline{\mathbf{F}}=\left[\begin{array}{ccccccccc}
\partial x / \partial x^{0} & 0 & 0 & \partial y / \partial x^{0} & 0 & 0 & \partial z / \partial x^{0} & 0 & 0 \\
0 & \partial x / \partial y^{0} & 0 & 0 & \partial y / \partial y^{0} & 0 & 0 & \partial z / \partial y^{0} & 0 \\
0 & 0 & \partial x / \partial z^{0} & 0 & 0 & \partial y / \partial z^{0} & 0 & 0 & \partial z / \partial z^{0} \\
\partial x / \partial y^{0} & \partial x / \partial x^{0} & 0 & \partial y / \partial y^{0} & \partial y / \partial x^{0} & 0 & \partial z / \partial y^{0} & \partial z / \partial x^{0} & 0 \\
0 & \partial x / \partial z^{0} & \partial x / \partial y^{0} & 0 & \partial y / \partial z^{0} & \partial y / \partial y^{0} & 0 & \partial z / \partial z \nu & \partial z / \partial y^{0} \\
\partial x / \partial z^{0} & 0 & \partial x / \partial x^{0} & \partial y / \partial z^{0} & 0 & \partial y / \partial x^{0} & \partial z / \partial z^{0} & 0 & \partial z / \partial x^{0}
\end{array}\right]
\end{aligned}
$$

The large displacement stiffness matrix, $\overline{\mathbf{K}}$, relates load, $\boldsymbol{P}$, and displacement, $u$, for the case where the element is undergoing large displacements or rotations. The matrix has the form:

$$
\overline{\mathbf{K}}=\mathbf{K}_{\mathbf{L}}+\mathbf{K}_{\mathrm{NL}}=\int_{V} \mathbf{B} \overline{\mathbf{F}}^{\mathrm{T}} \mathbf{D} \overline{\mathbf{F}} \mathbf{B} d V
$$

Where $B$ is the matrix that maps nodal degrees of freedom to the displacement gradient. For a three degree of freedom system with $n$ nodes, $B$ has the form: 


$$
\left.\mathbf{B}=\mathbf{L}_{\mathbf{N L}} \mathbf{N}=\left[\begin{array}{ccc}
\partial / \partial x^{0} & 0 & 0 \\
\partial / \partial y^{0} & 0 & 0 \\
\partial / \partial z^{0} & 0 & 0 \\
0 & \partial / \partial x^{0} & 0 \\
0 & \partial / \partial y^{0} & 0 \\
0 & \partial / \partial z^{0} & 0 \\
0 & 0 & \partial / \partial x^{0} \\
0 & 0 & \partial / \partial y^{0} \\
0 & 0 & \partial / \partial z^{0}
\end{array}\right] N_{1} \ldots\left[\begin{array}{ccc}
\partial / \partial x^{0} & 0 & 0 \\
\partial / \partial y^{0} & 0 & 0 \\
\partial / \partial z^{0} & 0 & 0 \\
0 & \partial / \partial x^{0} & 0 \\
0 & \partial / \partial y^{0} & 0 \\
0 & \partial / \partial z^{0} & 0 \\
0 & 0 & \partial / \partial x^{0} \\
0 & 0 & \partial / \partial y^{0} \\
0 & 0 & \partial / \partial z^{0}
\end{array}\right] N_{n}\right]
$$

\subsubsection{Geometric Stiffness Matrix, $K_{\sigma}$}

The geometric stiffness matrix relates load on the element and displacement for the case where the stiffness of the element is a function of the load acting upon it, such as in the case where the element is experiencing large stresses. The matrix has the form [48]:

$$
\mathbf{K}_{\mathbf{a}}=\int_{V} \mathbf{B}^{T}\left[\begin{array}{ccc}
\hat{\mathbf{s}} & 0 & 0 \\
0 & \hat{\mathbf{s}} & 0 \\
0 & 0 & \hat{\mathbf{s}}
\end{array}\right] \mathbf{B} d V
$$

Where $\hat{s}$ is the second Piola-Kirchoff stress tensor [43]:

$$
\hat{\mathbf{s}}=\operatorname{det} \mathbf{F} \mathbf{F}^{-1} \mathbf{T F}^{\mathbf{T}}=\operatorname{det} \mathbf{F} \mathbf{F}^{-1}\left[\begin{array}{ccc}
T_{x} & \tau_{x y} & \tau_{z x} \\
\tau_{x y} & T_{y} & \tau_{y z} \\
\tau_{z x} & \tau_{y z} & T_{z}
\end{array}\right] \mathbf{F}^{T}
$$

The stresses in the element are determined from the strains. The strains in the element are determined from the deformation gradient, $F$ : 


$$
\begin{aligned}
& \mathbf{T}=\mathbf{E} \varepsilon \\
& \boldsymbol{\varepsilon}=\left[\begin{array}{lll}
\varepsilon_{x x} & \varepsilon_{x y} & \varepsilon_{x z} \\
\varepsilon_{y x} & \varepsilon_{y y} & \varepsilon_{y z} \\
\varepsilon_{z x} & \varepsilon_{z y} & \varepsilon_{z z}
\end{array}\right]=\frac{1}{2}\left(\nabla \mathbf{u}^{T}+\nabla \mathbf{u}+\nabla \mathbf{u}^{T} \nabla \mathbf{u}\right)=\frac{1}{2}\left[\mathbf{F}^{\mathrm{T}} \mathbf{F}-\mathbf{I}\right]
\end{aligned}
$$

In order to compute the stress or strain relationship, the simulation must have completed at least one iteration in the solution procedure.

\subsection{Solution Methods}

There are a number of techniques used to solve nonlinear finite element analyses, including: the incremental (Euler) method, the iterative (Newton-Raphson) method, the corrected incremental method or the modified Newton-Raphson method.

The response curve of the system being analyzed (shown in Figure 2-11) maps the relationship between load, $P$ and displacement, $u$. Many of the solution schemes rely on finding the tangent to this curve known as the tangent stiffness matrix, $k_{t}$. The tangential stiffness matrix represents the local tangent to the response curve [49]:

$$
\left(k_{t}\right)_{i}=\left(\frac{\partial P}{\partial u}\right)_{i}
$$

\subsubsection{Incremental (Euler) Method}

The incremental (Euler) method is illustrated in Figure 2-11. Given a target load, $\Delta P_{B}$, the system is subjected to a series of incremental loads $\Delta P_{i}$. For each incremental load applied, the system subsequently undergoes an incremental displacement response, $\Delta u_{i}$. 
The magnitude of the incremental displacement is proportional to the incremental load and the inverse of the tangent stiffness matrix, $\left(k_{t}\right)_{i}$ [49].

$$
u_{i+1}=u_{i}+\Delta u_{i}=u_{i}+\left[\left(\frac{\Delta u}{\Delta p}\right)_{i} \Delta P_{i+1}\right]=u_{i}+\left(\left(k_{t}\right)_{i}^{-1} \Delta P_{i+1}\right)
$$

Once the sum of the incremental loads equals the target load for the system, the sum of the incremental displacements undergone by the system should be equal to the displacement of the system when subjected to the target load [49]. Unfortunately, the incremental method solution response will always drift from the system response [49], as shown in Figure 2-11. This drift can be reduced with the use of a corrective term. The corrected incremental method is discussed below.

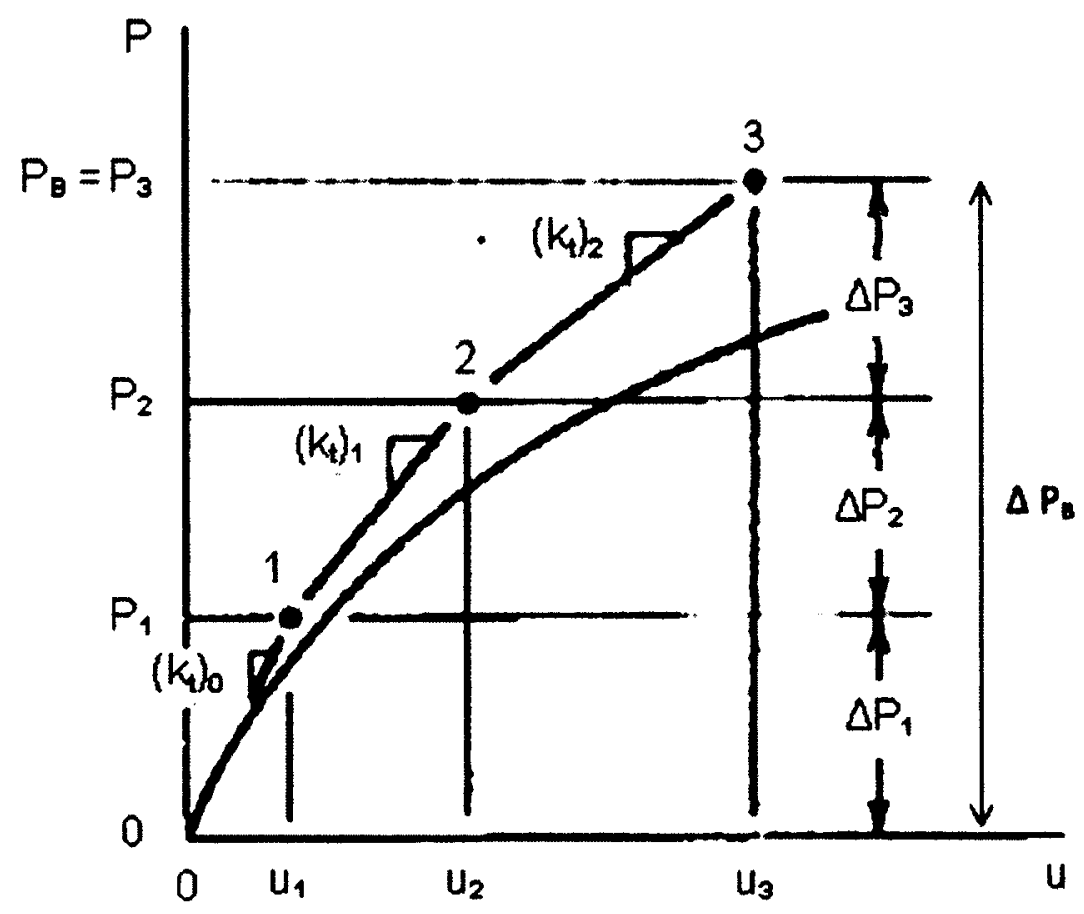

Figure 2-11: Incremental (Euler) method [48] 


\subsubsection{Iterative Method (Newton-Raphson) Method}

The Newton-Raphson Method is illustrated in Figure 2-12. At a given starting point, $A$ (Figure 2-12 (A)) and a starting load, $P_{A}$, the resulting response displacement of the system due to $P_{A}$ is measured to be $u_{A}$ and the tangential stiffness matrix at point $A,\left(k_{v}\right)_{A}$ is determined. The target load is $P_{B}$. The displacement $u_{B}$ that would result from load $P_{B}$ is sought. A truncated Taylor series of about $A$ is then [48]:

$$
P_{B}=f\left(u_{B}\right)=f\left(u_{A}+\Delta u\right)=P_{A}+\left(\frac{\partial P}{\partial u}\right)_{A} \Delta u=P_{A}+\left(k_{t}\right)_{A}^{-1} \Delta u
$$

The residual or out-of-balance load term, $g$, is a measure of the difference between the target load, $P_{B}$, and the internal load that would produce the iterated displacement, $P_{i}=f\left(u_{i}\right)[48]:$

$$
r\left(u_{i}\right)=P_{B}-P_{i}\left(u_{i}\right)
$$

The next displacement iteration, $u_{i+1}$, occurs at the intercept of $P_{\mathrm{B}}$ and the tangent from current load iteration $\mathrm{P}_{i}\left(\mathrm{u}_{i}\right)[48]$ :

$$
u_{i+1}=u_{i}+K_{i}^{-1} r=u_{i}+K_{i}^{-1}\left(P_{B}-P_{i}\left(u_{i}\right)\right)
$$

The iteration is repeated until the residual, $r$, is zero or is sufficiently small. 


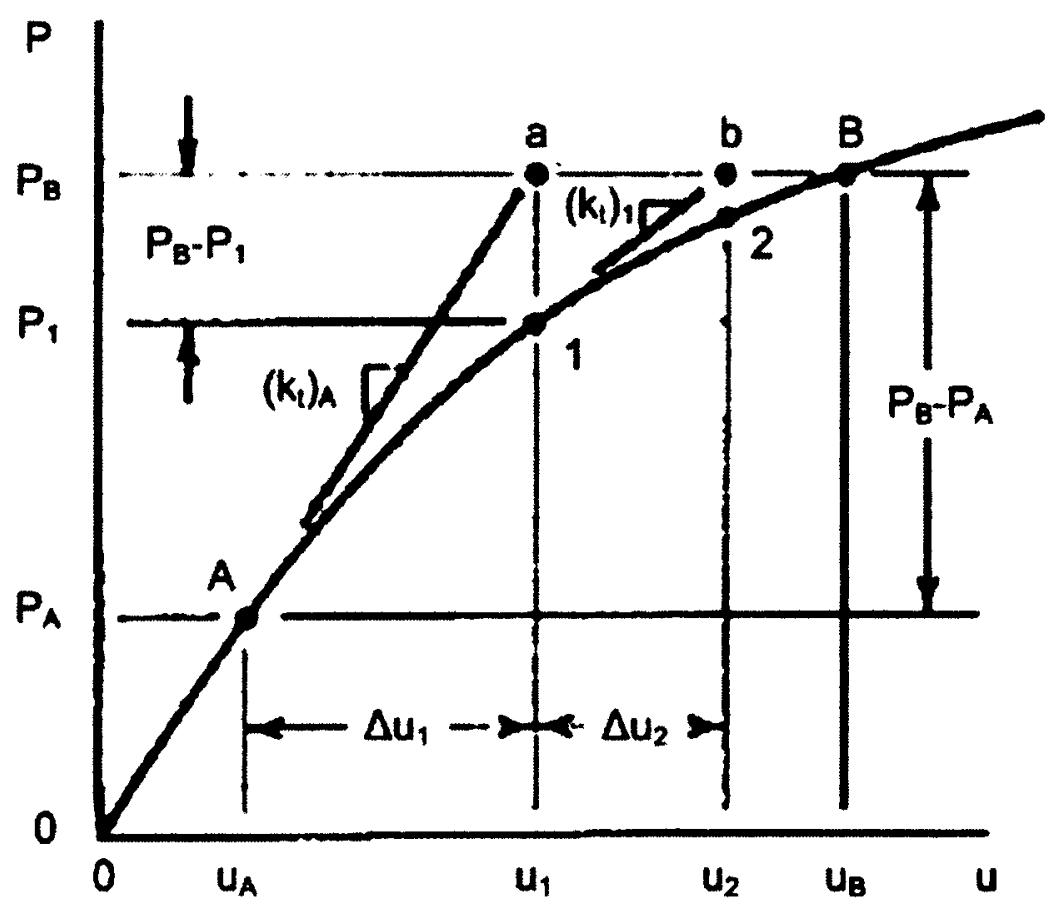

Figure 2-12: Newton-Raphson method [48]

The Newton-Raphson Method seeks to eliminate the out-of-balance force term, $g$. The seeking process may take many iterations. The number of iterations required may be reduced by accepting an out-of-balance load that is "sufficiently small". Accepting such imperfections may introduce a significant cumulative error into complex systems that rely on many iterative processes.

\subsubsection{Corrected Incremental Method}

The response solution given by the Incremental Method will lead to drift from the system response. This drift can be reduced or eliminated with the use of a corrective term. The 
corrected incremental displacement incorporates the residual force term from NewtonRaphson method [48]:

$$
r_{i}\left(u_{i}\right)=P_{\text {External }}-P_{\text {Internal }}\left(u_{i}\right)
$$

$g_{i}$ is the same term introduced in the Newton-Raphson method. $r_{i}$ is the residual load term (i.e. difference between the load $P_{E}$ and the load that would produce displacement $\left.u_{i}, P_{i}\left(u_{i}\right).\right)$

For a given state load, $P_{i}$, and displacement, $u_{i}$, the stiffness matrix, $\left(k_{\jmath}\right)_{i}$ and residual load, $r_{i}$ are determined. The increase in displacement due to an applied load is proportional to the stiffness matrix and the difference between the residual [48]:

$$
u_{i+1}=u_{i}+\left(k_{t}\right)_{i}^{-1}\left(P_{i+1}-P_{i R}\right)=u_{i}+\left(k_{t}\right)_{i}^{-1}\left[P_{i+1}-\left(P_{i}-r_{i}\right)\right]
$$

The response scheme given by the corrected incremental method is illustrated in Figure 2-14. 


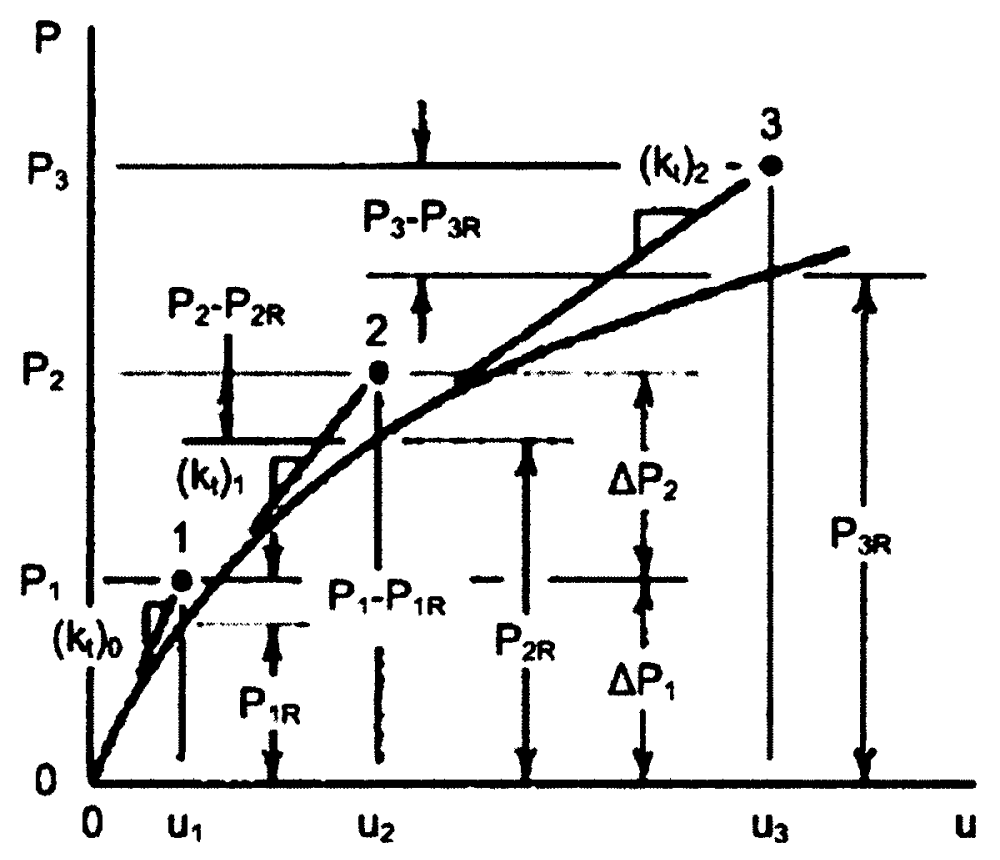

Figure 2-13: Incremental method with load correction [48]

The corrected incremental method requires less incrimination and delivers a response that more closely follows the system response than the original, uncorrected incremental method.

The difference between the Newton-Raphson Method and the Corrected Incremental Method is thus: The Newton Raphson Method iterated displacement until the load that would produce such displacement reached the target load. The Corrected Incremental Method increments load until the target load is reached and the resulting displacement is measured. 


\section{Chapter 3: Validation}

\subsection{Introduction}

A validation of the capabilities of the ABAQUS finite element analysis software and of the capabilities of the finite element analyst was undertaken. This validation took the form of a series of linear elastic tests, geometric nonlinear elastic tests and piezoelectric tests. Linear elastic tests were furnished by MacNeal and Harder [50]. Geometric nonlinear elastic test were furnished by Sze [51] and by Benham and Crawford [42]. Piezoelectric tests were furnished by Martinez [44] and by the reproduction of experiments conducted in [4].

ABAQUS, the finite element analysis software package, was used for all tests. Testing was undertaken using the ABAQUS 20-node elastic element (C3D20), 20-node elastic reduced-order-integration element (C3D20R) and the 20-node piezoelectric element (C3D20E).

\subsection{Linear Elastic Problems: MacNeal and Harder Tests}

MacNeal and Harder established a set of tests to determine the accuracy of finite element analysis tools in various applications [50]. The tests consisted of simple structures with geometries undergoing simple load cases that are easily solved analytically for which

reliable computational results are available. These simple problems test the capability of 
the software and of the analyst to implement simple geometries, simple boundary conditions and simple load cases. Furthermore, the tests evaluate the capability of the software to correctly implement element discretization, assembly, processing and postprocessing.

MacNeal and Harder provided theoretical results for each test presented in [50]. Agreement with these theoretical results is the metric used to gauge the success of the ABAQUS analysis. In some cases, these theoretical results are unachievable due to sparse meshing. MacNeal and Harder also provided the most accurate results achievable with sparse meshing. Achieving results that are comparable or better than those obtained by MacNeal and Harder $(\mathrm{M}+\mathrm{H})$ is the secondary metric used to gauge the success of the ABAQUS analysis.

The MacNeal and Harder problem sets have been nondimensionalized. While not expressed, a self-consistent system of units are used. The magnitude and order of the values presented are consistent.

The MacNeal and Harder tests were undertaken using the ABAQUS 20-node elastic element (C3D20), the 20-node reduced-order-integration element (C3D20R) and the 20node piezoelectric element (C3D20E). The C3D20E element returned results that were identical to those returned by the C3D20 element and are not presented.

The tests undertaken include:

- Patch test

- Straight beam test (with rectangular, parallelogram and trapezoidal elements)

- Curved beam test 
- Twisted beam test

- Rectangular plate test

- Scordelis-Lo roof test

\subsubsection{Patch Test}

The Patch Test is designed to be simple, yet test all features of Finite Element Analysis: application of boundary conditions, meshing, element interaction, solution and postprocessing.

The patch test consisted of a unit cube. The cube was assigned a Young's modulus $1.0 \times 10^{6}$ and a Poisson's ratio of 0.25 . The mesh was assigned a geometry defined by the cube geometry and a series of internal nodes. The elements of the patch test as well as the coordinates of the internal nodes are included in Figure 3-1.

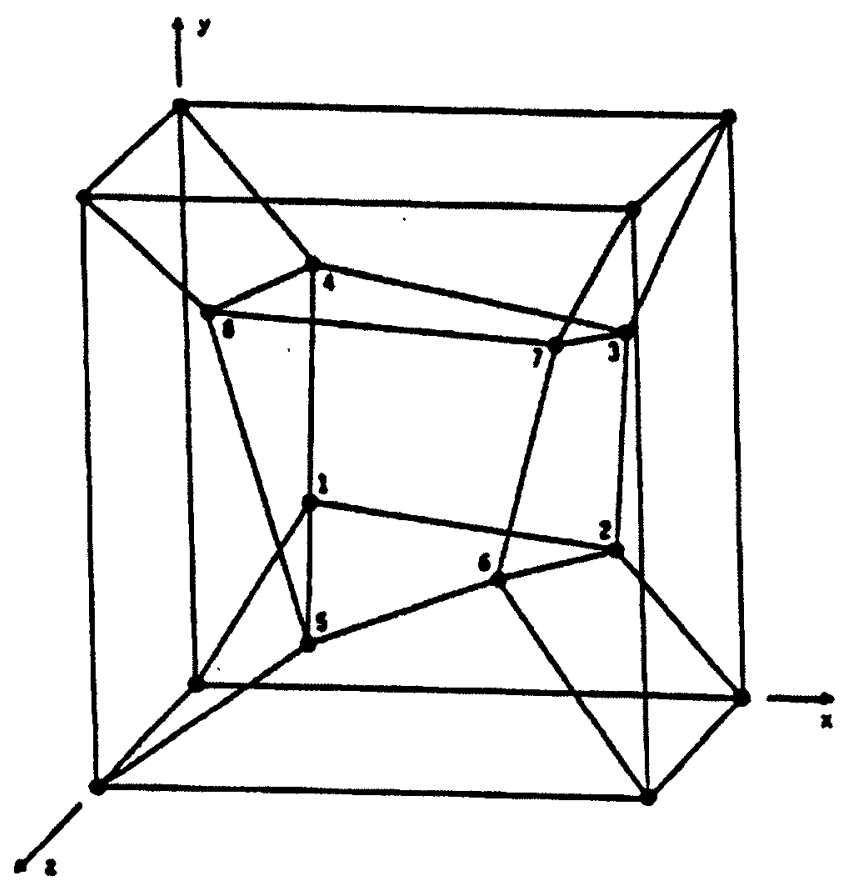

Coordinates:

\begin{tabular}{|c|c|c|c|}
\hline & $\mathrm{x}$ & $\mathrm{y}$ & $\mathrm{z}$ \\
\hline 1 & 0.249 & 0.342 & 0.192 \\
\hline 2 & 0.826 & 0.288 & 0.288 \\
\hline 3 & 0.850 & 0.649 & 0.263 \\
\hline 4 & 0.273 & 0.750 & 0.230 \\
\hline 5 & 0.320 & 0.186 & 0.643 \\
\hline 6 & 0.677 & 0.305 & 0.683 \\
\hline 7 & 0.788 & 0.693 & 0.644 \\
\hline 8 & 0.165 & 0.745 & 0.702 \\
\hline
\end{tabular}

Figure 3-1: Patch test geometry and nodal coordinates [50] 
A displacement field was assigned to the exterior nodes of the structure as follows:

$$
\begin{aligned}
& u=\frac{(2 x+y+z)}{2} \cdot 10^{-3} \\
& v=\frac{(x+2 y+z)}{2} \cdot 10^{-3} \\
& w=\frac{(x+y+2 z)}{2} \cdot 10^{-3}
\end{aligned}
$$

The theoretical resultant stress and strain fields are as follows:

$$
\begin{aligned}
& \varepsilon_{x}=\varepsilon_{y}=\varepsilon_{z}=\gamma_{x y}=\gamma_{y z}=\gamma_{z x}=10^{-3} \\
& \sigma_{x}=\sigma_{y}=\sigma_{z}=2000 \\
& \tau_{x y}=\tau_{y z}=\tau_{z x}=400
\end{aligned}
$$

The results of the patch test are tabulated in Table 3-1.

Table 3-1: Results for patch test

\begin{tabular}{|l|l|l|l|l|}
\hline \multicolumn{5}{|c|}{ Element C3D20 } \\
\hline Load & ABAQUS Result & Theoretical Result & $\%$ Diff & Agree? \\
\hline Stress & 2000 & 2000 & 0.00 & Yes \\
\hline Shear & 400 & 400 & 0.00 & Yes \\
\hline Strains & $1.00 \mathrm{E}-03$ & $1.00 \mathrm{E}-03$ & 0.00 & Yes \\
\hline
\end{tabular}

\begin{tabular}{|l|l|l|l|l|}
\hline \multicolumn{5}{|c|}{ Element C3D20R } \\
\hline & ABAQUS Result & Theoretical Result & $\%$ Diff & Agree? \\
\hline Stress & 2000 & 2000 & 0.00 & Yes \\
\hline Shear & 400 & 400 & 0.00 & Yes \\
\hline Strains & $1.00 \mathrm{E}-03$ & $1.00 \mathrm{E}-03$ & 0.00 & Yes \\
\hline
\end{tabular}

The finite element analysis of the patch test provided results that were consistent with the predicted results. ABAQUS passed the patch test. 


\subsubsection{Straight Beam Test}

The straight beam test consisted of a straight beam constructed from rectangular, parallelogram and trapezoidal elements.

The beam had dimensions of $6.0 \times 0.2 \times 0.1$. The beam was assigned a mesh that was subdivided into six elements along its length. The beam geometry is depicted in Figure 32. The beam was assigned a Young's modulus of $1.0 \times 10^{7}$ and a Poisson's ratio of 0.3. The beam was fully constrained at one end and was subjected to a unit load in either the axial, lateral out-of-plane or lateral in-plane directions. The tip displacement due to this load was measured. The tip deflections are tabulated Table 3-2.

a)

b)

c)

Figure 3-2: Straight beam, with

a) Rectangular elements

b) Parallelogram elements

c) Trapezoidal elements

Table 3-2: Results for the straight beam test

\begin{tabular}{|c|c|c|c|c|c|c|c|}
\hline \multicolumn{8}{|c|}{ Rectangular Elements } \\
\hline \multicolumn{8}{|c|}{ Element C3D20 } \\
\hline & ABAQUS & \multicolumn{3}{|c|}{ Theoretical } & \multicolumn{3}{|c|}{$\mathrm{M}+\mathrm{H}$} \\
\hline Load & $\begin{array}{l}\text { Tip } \\
\text { Displacement }\end{array}$ & $\begin{array}{l}\text { Tip } \\
\text { Displacement }\end{array}$ & \begin{tabular}{|l|}
$\%$ \\
Diff
\end{tabular} & Agree? & $\begin{array}{l}\text { Tip } \\
\text { Displacement }\end{array}$ & $\begin{array}{l}\text { \% } \\
\text { Diff }\end{array}$ & Agree? \\
\hline Extension & $2.983 \mathrm{E}-05$ & $3.00 \mathrm{E}-05$ & 0.57 & Yes & $2.98 \mathrm{E}-05$ & 0.10 & Yes \\
\hline In-Plane & $1.049 \mathrm{E}-01$ & 0.1081 & 2.96 & Yes & $1.05 \mathrm{E}-01$ & 0.10 & Yes \\
\hline $\begin{array}{l}\text { Out-of- } \\
\text { Plane }\end{array}$ & $4.151 \mathrm{E}-01$ & 0.4321 & 3.93 & Yes & 4.15E-01 & 0.02 & Yes \\
\hline
\end{tabular}




\begin{tabular}{|c|c|c|c|c|c|c|c|}
\hline \multicolumn{8}{|c|}{ Element C3D20R } \\
\hline & ABAQUS & \multicolumn{3}{|c|}{ Theoretical } & \multicolumn{3}{|c|}{$\mathrm{M}+\mathrm{H}$} \\
\hline Load & $\begin{array}{l}\text { Tip } \\
\text { Displacement }\end{array}$ & $\begin{array}{l}\text { Tip } \\
\text { Displacement }\end{array}$ & \begin{tabular}{|l|}
$\%$ \\
Diff \\
\end{tabular} & Agree? & $\begin{array}{l}\text { Tip } \\
\text { Displacement }\end{array}$ & $\begin{array}{l}\% \\
\text { Diff } \\
\end{array}$ & Agree? \\
\hline Extension & $2.997 \mathrm{E}-05$ & $3.00 \mathrm{E}-05$ & 0.10 & Yes & $3.00 \mathrm{E}-05$ & 0.10 & Yes \\
\hline In-Plane & $1.049 \mathrm{E}-01$ & 0.1081 & 2.96 & Yes & $1.05 \mathrm{E}-01$ & 0.10 & Yes \\
\hline $\begin{array}{l}\text { Out-of- } \\
\text { Plane }\end{array}$ & 4.151E-01 & 0.4321 & 3.93 & Yes & $4.15 \mathrm{E}-01$ & 0.02 & Yes \\
\hline
\end{tabular}

\begin{tabular}{|l|l|l|l|l|l|l|l|}
\hline \multicolumn{9}{|c|}{ Parallelogram Elements } \\
\hline & \multicolumn{1}{|c|}{ ABAQUS } & \multicolumn{1}{|c|}{ Theoretical } & \multicolumn{3}{c|}{ M+H } \\
\hline & $\begin{array}{l}\text { Tip } \\
\text { Displacement }\end{array}$ & $\begin{array}{l}\text { Tip } \\
\text { Displacement }\end{array}$ & $\begin{array}{l}\% \\
\text { Diff }\end{array}$ & Agree? & $\begin{array}{l}\text { Tip } \\
\text { Displacement }\end{array}$ & $\begin{array}{l}\% \\
\text { Diff }\end{array}$ & Agree? \\
\hline Extension & 2.87 E-05 & $3.00 \mathrm{E}-05$ & 4.33 & Yes & $2.83 \mathrm{E}-05$ & 1.41 & Yes \\
\hline In-Plane & $1.05 \mathrm{E}-01$ & 0.1081 & 2.87 & Yes & $1.04 \mathrm{E}-01$ & 0.96 & Yes \\
\hline $\begin{array}{l}\text { Out-of- } \\
\text { Plane }\end{array}$ & $4.13 \mathrm{E}-01$ & 0.4321 & 4.42 & Yes & $3.76 \mathrm{E}-01$ & 9.84 & No \\
\hline
\end{tabular}

\begin{tabular}{|c|c|c|c|c|c|c|c|}
\hline \multicolumn{8}{|c|}{ Element C3D20R } \\
\hline & ABAQUS & Theoretical & & & & $+\mathrm{H}$ & \\
\hline Load & $\begin{array}{l}\text { Tip } \\
\text { Displacement }\end{array}$ & $\begin{array}{l}\text { Tip } \\
\text { Displacement }\end{array}$ & $\begin{array}{l}\% \\
\text { Diff }\end{array}$ & Agree? & $\begin{array}{l}\text { Tip } \\
\text { Displacement }\end{array}$ & $\begin{array}{l}\% \\
\text { Diff }\end{array}$ & Agree? \\
\hline Extension & $3.00 \mathrm{E}-05$ & \begin{tabular}{|l|}
$3.00 \mathrm{E}-05$ \\
\end{tabular} & 0.00 & Yes & $3.00 \mathrm{E}-05$ & 0.00 & Yes \\
\hline In-Plane & $1.07 \mathrm{E}-01$ & 0.1081 & 1.02 & Yes & \begin{tabular}{|l|}
$1.05 \mathrm{E}-01$ \\
\end{tabular} & 1.90 & Yes \\
\hline $\begin{array}{l}\text { Out-of- } \\
\text { Plane }\end{array}$ & 4.15E-01 & 0.4321 & 3.96 & Yes & 4.13E-01 & 0.48 & Yes \\
\hline
\end{tabular}

\begin{tabular}{|l|l|l|l|l|l|l|l|}
\hline \multicolumn{9}{|c|}{ Trapezoidal Elements } \\
\hline & \multicolumn{9}{|c|}{ Theoretical } & \multicolumn{3}{c|}{ M+H } \\
\hline & ABAQUS & \multicolumn{3}{c|}{ Tip 20} \\
\hline Load & Displacement & $\begin{array}{l}\text { Tip } \\
\text { Displacement }\end{array}$ & $\begin{array}{l}\% \\
\text { Diff }\end{array}$ & Agree? & $\begin{array}{l}\text { Tip } \\
\text { Displacement }\end{array}$ & $\begin{array}{l}\% \\
\text { Diff }\end{array}$ & Agree? \\
\hline Extension & $2.983 \mathrm{E}-05$ & $3.00 \mathrm{E}-05$ & 0.57 & Yes & $2.98 \mathrm{E}-05$ & 0.10 & Yes \\
\hline In-Plane & $1.043 \mathrm{E}-01$ & 0.1081 & 3.52 & Yes & $1.04 \mathrm{E}-01$ & 0.29 & Yes \\
\hline $\begin{array}{l}\text { Out-of- } \\
\text { Plane }\end{array}$ & $3.765 \mathrm{E}-01$ & 0.4321 & 12.87 & No & $3.77 \mathrm{E}-01$ & 0.13 & Yes \\
\hline
\end{tabular}




\begin{tabular}{|l|l|l|l|l|l|l|l|}
\hline & \multicolumn{9}{|c|}{ Element C3D20R } & \multicolumn{3}{c|}{ M+H } \\
\hline & ABAQUS & \multicolumn{3}{c|}{ Theoretical } & Tip & $\%$ & A \\
Load & $\begin{array}{l}\text { Tip } \\
\text { Displacement }\end{array}$ & $\begin{array}{l}\text { Tip } \\
\text { Displacement }\end{array}$ & $\begin{array}{l}\% \\
\text { Diff }\end{array}$ & Agree? & Displacement & Diff & Agree? \\
\hline Extension & $2.998 \mathrm{E}-05$ & $3.00 \mathrm{E}-05$ & 0.07 & Yes & $3.00 \mathrm{E}-05$ & 0.07 & Yes \\
\hline In-Plane & $1.043 \mathrm{E}-01$ & 0.1081 & 3.52 & Yes & $1.04 \mathrm{E}-01$ & 0.29 & Yes \\
\hline $\begin{array}{l}\text { Out-of- } \\
\text { Plane }\end{array}$ & $3.765 \mathrm{E}-01$ & 0.4321 & 12.87 & No & $3.77 \mathrm{E}-01$ & 0.13 & Yes \\
\hline
\end{tabular}

The models returned results that were consistent with the results set out in [50] or with those set out by $M+H$. ABAQUS passed the straight beam test.

\subsubsection{Curved Beam Test}

The curved beam test consisted of applying load to the free end of a curved beam. The beam had a cross-section of $0.2 \times 0.1$ and an arc-length of $90^{\circ}$, as illustrated in Figure 33. The beam was assigned a Young's modulus of $1.0 \times 10^{7}$ and a Poisson's ratio of 0.25 . The bean was assigned a mesh that was sectioned into six elements along its length. The beam was subjected to a unit load at the free end. The tip displacements resulting from this load are tabulated in Table 3-3. 


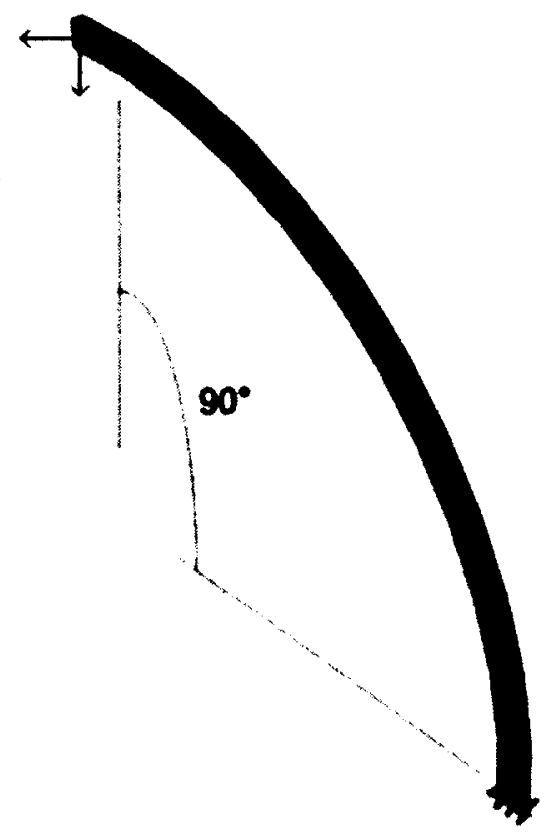

Figure 3-3: Curved beam [50]

Table 3-3: Results for the curved beam test

\begin{tabular}{|l|l|l|l|l|l|l|l|}
\hline & \multicolumn{9}{|c|}{ ElementC3D20 } & \multicolumn{3}{c|}{ M+H } \\
\hline & $\begin{array}{l}\text { Tip } \\
\text { Displacement }\end{array}$ & $\begin{array}{l}\text { Tip } \\
\text { Displacement }\end{array}$ & $\begin{array}{l}\% \\
\text { Diff }\end{array}$ & Agree? & $\begin{array}{l}\text { Tip } \\
\text { Displacement }\end{array}$ & $\begin{array}{l}\% \\
\text { Diff }\end{array}$ & Agree? \\
\hline In-Plane & 0.076423 & 0.08734 & 12.50 & No & 0.076423 & 0.00 & Yes \\
\hline $\begin{array}{l}\text { Out-of- } \\
\text { Plane }\end{array}$ & 0.473575 & 0.5022 & 5.70 & No & 0.475081 & 0.32 & Yes \\
\hline
\end{tabular}

\begin{tabular}{|c|c|c|c|c|c|c|c|}
\hline \multicolumn{8}{|c|}{ Element C3D20R } \\
\hline & ABAQUS & \multicolumn{2}{|c|}{ Theoretical } & & \multicolumn{3}{|c|}{$\mathrm{M}+\mathrm{H}$} \\
\hline Load & $\begin{array}{l}\text { Tip } \\
\text { Displacement }\end{array}$ & $\begin{array}{l}\text { Tip } \\
\text { Displacement }\end{array}$ & $\begin{array}{l}\text { \% } \\
\text { Diff }\end{array}$ & Agree? & $\begin{array}{l}\text { Tip } \\
\text { Displacement }\end{array}$ & $\begin{array}{l}\% \\
\text { Diff }\end{array}$ & Agree? \\
\hline In-Plane & 0.087864 & 0.08734 & 0.60 & Yes & 0.088213 & 0.40 & Yes \\
\hline $\begin{array}{l}\text { Out-of- } \\
\text { Plane }\end{array}$ & 0.475081 & 0.5022 & 5.40 & No & 0.480103 & 1.05 & Yes \\
\hline
\end{tabular}

The model returned results that were consistent with the analytical results set out in [50]. ABAQUS passed the curved beam test. 


\subsubsection{Twisted Beam Test}

The curved beam test consisted of applying load to the free end of a twisted beam. The twisted beam was a $12.0 \times 1.1 \times 0.32$ beam subject to a $90^{\circ}$ twist along its length. The beam was assigned a mesh with a $12 \times 2 \times 1$ distribution. The beam geometry and element distribution is illustrated in Figure 3-4. The beam was constrained at one end and subjected to in-plane and out-of-plane unit loads at the free end. The beam was assigned a Young's modulus of $29.0 \times 10^{6}$ and a Poisson's ratio of 0.22 . The tip deflection resulting from the end loads are tabulated in Table 3-4.

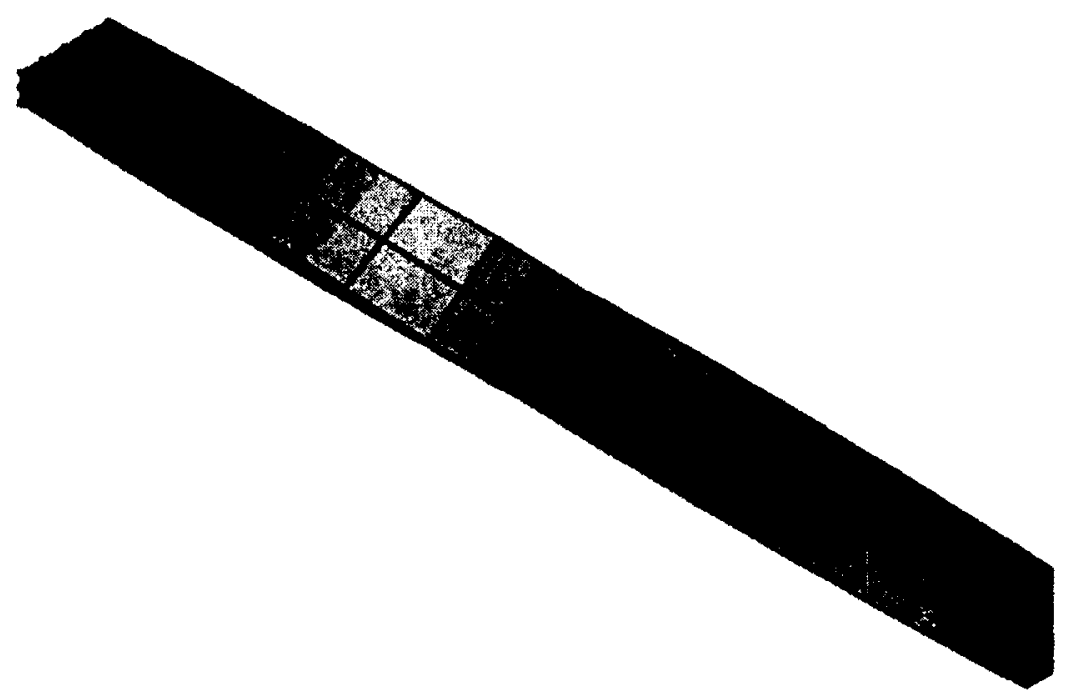

Figure 3-4: Twisted beam [50]

Table 3-4: Results for twisted beam geometry

\begin{tabular}{|c|c|c|c|c|c|c|c|}
\hline \multicolumn{8}{|c|}{ Element C3D20 } \\
\hline & ABAQUS & \multicolumn{3}{|c|}{ Theoretical } & \multicolumn{3}{|c|}{$\mathbf{M}+\mathbf{H}$} \\
\hline Load & $\begin{array}{l}\text { Tip } \\
\text { Displacement }\end{array}$ & $\begin{array}{l}\text { Tip } \\
\text { Displacement }\end{array}$ & $\begin{array}{l}\text { \% } \\
\text { Diff }\end{array}$ & Agree? & $\begin{array}{l}\text { Tip } \\
\text { Displacement }\end{array}$ & $\begin{array}{l}\% \\
\text { Diff }\end{array}$ & Agree? \\
\hline In-Plane & 0.005375 & 0.005424 & 0.90 & Yes & 0.005375 & 0.00 & Yes \\
\hline $\begin{array}{l}\text { Out-of- } \\
\text { Plane }\end{array}$ & 0.001747 & 0.001754 & 0.40 & Yes & 0.001745 & 0.11 & Yes \\
\hline
\end{tabular}




\begin{tabular}{|l|l|l|l|l|l|l|l|}
\hline \multicolumn{9}{|c|}{ Element C3D20R } \\
\hline & \multicolumn{1}{|c|}{ ABAQUS } & \multicolumn{3}{c|}{ Theoretical } & \multicolumn{3}{c|}{ M+H } \\
\hline Load & $\begin{array}{l}\text { Tip } \\
\text { Displacement }\end{array}$ & $\begin{array}{l}\text { Tip } \\
\text { Displacement }\end{array}$ & $\begin{array}{l}\text { \% } \\
\text { Diff }\end{array}$ & Agree? & $\begin{array}{l}\text { Tip } \\
\text { Displacement }\end{array}$ & $\begin{array}{l}\% \\
\text { Diff }\end{array}$ & Agree? \\
\hline In-Plane & 0.005386 & 0.005424 & 0.70 & Yes & 0.005386 & 0.00 & Yes \\
\hline $\begin{array}{l}\text { Out-of- } \\
\text { Plane }\end{array}$ & 0.001752 & 0.001754 & 0.11 & Yes & 0.001752 & 0.00 & Yes \\
\hline
\end{tabular}

The 20-node elastic element passed the twisted beam test. The model returned results that were consistent with the results set out in [50]. The model returned results that were fairly consistent with the theoretical results. ABAQUS passed the test.

\subsubsection{Rectangular Plate Test}

MacNeal and Harder proposed two geometries and two load cases for the rectangular plate test. The two plate geometries are illustrated in Figure 3-5. The first plate had dimensions of $2.0 \times 2.0 \times 0.01$ and an aspect ratio of 1.0. The second plate had dimensions of $10.0 \times 2.0 \times 0.01$ and an aspect ratio of 5.0. For both geometries, the plates were assigned a mesh with $8 \times 8 \times 1$ element distribution.

The first load case prescribed fully constrained plate edges and a point load of $4.0 \mathrm{x}$ $10^{-4}$ applied at the centre of the plate. The second load case prescribed simply supported plate edges and a pressure of $1.0 \times 10^{-4}$ applied to the entire surface of the plate.

Four tests were conducted. These tests consisted of a:

- Plate with an aspect ratio of 1.0 with clamped edges and a point load at the centre of the plate

- Plate with an aspect ratio of 1.0 with simply supported edges, subjected to a pressure load 
- Plate with an aspect ratio of 5.0 with clamped edges and a point load at the centre of the plate

- Plate with an aspect ratio of 5.0 with simply supported edges, subjected to a pressure load
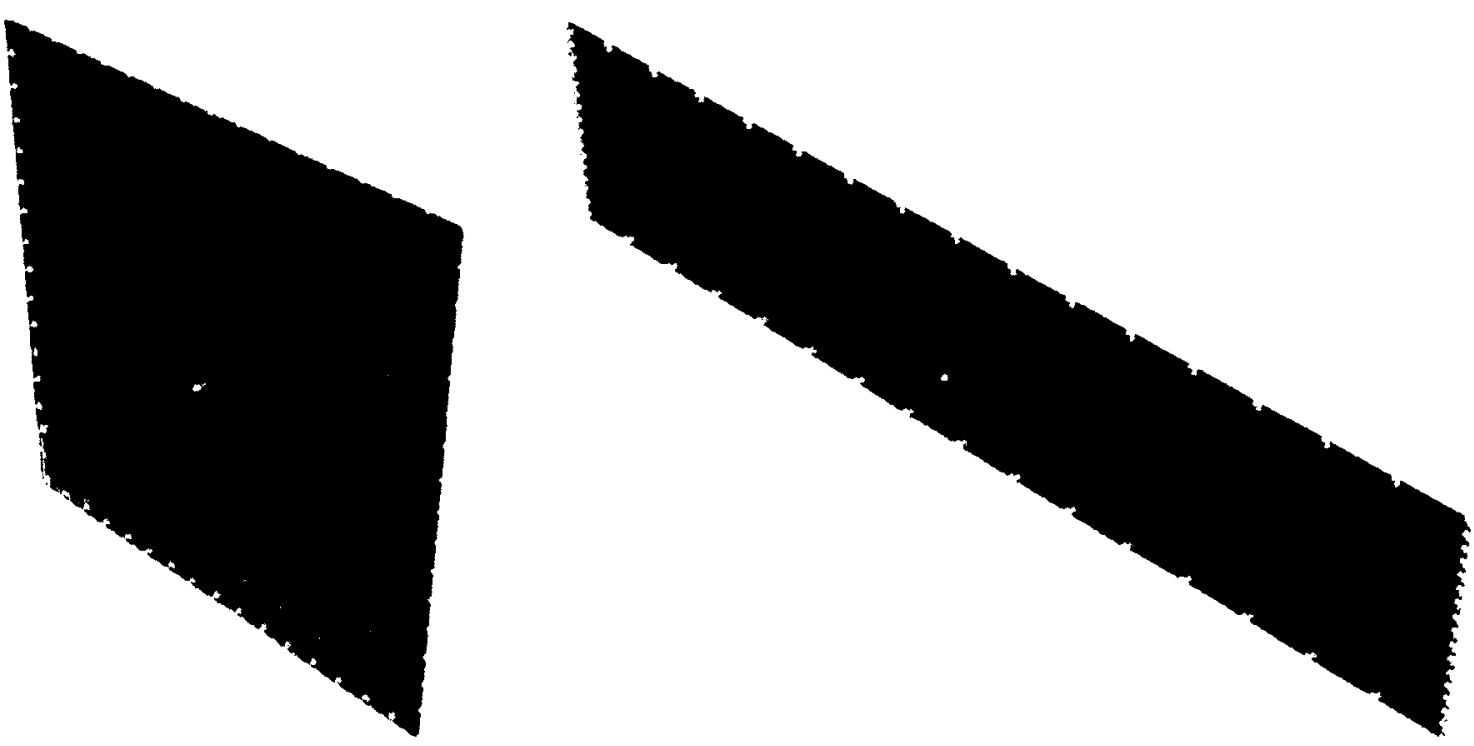

Figure 3-5: Plate geometry: (left) aspect ratio 1.0 and (right) aspect ratio 5.0 [50]

Table 3-5: Results for rectangular plate test

\begin{tabular}{|l|l|l|l|l|l|l|l|}
\hline & \multicolumn{9}{|c|}{ Aspect Ratio 1.0, Element C3D20 } \\
\hline & $\begin{array}{l}\text { Tip } \\
\text { Displacement }\end{array}$ & $\begin{array}{l}\text { Tip } \\
\text { Displacement }\end{array}$ & $\begin{array}{l}\text { \% } \\
\text { Diff }\end{array}$ & Agree? & $\begin{array}{l}\text { Tip } \\
\text { Displacement }\end{array}$ & $\begin{array}{l}\text { \% } \\
\text { Diff }\end{array}$ & Agree? \\
\hline & & & & & & \\
\hline $\begin{array}{l}\text { Clamped } \\
\text { edges, Point } \\
\text { load }\end{array}$ & $4.03 \mathrm{E}-06$ & $4.062 \mathrm{E}-06$ & 0.79 & Yes & $4.03 \mathrm{E}-06$ & 0.00 & Yes \\
\hline $\begin{array}{l}\text { Simply } \\
\text { supported } \\
\text { edges, } \\
\text { pressure load }\end{array}$ & $4.60 \mathrm{E}-06$ & $5.60 \mathrm{E}-06$ & 17.86 & No & $4.60 \mathrm{E}-06$ & 0.00 & Yes \\
\hline
\end{tabular}




\begin{tabular}{|l|l|l|l|l|l|l|l|}
\hline \multicolumn{9}{|c|}{ Aspect Ratio 1.0, Element C3D20R } \\
\hline & ABAQUS & \multicolumn{3}{|c|}{ Theoretical } & \multicolumn{3}{c|}{ M+H } \\
\hline Load & $\begin{array}{l}\text { Tip } \\
\text { Displacement }\end{array}$ & $\begin{array}{l}\text { Tip } \\
\text { Displacement }\end{array}$ & $\begin{array}{l}\text { \% } \\
\text { Diff }\end{array}$ & Agree? & $\begin{array}{l}\text { Tip } \\
\text { Displacement }\end{array}$ & $\begin{array}{l}\% \\
\text { Diff }\end{array}$ & Agree? \\
\hline $\begin{array}{l}\text { Clamped } \\
\text { edges, Point } \\
\text { load }\end{array}$ & $4.09 \mathrm{E}-06$ & $4.062 \mathrm{E}-06$ & 0.69 & Yes & $4.09 \mathrm{E}-06$ & 0.00 & Yes \\
\hline $\begin{array}{l}\text { Simply } \\
\text { supported } \\
\text { edges, } \\
\text { pressure load }\end{array}$ & $5.28 \mathrm{E}-06$ & $5.60 \mathrm{E}-06$ & 5.71 & No & $5.29 \mathrm{E}-06$ & 0.19 & Yes \\
\hline
\end{tabular}

\begin{tabular}{|l|l|l|l|l|l|l|l|}
\hline \multicolumn{8}{|c|}{ Aspect Ratio 5.0, Element C3D20 } \\
\hline & ABAQUS & \multicolumn{3}{|c|}{ Theoretical } & \multicolumn{3}{c|}{ M+H } \\
\hline Load & $\begin{array}{l}\text { Tip } \\
\text { Displacement }\end{array}$ & $\begin{array}{l}\text { Tip } \\
\text { Displacement }\end{array}$ & $\begin{array}{l}\% \\
\text { Diff }\end{array}$ & Agree? & $\begin{array}{l}\text { Tip } \\
\text { Displacement }\end{array}$ & $\begin{array}{l}\% \\
\text { Diff }\end{array}$ & Agree? \\
\hline $\begin{array}{l}\text { Clamped } \\
\text { edges, Point } \\
\text { load }\end{array}$ & $1.33 E-05$ & $1.297 \mathrm{E}-05$ & 2.54 & Yes & $1.33 \mathrm{E}-05$ & 0.00 & Yes \\
\hline $\begin{array}{l}\text { Simply } \\
\text { supported } \\
\text { edges, } \\
\text { pressure } \\
\text { load }\end{array}$ & & & & & & & \\
\hline
\end{tabular}

\begin{tabular}{|l|l|l|l|l|l|l|l|}
\hline & \multicolumn{6}{|c|}{ Aspect Ratio 5.0, Element C3D20R } \\
\hline & ABAQUS & \multicolumn{2}{|c|}{ Theoretical } & \multicolumn{3}{|c|}{} \\
\hline & $\begin{array}{l}\text { Tip } \\
\text { Displacement }\end{array}$ & $\begin{array}{l}\text { Tip } \\
\text { Displacement }\end{array}$ & $\begin{array}{l}\% \\
\text { Diff }\end{array}$ & Agree? & $\begin{array}{l}\text { Tip } \\
\text { Displacement }\end{array}$ & $\begin{array}{l}\text { \% } \\
\text { Diff }\end{array}$ & Agree? \\
\hline $\begin{array}{l}\text { Load } \\
\text { Clamped } \\
\text { edges, Point } \\
\text { load }\end{array}$ & $1.30 \mathrm{E}-05$ & $1.297 \mathrm{E}-05$ & 0.23 & Yes & $1.31 \mathrm{E}-05$ & 0.76 & Yes \\
\hline $\begin{array}{l}\text { Simply } \\
\text { supported } \\
\text { edges, } \\
\text { pressure load }\end{array}$ & $6.27 \mathrm{E}-06$ & $7.23 \mathrm{E}-06$ & 13.28 & No & $6.27 \mathrm{E}-06$ & 0.00 & Yes \\
\hline
\end{tabular}


In most cases, $\mathrm{ABAQUS}$ provided results that were consistent with the theoretical results set out in [50]. In the cases where ABAQUS returned results that were significantly different from theoretical results, the ABAQUS results were consistent with results obtained by MacNeal and Harder [50]. The results obtained by MacNeal and Harder were the most accurate obtainable by a finite element model with such sparse meshing. ABAQUS passed the flat plate test.

In the case of the test involving the pressure loaded, simply supported, aspect ratio 5.0 plate with C3D20 elements, the finite element analysis returned results that were inconsistent with those set out in [50]. In this case, both MacNeal and Harder [50] and the finite element analysis returned results that were consistently inconsistent with the theoretical results.

\subsubsection{Scordelis-Lo Roof test}

The Scordelis-Lo roof consisted of a curved plane. The curved plane had a radius of curvature of 25.0, a length of 50.0, a thickness of 0.25 and an arc length of 80 degrees. The plane was assigned a Young's modulus of $\mathrm{E}=4.32 \times 10^{8}$ and a Poisson's ratio of $\mu=$ 0.0. The Scordelis-Lo roof is illustrated in Figure 3-6.

The plane was loaded to 90 per unit area in the negative $Z$ direction. The curved edges of the plane were constrained in place. The Z-component of displacement of the plane edges is tabulated and compared to expected values in Table 3-6. 


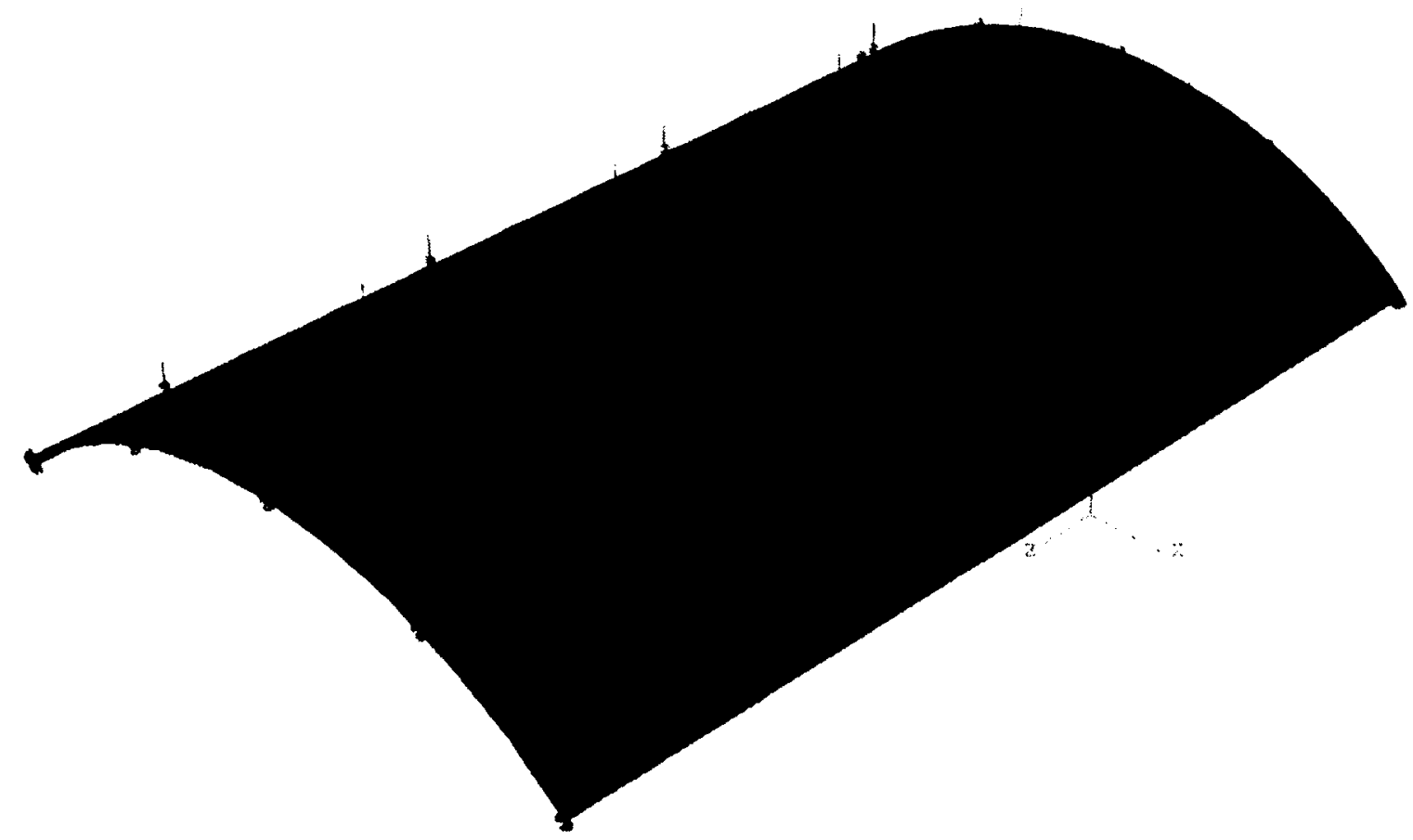

Figure 3-6: Scordelis-Lo roof [50]

Table 3-6: Results for Scordelis-Lo roof test

\begin{tabular}{|l|l|l|l|l|l|l|l|}
\hline & ABAQUS & \multicolumn{2}{|l|}{ Theoretical } & \multicolumn{2}{l|}{ M+H } \\
& & & & & & \\
& Displacement & Displacement & $\%$ Diff & Agree? & Displacement & $\%$ Diff & Agree? \\
\hline C3D20 & $2.519 E-01$ & $3.024 E-01$ & 16.70 & No & $2.455 E-01$ & 2.59 & Yes \\
\hline C3D20R & $3.086 E-01$ & $3.024 E-01$ & 2.05 & Yes & $3.021 E-01$ & 2.15 & Yes \\
\hline
\end{tabular}

ABAQUS produced results that were inconsistent with the theoretical results. However, ABAQUS did produce results that were consistent with those obtained by MacNeal and Harder $(\mathrm{M}+\mathrm{H})$. ABAQUS passed the Scordelis-Lo roof test.

\subsection{Geometric Nonlinear Tests}

Validation of the modeler's ability to implement geometric nonlinear problems was verified by undertaking geometric nonlinear benchmark tests. The first test consisted of a 
heavily loaded flat beam. The second test consisted of a bar loaded both axially and transversely.

In each benchmark test, geometry and loading condition is supplied [42, 51]. The theoretical solution is also supplied [42, 51]. The ABAQUS model is considered to have succeeded if it returned results that agreed with the theoretical solutions.

\subsubsection{Heavily Loaded Flat Beam Test}

The test consisted of a flat beam subjected to a consistently oriented shear load applied to the end face of the beam.

The plate had dimensions of $10.0 \times 1.0 \times 0.1 \mathrm{~m}$. The beam was assigned a mesh that was segmented into 8 and 16 elements along its length. The beam was assigned a Young's modulus of $E=1.2 \times 10^{6} \mathrm{~N} / \mathrm{m}^{2}$ and a Poisson's ratio of 0.0 .

One end of the beam was fully constrained. The other end of the beam was subjected to a constantly oriented out-of-plane shear stress. The stress ranged in magnitude from 0 $\mathrm{N} / \mathrm{m}^{2}$ to $40 \mathrm{~N} / \mathrm{m}^{2}$.

The vertical and horizontal components of tip displacement were measured and compared to the solution presented in [51]. The finite element model of the beam (with 16 elements) is presented in Figure 3-7. The results are presented in Figure 3-8. 


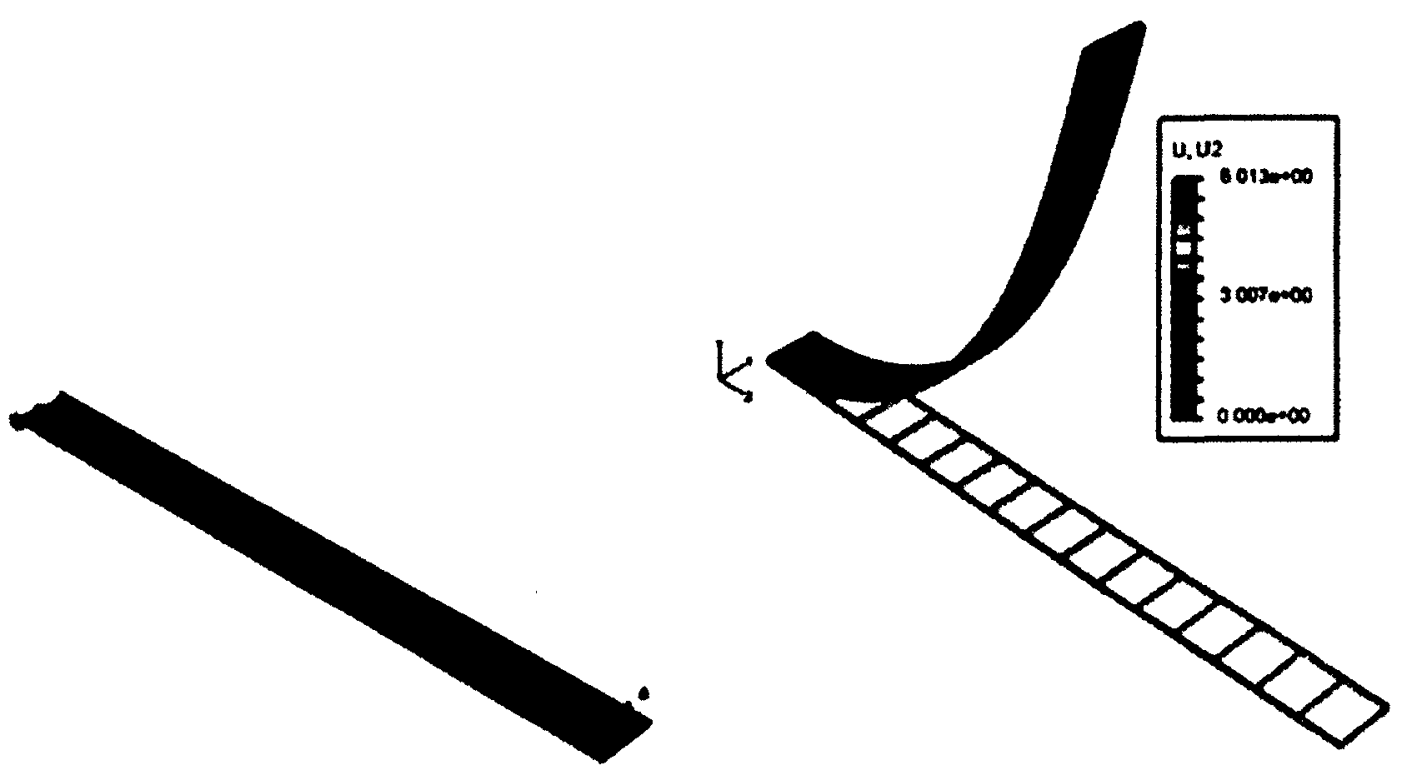

Figure 3-7: Flat beam with 16 elements (left) and displacement resulting from load (right)

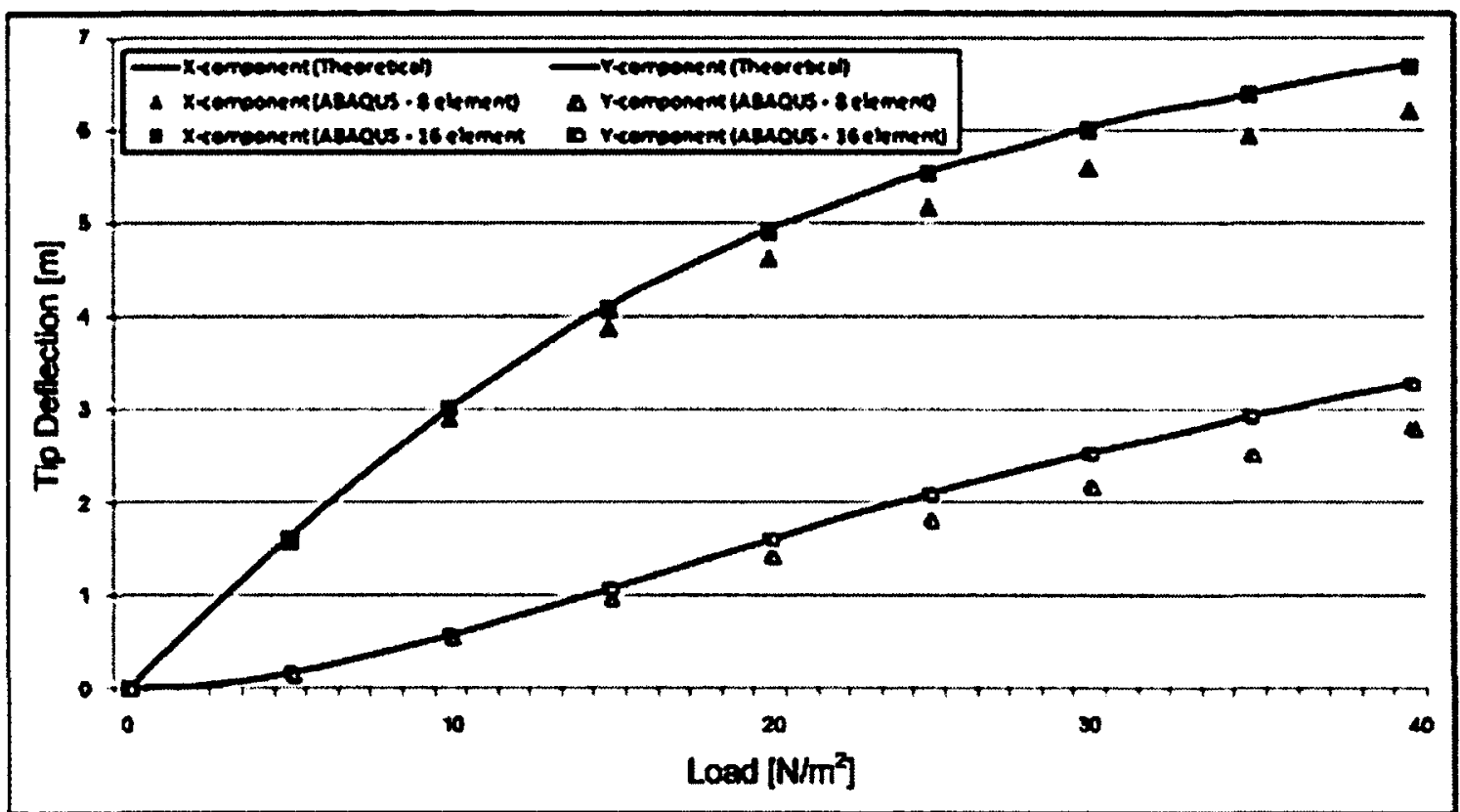

Figure 3-8: Results for heavily loaded flat plate: vertical (y) and horizontal (x) components of tip displacement 
ABAQUS returned results that were consistent with those provided in [51]. The finite flat beam with 16 elements along its length provided more accurate results than the model with only eight elements along its length.

\subsubsection{Dually-Loaded Beam Test}

This benchmark test consisted of a beam loaded in both the axial and transverse direction. The beam had dimensions of $50.0 \times 1.0 \times 1.0 \mathrm{~m}$. The beam was assigned a mesh with a distribution of $200(50 \times 2 \times 2)$ elements.

One end of the beam was fixed in space but was allowed to rotate in the direction of the applied transverse load. The beam was loaded with an axial point load and a transverse pressure load. The axial load was set to $1000 \mathrm{~N}$. The transverse pressure load varied logarithmically $\left(10^{3}, 10^{4}, 10^{5} \mathrm{~N} / \mathrm{m}^{2}\right)$. The theoretical solution giving beam tip displacement was given in [42]. The exercise was undertaken once while considering the geometric nonlinear effects (NLGeom on) and again while ignoring geometric nonlinear effects (NLGeom off). The beam is presented in Figure 3-9. The results are presented in Table 3-7.

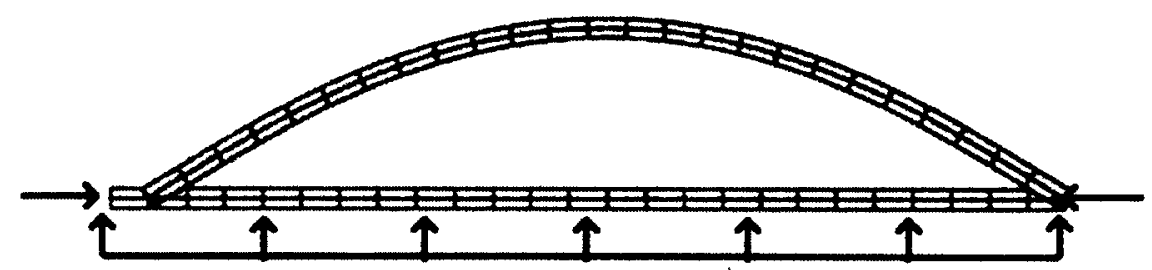

Figure 3-9: Dually-loaded beam 
Table 3-7: Results for dually-loaded beam test

\begin{tabular}{|c|c|c|c|c|c|c|}
\hline & & & \multicolumn{2}{|c|}{ NLGeom Off } & \multicolumn{2}{c|}{ NLGeom ON } \\
\hline $\begin{array}{c}\text { Axial } \\
\text { load (N) }\end{array}$ & $\begin{array}{c}\text { Lateral load } \\
\left(N / \mathrm{m}^{2}\right)\end{array}$ & $\begin{array}{c}\text { Theoretical } \\
\text { deflection }(\mathrm{m})\end{array}$ & $\begin{array}{c}\text { Deflection } \\
(\mathrm{m})\end{array}$ & $\begin{array}{c}\text { Deflection Diff } \\
(\mathrm{m})\end{array}$ & $\begin{array}{c}\% \\
\text { Diff }\end{array}$ \\
\hline 1000 & $1.00 \mathrm{E}+02$ & $4.90 \mathrm{E}-02$ & $4.90 \mathrm{E}-02$ & $0.10 \%$ & $4.89 \mathrm{E}-02$ & $0.22 \%$ \\
\hline 1000 & $2.50 \mathrm{E}+02$ & $1.23 \mathrm{E}-01$ & $1.22 \mathrm{E}-01$ & $0.49 \%$ & $1.22 \mathrm{E}-01$ & $0.65 \%$ \\
\hline 1000 & $5.00 \mathrm{E}+02$ & $2.45 \mathrm{E}-01$ & $2.48 \mathrm{E}-01$ & $1.10 \%$ & $2.45 \mathrm{E}-01$ & $0.20 \%$ \\
\hline 1000 & $7.50 \mathrm{E}+02$ & $3.68 \mathrm{E}-01$ & $3.67 \mathrm{E}-01$ & $0.30 \%$ & $3.67 \mathrm{E}-01$ & $0.35 \%$ \\
\hline 1000 & $1.00 \mathrm{E}+03$ & $4.89 \mathrm{E}-01$ & $4.89 \mathrm{E}-01$ & $0.02 \%$ & $4.89 \mathrm{E}-01$ & $0.02 \%$ \\
\hline 1000 & $2.50 \mathrm{E}+03$ & $1.23 \mathrm{E}+00$ & $1.22 \mathrm{E}+00$ & $0.90 \%$ & $1.22 \mathrm{E}+00$ & $0.33 \%$ \\
\hline 1000 & $5.00 \mathrm{E}+03$ & $2.45 \mathrm{E}+00$ & $2.38 \mathrm{E}+00$ & $2.81 \%$ & $2.45 \mathrm{E}+00$ & $0.33 \%$ \\
\hline 1000 & $7.50 \mathrm{E}+03$ & $3.68 \mathrm{E}+00$ & $3.48 \mathrm{E}+00$ & $5.52 \%$ & $3.67 \mathrm{E}+00$ & $0.33 \%$ \\
\hline 1000 & $1.00 \mathrm{E}+04$ & $4.89 \mathrm{E}+00$ & $4.47 \mathrm{E}+00$ & $8.53 \%$ & $4.89 \mathrm{E}+00$ & $0.02 \%$ \\
\hline 1000 & $2.50 \mathrm{E}+04$ & $1.23 \mathrm{E}+01$ & $8.78 \mathrm{E}+00$ & $28.38 \%$ & $1.22 \mathrm{E}+01$ & $0.33 \%$ \\
\hline 1000 & $5.00 \mathrm{E}+04$ & $2.45 \mathrm{E}+01$ & Failed* & N/A & $2.45 \mathrm{E}+01$ & $0.31 \%$ \\
\hline
\end{tabular}

The finite element model of the beam that considered the geometric nonlinearity effect successfully returned results that were consistent with the theoretically determined solution. The finite element model that did not consider the geometric nonlinearity effect returned results that diverged greatly from the mathematically determined solution when larger loads were applied.

\subsection{Piezoelectric Tests}

Validation of the modeler's ability to implement piezoelectric problems was verified by undertaking piezoelectric tests. The first piezoelectric tests consisted of simple, mathematically determinable problems furnished in [44]. Other piezoelectric tests consisted of reproducing experimental results published in [4] 
The piezoelectric problems undertaken were:

- Piezoelectrically-Extended Straight Beam Test

- Piezoelectrically Sheared Straight Beam Test

- Piezoelectrically-Actuated Bimorphic Beam Test

- Piezoelectric hollow active fibre

- Piezoelectric Hollow Active Fibre Composite (HAFC)

The experimental problems undertaken in [4] were the actuation of a hollow active fibre and the actuation of a hollow active fibre composite.

\subsubsection{Piezoelectrically-Extended Straight Beam Test}

The Piezoelectrically-Extended Straight Beam test consisted of the simulation of extension of a beam due to the piezoelectric effect. The beam geometry for these tests was the same as those in defined section 3.1.2. The beam was assigned a mesh that was subdivided into six elements with (a) rectangular, (b) parallelogram and (c) trapezoidal geometry. The piezoelectric material used (PZT-5A) was assigned Young's moduli of $\mathrm{E}_{11}$ $=\mathrm{E}_{22}=60.6 \times 10^{9} \mathrm{~N} / \mathrm{m}^{2}$ and $\mathrm{E}_{33}=48.3 \times 10^{9} \mathrm{~N} / \mathrm{m}^{2}$, Poisson's ratios of $\mu_{12}=0.2896$ and $\mu$ ${ }_{13}=\mu_{23}=0.408$, shear moduli of $G_{12}=23.6 \times 10^{9} \mathrm{~N} / \mathrm{m}^{2}$ and $G_{13}=G_{23}=2.29 \times 10^{9} \mathrm{~N} / \mathrm{m}^{2}$, permittivities of $\mathrm{k}_{11}=\mathrm{k}_{22}=2.77 \times 10^{-8} \mathrm{~F} / \mathrm{m}$ and $\mathrm{k}_{33}=3.01 \times 10^{-8} \mathrm{~F} / \mathrm{m}$ and piezoelectric coefficients of $d_{15}=d_{24}=741 \times 10^{-12} \mathrm{C} / \mathrm{N}, d_{31}=d_{32}=-274 \times 10^{-12} \mathrm{C} / \mathrm{N}$ and $d_{33}=593 \times$ $10^{-12} \mathrm{C} / \mathrm{N}$. The beam was constrained at one end and a potential difference of $1 \mathrm{~V}$ (or an electric field intensity of $0.01 \mathrm{~V} / \mathrm{mm}$ ) was established through the thickness of the beam. 
The displacement of the free end was measured. A schematic of the setup is illustrated in Figure 3-10. The results are tabulated in Table 3-8.

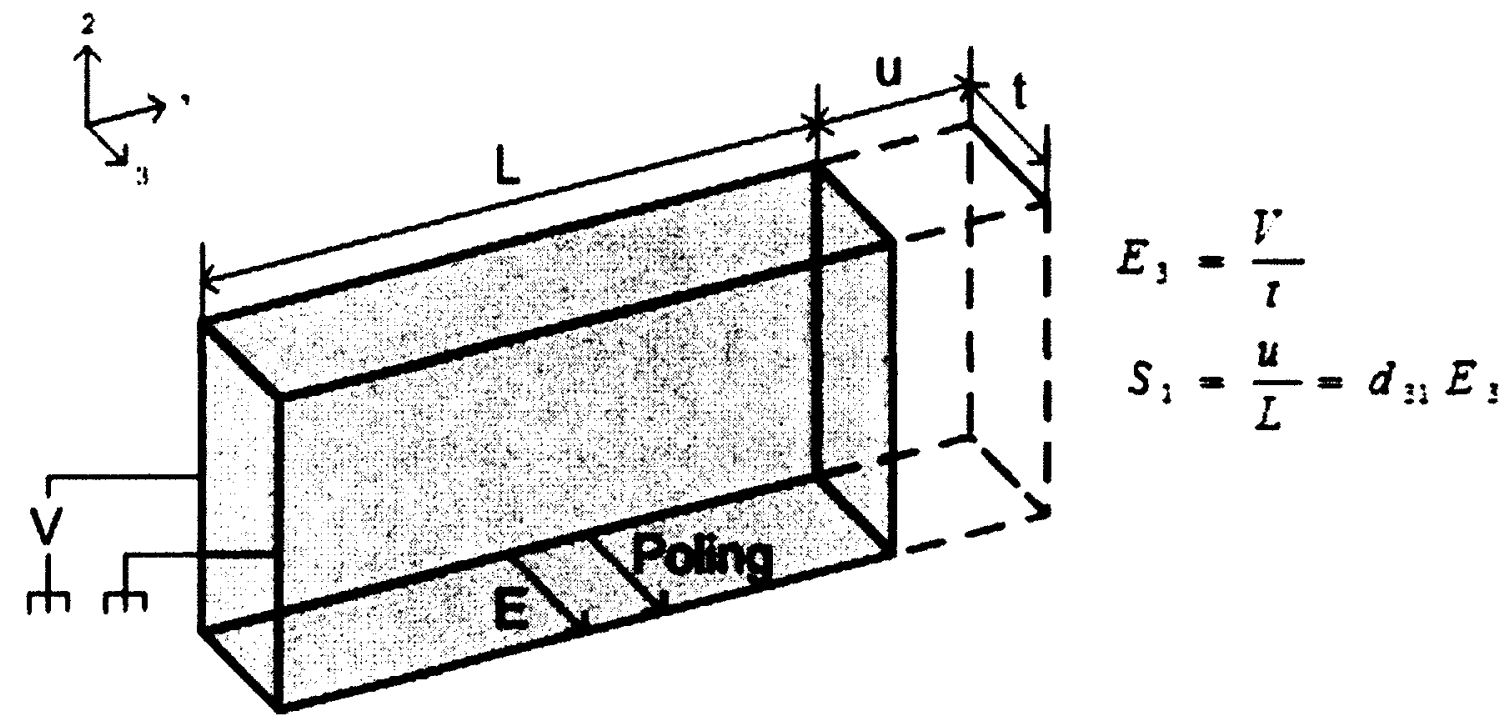

Figure 3-10: Straight beam undergoing piezoelectric extension [44]

Table 3-8: results for piezoelectrically-extended straight beam test

\begin{tabular}{|l|l|l|l|}
\hline & \multicolumn{3}{|c|}{ Displacement, u (m) } \\
\hline & Theoretical & ABAQUS & $\%$ Diff \\
\hline Rectangular Elements (Type a) & $1.644 \mathrm{E}-08$ & $1.628 \mathrm{E}-08$ & 0.973 \\
\hline Parallelogram Elements (Type b) & $1.644 \mathrm{E}-08$ & $1.630 \mathrm{E}-08$ & 0.859 \\
\hline Trapezoidal Elements (Type c) & $1.644 \mathrm{E}-08$ & $1.635 \mathrm{E}-08$ & 0.576 \\
\hline
\end{tabular}

The finite element analysis returned tip displacement results that were consistent with the theoretical tip displacement. The Piezoelectrically-extended straight beam test was passed successfully.

\subsubsection{Piezoelectrically-Sheared Straight Beam Test}

The piezoelectrically sheared straight beam test consists of the simulation of the shearing of a beam due to the piezoelectric effect. The beam was assigned geometry used for the 
straight beam test in section 3.1.2. The beam was subdivided into six elements with (a) rectangular, (b) parallelogram and (c) trapezoidal geometry. The Piezoelectric material used (PZT-5A) was assigned material properties described in section 3.3.1. The beam was constrained at one end and a potential difference of $1 \mathrm{~V}$ (or an electric field intensity of $0.01 \mathrm{~V} / \mathrm{mm}$ ) was established through the thickness of the beam. The displacement of the free end was measured. A schematic of the setup is illustrated in Figure 3-11. The results are tabulated in Table 3-9.

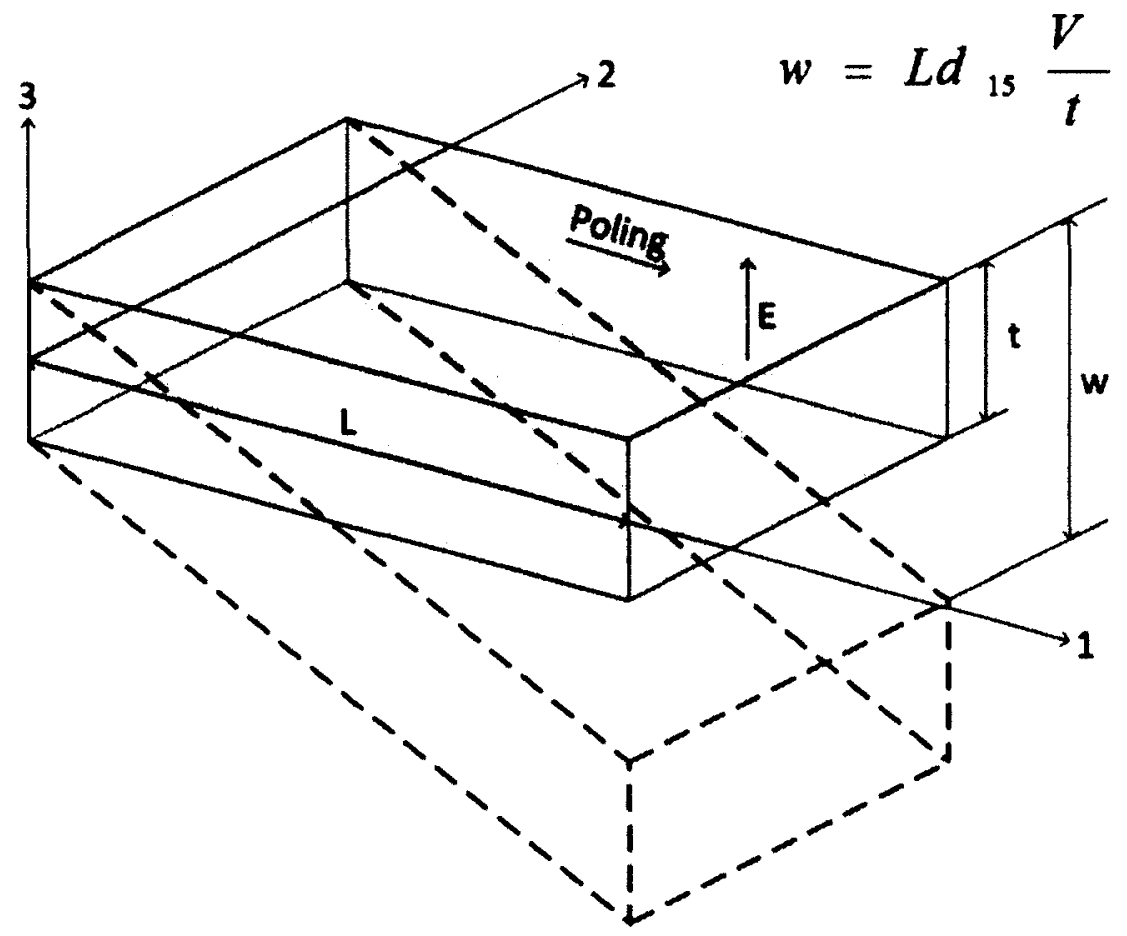

Figure 3-11: Straight beam undergoing piezoelectric shear [44]

Table 3-9: Results for piezoelectric sheared straight beam test

\begin{tabular}{|l|l|l|l|}
\hline & \multicolumn{3}{|c|}{ Displacement, w (m) } \\
\hline & Theoretical & ABAQUS & $\%$ Diff \\
\hline Rectangular Elements (Type a) & $4.446 \mathrm{E}-08$ & $4.446 \mathrm{E}-08$ & 0.000 \\
\hline Parallelogram Elements (Type b) & $4.446 \mathrm{E}-08$ & $4.446 \mathrm{E}-08$ & 0.000 \\
\hline Trapezoidal Elements (Type c) & $4.446 \mathrm{E}-08$ & $4.446 \mathrm{E}-08$ & 0.000 \\
\hline
\end{tabular}


As seen in table 3-9, the finite element analysis returned tip displacement results that were consistent with the theoretical tip displacement. The Piezoelectrically sheared straight beam test was passed successfully.

\subsubsection{Piezoelectrically-Actuated Bimorphic Beam Test}

The piezoelectrically-actuated bimorphic beam test consists of the simulation of the deformation of a bimorphic beam due to the piezoelectric effect. The beam used had the geometry used for the straight beam test in section 3.2.2. The beam was subdivided into six elements with (a) rectangular, (b) parallelogram and (c) trapezoidal geometry. The elements were then subdivided again through the thickness of the plate. The piezoelectric material used (PZT-5A) was assigned material properties recorded in section 3.4.1. The beam was constrained at one end and a potential difference of $1 \mathrm{~V}$ (or an electric field intensity of $0.01 \mathrm{~V} / \mathrm{mm}$ ) was established through the thickness of the beam. The displacement of the free end was measured. A schematic of the setup is illustrated in Figure 3-12. The results are tabulated in Table 3-10. 


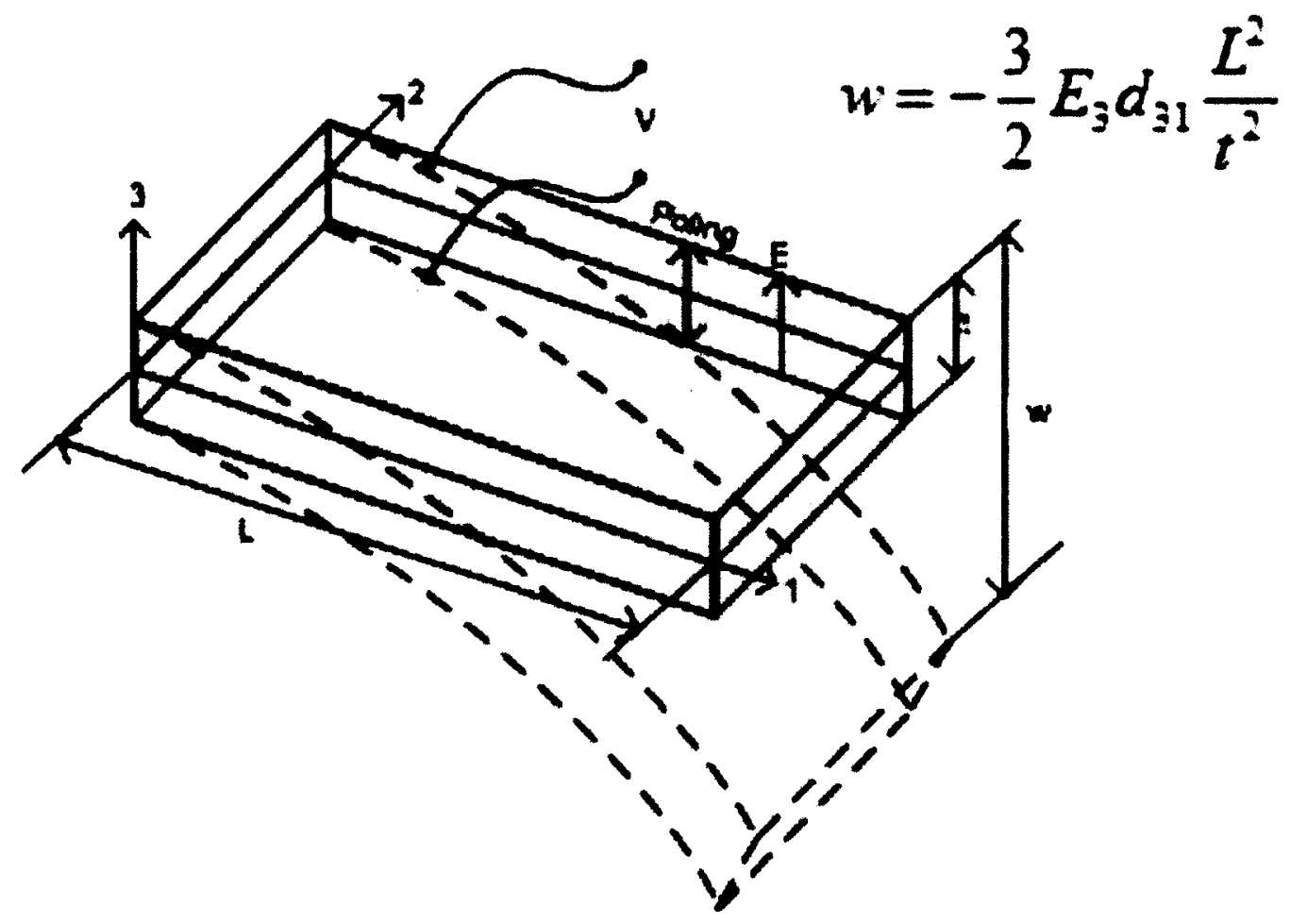

Figure 3-12: bimorphic beam undergoing piezoelectric deformation [44]

Table 3-10: Results for piezoelectrically-actuated bimorphic beam test

\begin{tabular}{|l|l|l|l|}
\hline & \multicolumn{3}{|c|}{ Displacement, w (m) } \\
\hline & Theoretical & ABAQUS & $\%$ Diff \\
\hline Rectangular Elements (Type a) & $1.4796 \mathrm{E}-08$ & $1.465 \mathrm{E}-08$ & 0.947 \\
\hline Parallelogram Elements (Type b) & $1.4796 \mathrm{E}-08$ & $1.465 \mathrm{E}-08$ & 0.947 \\
\hline Trapezoidal Elements (Type c) & $1.4796 \mathrm{E}-08$ & $1.465 \mathrm{E}-08$ & 0.947 \\
\hline
\end{tabular}

As seen, the finite element analysis returned tip displacement results that were consistent with the theoretical tip displacement. The piezoelectrically-actuated bimorphic beam test was passed successfully. 


\subsubsection{Piezoelectric Hollow Active Fibre test}

Experiments have been conducted in order to determine the actuation performance of hollow piezoelectric fibres embedded in an epoxy matrix. The results of these experiments were reported in [4].

Finite element analyses were conducted in order to validate the ability of the analyst to produce successful analyses using the piezoelectric element C3D20E. Finite element analyses were created, reproducing the geometry and conditions of the hollow active fibres and the hollow active fibre composites (HAFCs) used in [4].

One of the Hollow Active Fibres presented in [4] had an aspect ratio (ratio of wall thickness to outer radius) of 0.42 , an outer wall radius of $450 \mu \mathrm{m}$ and wall thickness of $190 \mu \mathrm{m}$. The piezoelectric material used (PZT-5A) was assigned material properties recorded in section 3.4.1. Application of an electric potential difference of $57 \mathrm{~V}$ between the inner and outer walls of the fibre generated an electric field intensity of $300 \mathrm{~V} / \mathrm{mm}$. The fibre in [4] produced a longitudinal strain of 80 microstrain.

A finite element model was created, reproducing the hollow fibre in [4]. The quarterfibre finite element model had a length of $77 \mathrm{~mm}$. When actuated, the fibre extended 6.15 $\mu \mathrm{m}$, resulting in an average strain of 79.2 microstrain. The actuated fibre model is illustrated in Figure 3-13. The finite element analysis returned results that were consistent with those published in [4]. The finite element analysis succeeded in reproducing the piezoelectric fibre experiment. 


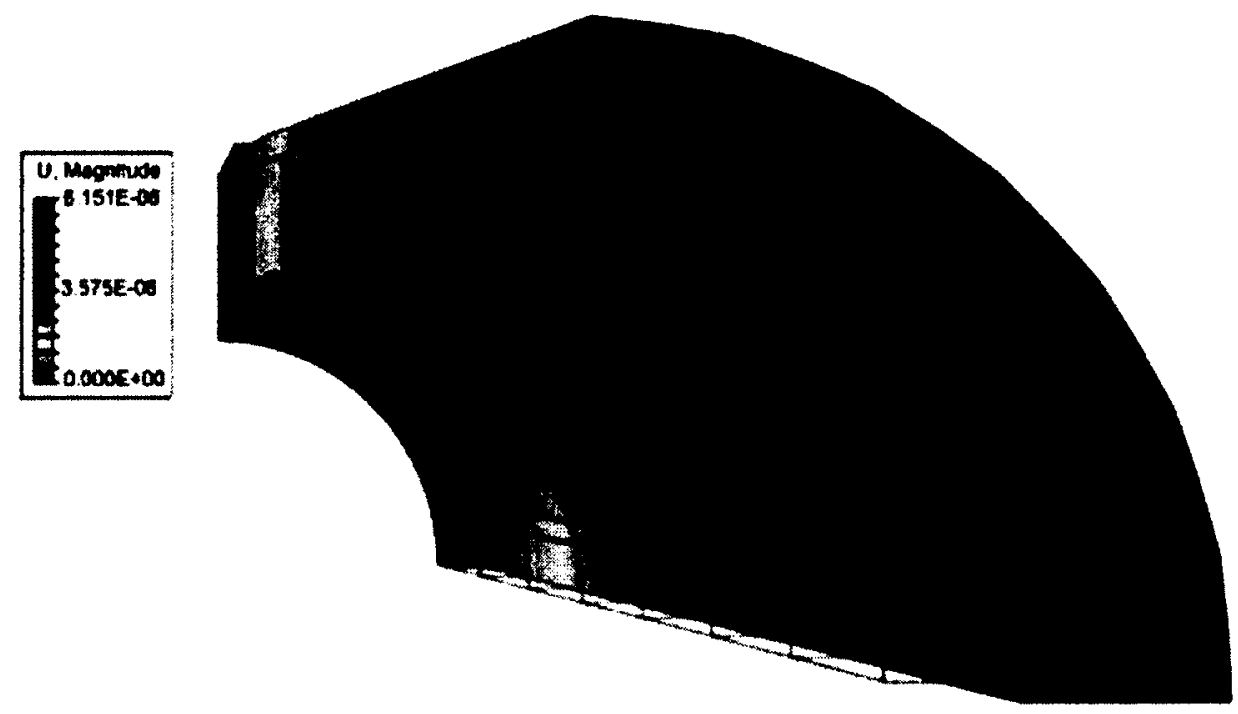

Figure 3-13: Actuated quarter-fibre

\subsubsection{Piezoelectric Hollow Active Fibre Composite (HAFC) Test}

Experiments were also conducted on Active Fibre Composites (AFCs) in [4]. These AFCs consisted of the hollow piezoelectric fibre embedded in an epoxy matrix material. The fibre was the same 0.42 aspect ratio used previously. The matrix was applied such that the composite had a fibre volume fraction of $4.6 \%$. The same actuating potential of $57 \mathrm{~V}(300 \mathrm{~V} / \mathrm{mm})$ was applied. The composite produced an average strain of approximately 45 microstrain.

The matrix material was Insulcast $501 /$ Insulcure 24. The matrix material had a Young's Modulus of $\mathrm{E}=1.29 \times 10^{9} \mathrm{~Pa}$ and a Poisson's ratio of $\mu=0.3$.

The hollow active fibre composite consisted of the same hollow piezoelectric fibre embedded in an epoxy matrix. A model of a quarter fibre was created. The hollow fibre had a wall thickness of $190 \mu \mathrm{m}$ and an external radius of $450 \mu \mathrm{m}$. The fibre was embedded in an epoxy matrix such that the fibre-to-matrix volume fraction was $4.6 \%$. 
This corresponded to having a quarter fibre with an external diameter of $450 \mu \mathrm{m}$ embedded in an epoxy matrix measuring $1685 \mu \mathrm{m}$ by $1400 \mu \mathrm{m}$. The entire HAFC was 77 mm long. A quarter cell of the HAFC is illustrated in Figure 3-14.

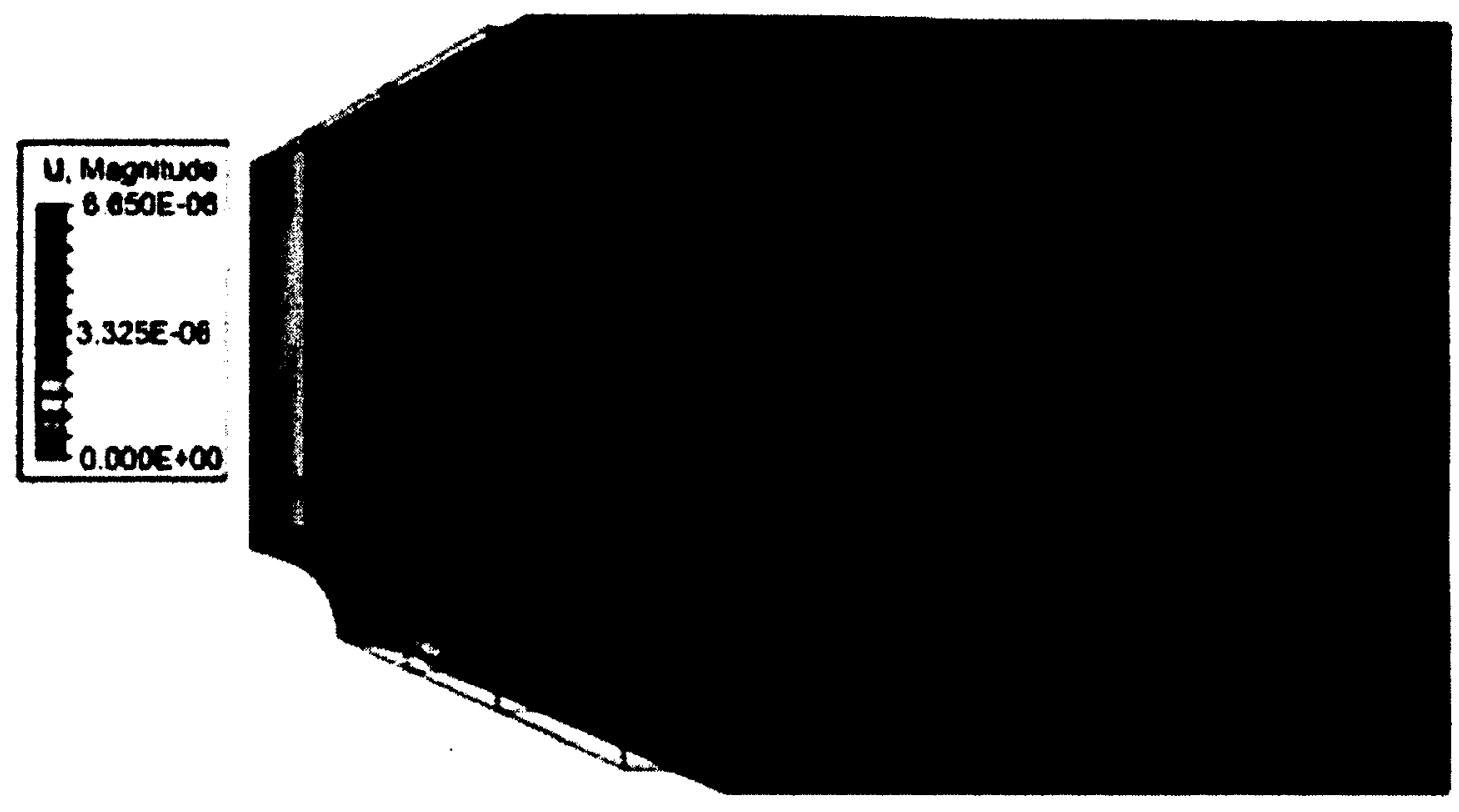

Figure 3-14: One quarter of a unit cell of the hollow active fibre composite

The finite element model of the HAFC experienced a longitudinal strain of $3.69 \mu \mathrm{m}$, resulting in an average strain of 48 microstrain, which was consistent with [4]. The finite element model successfully reproduced the experimental HAFC.

\subsection{Conclusions}

Tests were created to gauge the capabilities of the finite element analysis software as well as the capabilities of the finite element analyst. These tests included linear elastic tests, geometric nonlinear tests and piezoelectric tests. The modeler created finite element models that successfully returned the expected solutions to each of the test cases. 


\section{Chapter 4: Analysis and Results}

\subsection{Introduction}

A piezoceramic bimorph actuator was studied. The reliability of Hollow Active Fibre Composites to damage was also considered as a part of this study. The piezoceramic bimorph actuator was designed to create a morphing wing for a fixed-wing aircraft. The morphing capability was used to replace conventional ailerons. A schematic of the wing is presented in Figure 4-1. The morphing wing was developed for a micro, unmanned air vehicle (MAV) that is capable of performing the prop-hanging maneuver. The prophanging maneuver is a high angle of attack flight configuration where the aircraft is essentially hovering. Hover is a desirable capability for MAVs on missions such as reconnaissance or inspection. The lifting force holding the aircraft aloft is provided exclusively by the propulsion system (i.e. thrust is greater than weight.)

The prop-hanging maneuver requires powerful roll control. In order to provide such roll control, the MAV wing must be capable of large aileron deflection. A piezoceramic bimorph actuator-based morphing wing would be capable of large deflection of the part of the morphing wing which is replacing the aileron. The deflection ability of the piezoceramic bimorph actuator was the subject of study. 
The prop-hanging maneuver also requires an experienced pilot. The piezoceramic bimorph actuator relies on an active feedback control system for operation. A control system could operate the piezoceramic bimorph wing while reducing the need for pilot experience.

Furthermore, the MAV would have very tight design envelopes for size, structural weight and power consumption. The piezoceramic bimorph actuator has excellent specific power and low energy consumption.

The piezoelectric material-based morphing wing would consist of a series of Macro Fibre Composite (MFC)-actuated or Hollow Active Fibre Composite (HAFC)-actuated bimorphic structures. These bimorphic structures would deliver the desired aileron deflection required to perform the prop-hanging maneuver. Piezoelectric material-based morphing wing is illustrated in Figure 4-1.

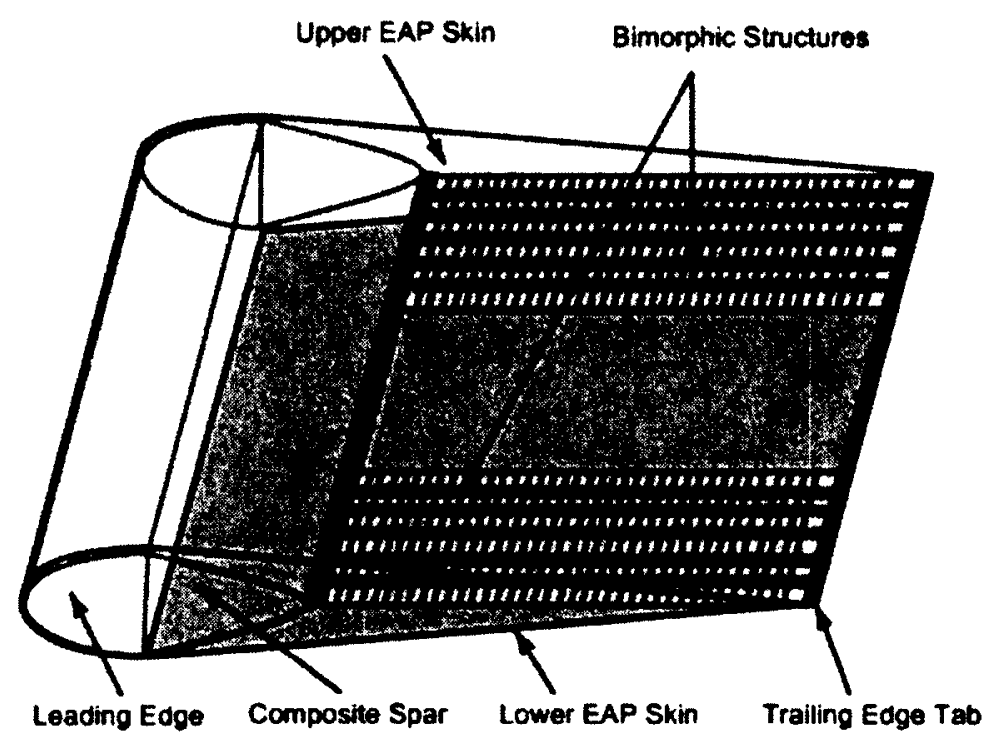

Figure 4-1: Macro Fibre Composite (MFC)-based morphing wing [54] 
It is hypothesized that the aileron deflection performance of the bimorphic structures could be further amplified with the application of an axial precompression force [2]. Such an axial precompression force could be delivered by Electro-Active Polymer (EAP) skin attached to the bimorphic structures, delivering a controlled precompressive force.

The goal of this thesis is to determine and compare the aileron tip displacement performance of an axially-precompressed, Macro Fibre Composite (MFC)-actuated and Hollow Active Fibre Composite (HAFC)-actuated bimorphic structures. A physical MFC-actuated bimorphic structure was manufactured. A finite element model of this MFC-actuated bimorphic structure was also created. The finite element model of the MFC-actuated bimorphic structure was used to examine the effects of load conditions not achievable by the physical actuator. A finite element model of the HAFC-actuated bimorphic structure was created. HAFC actuators were selected in part due to their increased reliability during fibre cracking as compared with MFC actuators. An examination of the reliability of HAFCs was also undertaken.

\subsection{Macro Fibre Composite-Actuated Bimorphic Structure}

A Macro Fibre Composite-actuated bimorphic structure was created consisting of two Macro Fibre Composite (MFC) actuators (Figure 4-2(a)) bonded to an aluminum substrate (Figure 4-2(b)). Actuation was brought about with the application of an actuating electric potential of $1500 \mathrm{~V}$. Measurements were taken of the out-of-plane tip displacement. 
The macro fibre composite actuators consisted of lead zirconate titanate (PZT-5A) fibres in an epoxy matrix, sealed in Kapton. The macro fibre composite actuators were M-8528-P1 actuators provided by Smart-Materials, Inc.

The M-8528-P1 macro fibre composite actuator is illustrated in Figure 4-2(a).The M$8528-\mathrm{P} 1$ actuator has dimensions of $110 \mathrm{~mm} \times 45 \mathrm{~mm}$. The active region (the region containing the active fibres and the interdigitated electrodes) had dimensions of $85 \mathrm{~mm} \mathrm{x}$ $28 \mathrm{~mm}$. The active fibres had cross-section dimensions of $350 \mu \mathrm{m} \times 175 \mu \mathrm{m}$. The active fibres were spaced $235 \mu \mathrm{m}$ apart. The space between the active fibres is occupied by an epoxy matrix. This epoxy matrix was composed of epoxy DOW DER 330. The interdigitated electrodes of the MFC were spaced $500 \mu \mathrm{m}$ apart.

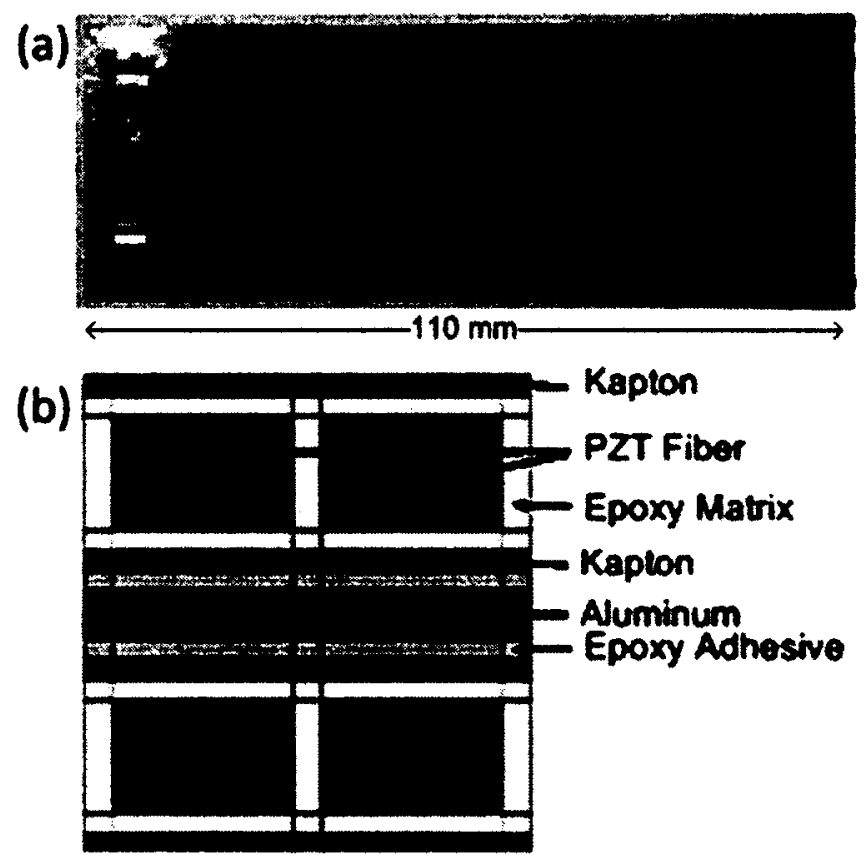

Figure 4-2: Macro fibre composite

(a) M-8528-P1 macro fibre composite actuator

(b) Cross section of bimorphic structure containing an M-8528-P1 actuator 
Thin aluminum sheet $88.9 \mu \mathrm{m}\left(0.035^{\prime \prime}\right)$ thick was used as the substrate of the bimorphic actuator.

Epoxy DOW DER 330 was used as an adhesive between the aluminum substrate and the M-8528-P1 Macro Fibre Composite Actuators. The adhesive layer had an approximate thickness of $38.1 \mu \mathrm{m}(0.0015 \mathrm{in})$.

The entire bimorphic structure had a measured thickness of $765 \mu \mathrm{m}$. The measured thicknesses of the aluminum substrate $(88.9 \mu \mathrm{m})$, the adhesive layers $(38.1 \mu \mathrm{m}$ each) and the active fibres (175 $\mu \mathrm{m}$ each) could be accounted for. The remaining $250 \mu \mathrm{m}$ was attributed to the thickness of the epoxy matrix and the surrounding Kapton layers. Hence, the epoxy matrix and Kapton layers were attributed a thickness of $31.25 \mu \mathrm{m}$ each.

The Macro Fibre Composite-actuated bimorphic structure was clamped in a fixture. An actuating electric potential difference of $1500 \mathrm{~V}$ was established between the interdigitated electrodes using a voltage amplifier (not shown). An axial load of $15 \mathrm{~N}, 30$ $\mathrm{N}$ and $40 \mathrm{~N}$ was measured and applied to the structure using a series of pre-stressed rubber bands. The tip displacement of the structure was measured using a Keyence LK086 laser displacement sensor. The Keyence LK-086 had a measurement range of $30 \mathrm{~mm}$ and a resolution of $3 \mu \mathrm{m}$. Displacement was measured at the edge of the active region of bimorphic actuator. The test setup is illustrated in Figure 4-3. The resulting tip displacements are presented in Figure 4-8. 


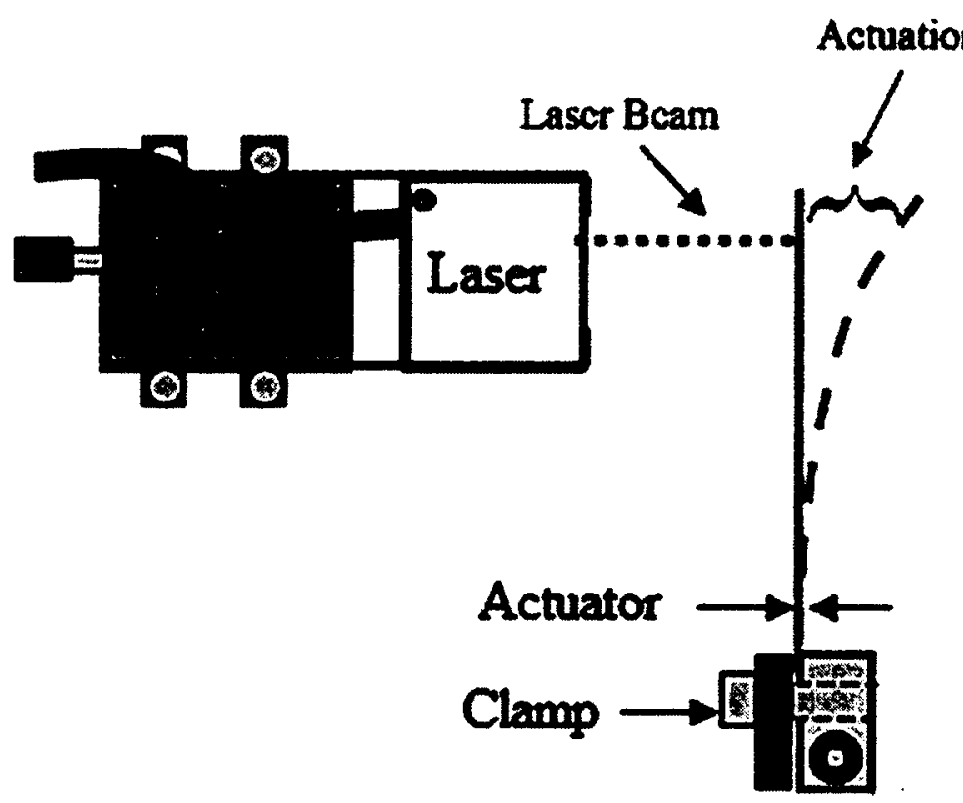

Figure 4-3: Experimental setup [54]

An analytical model predicting the tip deflections of the MFC-actuated bimorphic structure was created by Dr. Eric Chen of the Institute of Aerospace Research at the National Research Council Canada. The analytical model was created using equations set out in [52] and [53]. A composite beam containing piezoelectric materials is illustrated in Figure 4-4. 


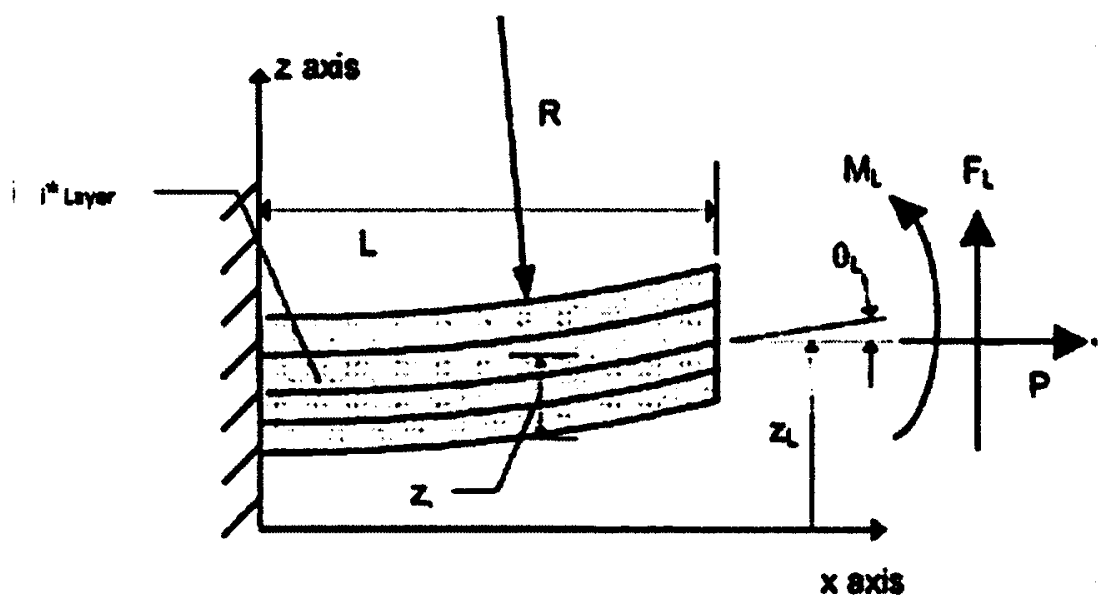

Figure 4-4: Composite beam undergoing tip deflection due to applied load and piezoelectric stresses [53]

The displacement distribution of a composite structure is given as:

$$
\frac{d^{2} z}{d x^{2}}=\frac{T_{L}(L-x)+M_{L}-\sum_{i} E_{i} Z_{i} A_{i} d_{33} V_{i}-T_{a}\left(z_{L}-z(x)\right)}{E I}
$$

Where,

- $T_{L}$ is the lateral load

- $M_{L}$ is the moment

- $E_{i}$ is the Young's modulus of layer $i$

- $A_{i}$ is the cross-sectional area of layer $i$

- $d_{33}$ is the piezoelectric coupling coefficient

- $V_{i}$ is the electric field at layer $i$

- $T_{a}$ is the axial load

- $E$ is the composite young's modulus

- $\quad$ I is the second moment of area 
The parameters used in the model are tabulated in Table 4-1. The cross-sectional area of a layer, $A_{i}$, is determined by the thickness of the layer and by the width of the beam. The bimorph was assigned a width of $35 \mathrm{~mm}$ and a length, $L$, of $95 \mathrm{~mm}$.

Table 4-1: Material parameters of the bimorphic structure

\begin{tabular}{cccc}
\hline Material & Young's Modulus, E (Pa) & Thickness, $t(\mathrm{~m})$ & Piezoelectric Coupling, d31 (V/m) \\
\hline PZT & $61.52 \times 10^{9}$ & $191 \times 10^{-6}$ & $171 \times 10^{-12}$ \\
Aluminum & $70 \times 10^{9}$ & $76 \times 10^{-6}$ & 0 \\
Adhesive & $3.378 \times 10^{9}$ & $31 \times 10^{-6}$ & 0
\end{tabular}

The mathematically-predicted tip deflections are also presented in Figure 4-8.

A finite element model of the MFC-actuated bimorphic structure was also created.

\subsection{Finite Element Model of Macro Fibre Composite-Actuated}

\section{Bimorphic Structure}

A finite element analysis of the bimorphic structure was produced. The goal of the finite element analysis was to determine the tip displacement performance of the actuated bimorphic structure. A representative finite element model was created. The finite element analysis was simplified and reduced to the width of a single active fibre (plus a proportional quantity of accompanying, surrounding, inactive materials). The reduced finite element model of the bimorphic structure was composed of 15,288 three dimensional, 20-node, piezoelectric (C3D20E) elements and had dimensions of $98 \mathrm{x}$ $0.585 \times 0.765 \mathrm{~mm}$. The electrodes were $85 \mu \mathrm{m}$ in width and were separated by a $415 \mu \mathrm{m}$ space. 
The physical bimorphic actuator was clamped at one end. The finite element model only included the segment of the actuator that was not clamped. Hence, the finite element model was $98 \mathrm{~mm}$ long while the physical actuator was $114 \mathrm{~mm}$ long.

The finite element model used a mesh of 15,288 C3D20E elements $(13 \times 3 \times 4$ elements per $\mathrm{mm}, 98 \mathrm{~mm}$ long). The representative finite element model is show in Figure 4-5.

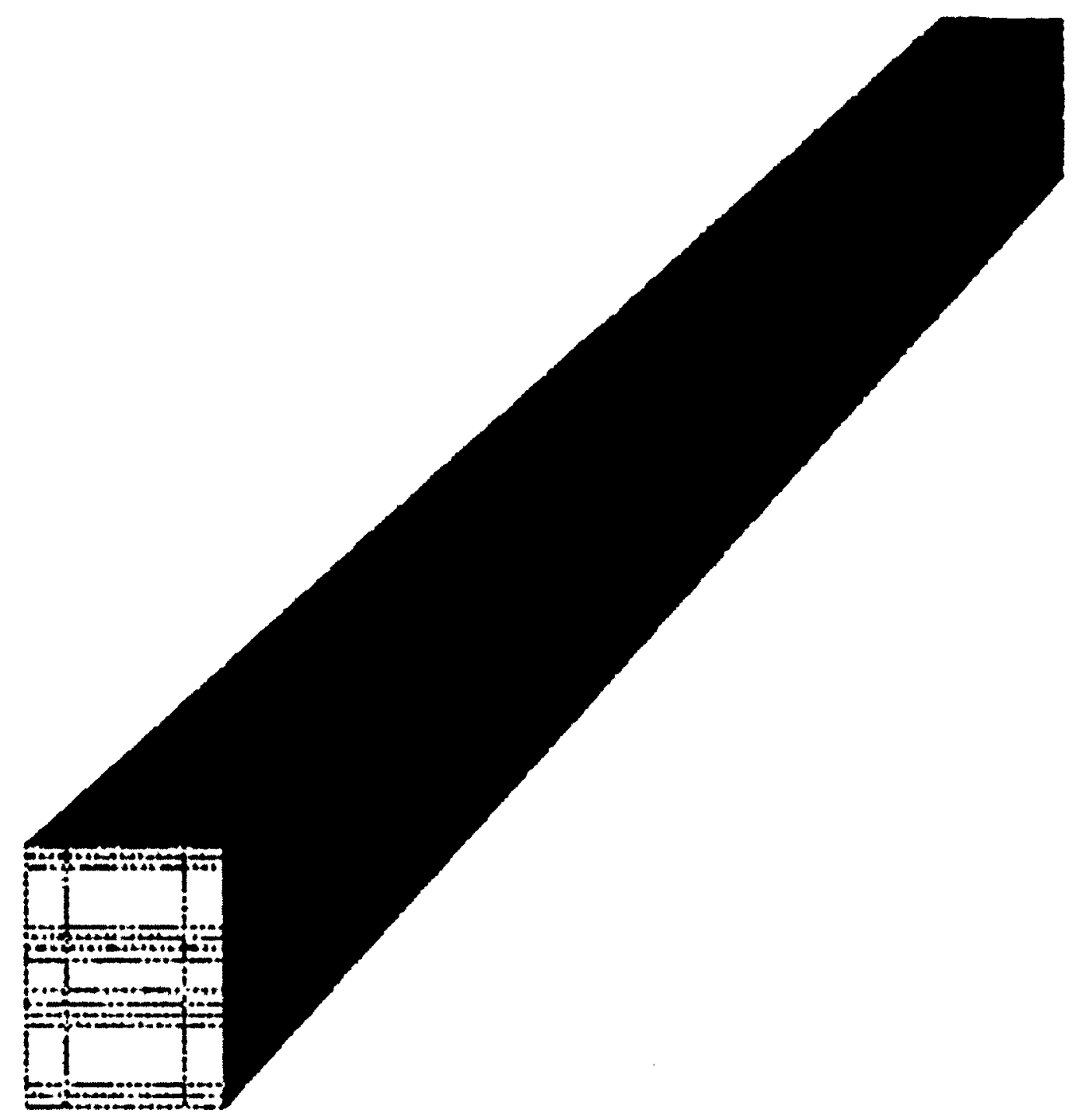

Figure 4-5: Finite element model of the MFC-actuated bimorphic structure 
A cross-section of the finite element model of the bimorphic structure, with dimensions, is illustrated in Figure 4-6.

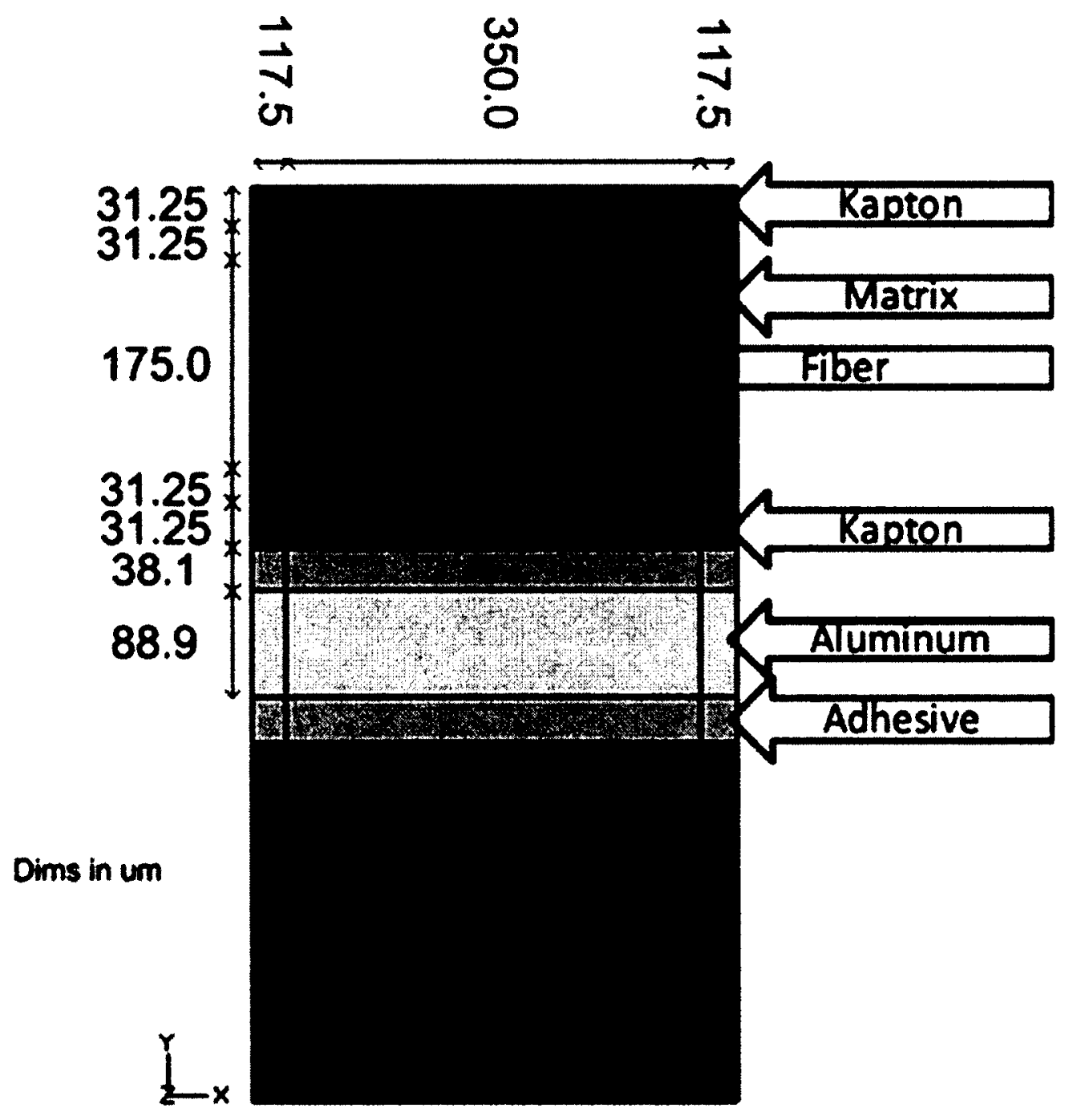

Figure 4-6: Cross-section of the finite element model

In order to remain consistent with the physical bimorph, $13 \mathrm{~mm}$ of the model, starting from the unclamped end, was made inactive. In the inactive length of the model, the piezoelectric fibre material was removed, replaced with epoxy matrix material. 
The material properties used in the finite element model of the bimorphic structure are listed in Table 4-2.

Table 4-2: Material properties of active fibre composite bimorph structure

\begin{tabular}{crrrrrr}
\hline & Aluminum & \multicolumn{1}{c}{ Adhesive } & Kapton & Matrix Epoxy & Piezoelectric Fibre \\
\hline & & DOW DER 330 & DuPont 100-HN & DOW DER 330 & PZT-5A \\
\hline Young's modulus (GPa) & 70.00 & 3.38 & 2.05 & 3.38 & 60.90 \\
\hline Poisson's ratio & 0.33 & 0.33 & 0.36 & 0.33 & 0.35 \\
\hline Permittivity & & & & & \\
K11 (F/m) & $6.20 E-05$ & $3.52 \mathrm{E}-06$ & $3.01 \mathrm{E}-11$ & $3.52 \mathrm{E}-05$ & $1.53 \mathrm{E}-08$ \\
K22 (F/m) & $6.20 \mathrm{E}-05$ & $3.52 \mathrm{E}-06$ & $3.01 \mathrm{E}-11$ & $3.52 \mathrm{E}-05$ & $1.53 \mathrm{E}-08$ \\
K33 (F/m) & $6.20 \mathrm{E}-05$ & $3.52 \mathrm{E}-06$ & $3.01 \mathrm{E}-11$ & $3.52 \mathrm{E}-05$ & $1.51 \mathrm{E}-08$ \\
\hline Piezoelectric Coefficients & & & & & \\
d15, d24 (pC/N) & 0.00 & 0.00 & 0.00 & 0.00 & 585.00 \\
d31, d32 (pC/N) & 0.00 & 0.00 & 0.00 & 0.00 & -171.00 \\
d33 (pC/N) & 0.00 & 0.00 & 0.00 & 0.00 & 374.00
\end{tabular}

The actuation of the Active Fibre Composite bimorph structure was achieved by applying an electric potential of $1500 \mathrm{~V}$ to alternating electrodes. Other electrodes were grounded. On one side of the aluminum substrate, the piezoelectric fibre was poled in the same direction as the applied electric field, resulting in fibre extension. On the other side of the aluminum substrate, the piezoelectric fibre was poled in the direction opposite to that of the applied electric field, resulting in fibre contraction. This coupling of fibre extension and contraction produced the out-of-plane bending of the bimorphic structure. The actuation of the bimorphic structure was further amplified by the application of an axial compressive load to the structure. These load conditions are illustrated in Figure 47. 


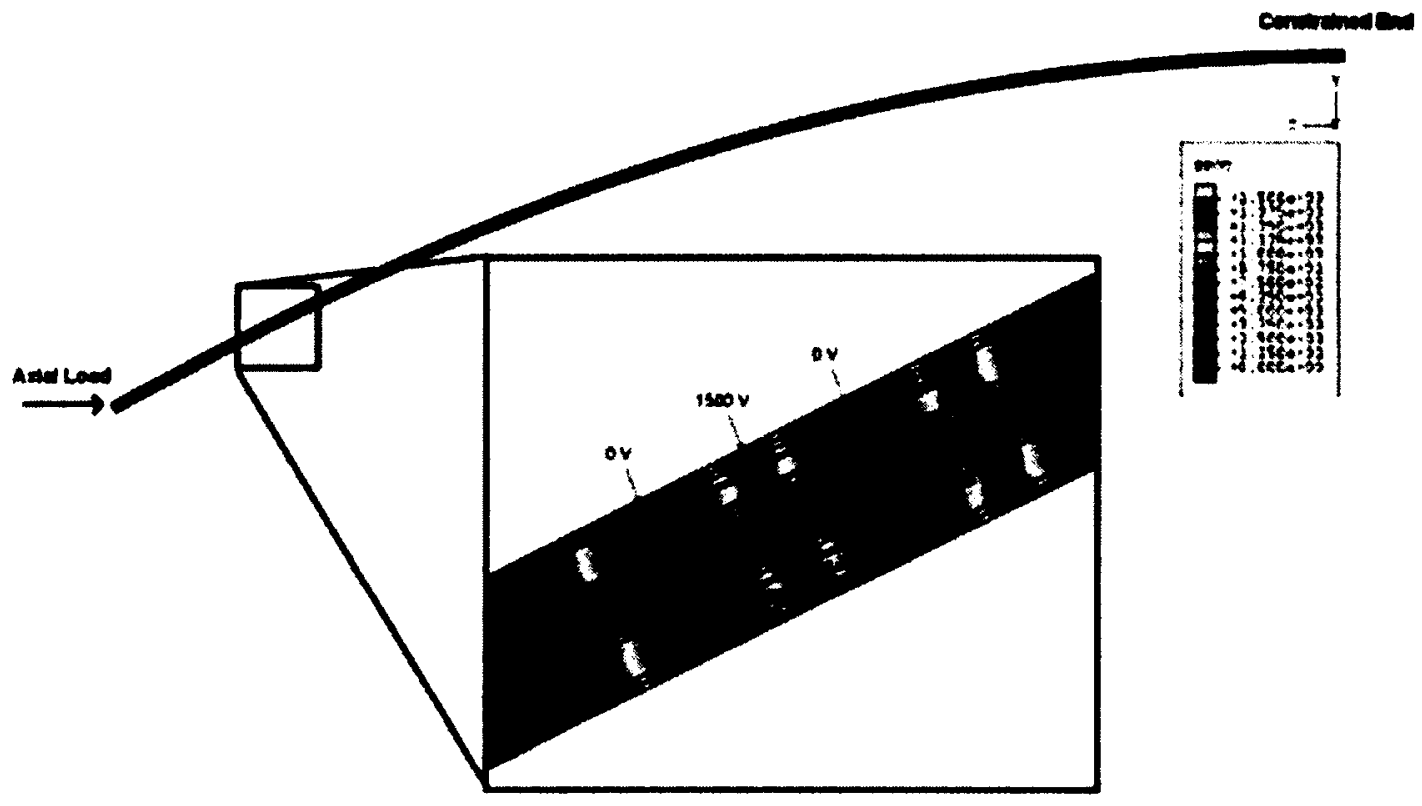

Figure 4-7: Load conditions applied to active fibre bimorph structure

The finite element analysis was conducted using the manufacturer-suggested actuation potential of $1500 \mathrm{~V}$. The theoretical maximum actuating potential that could be applied to the material is $2000 \mathrm{~V}$. The finite element analysis was also conducted using this theoretical maximum actuation potential of $2000 \mathrm{~V}$.

The resulting deflections of the finite element model bimorph are compared to the results for the physical bimorph deflection in Figure 4-8. There is a strong agreement between results obtained by the physical model, the finite element model and the analytical model. This strong agreement indicates that the tip deflection resulting from an actuation potential of $1500 \mathrm{~V}$ is correct. Given that the finite element model produced correct results for an actuating potential of $1500 \mathrm{~V}$, it can be assumed that the finite element model will also produce correct results for an actuating potential of $2000 \mathrm{~V}$, assuming no hysteresis. 


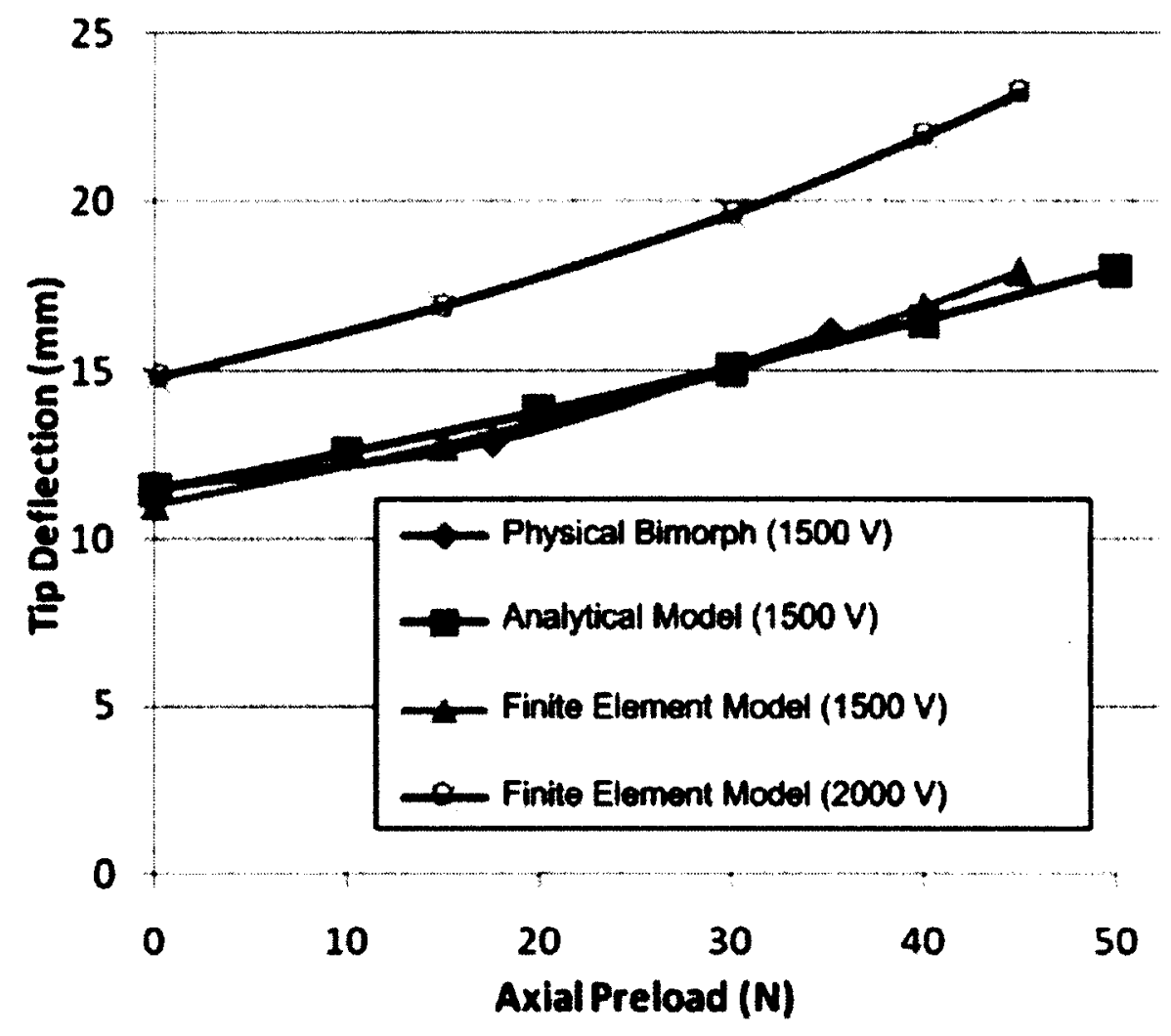

Figure 4-8: Tip deflection of an MFC-actuated bimorph subjected to an axial preload and an actuating potential of either $1500 \mathrm{~V}$ or $2000 \mathrm{~V}$

As shown in Figure 4-8, the MFC-actuated bimorph achieved an out-of-plane tip displacement of $11.1 \mathrm{~mm}$ due to the manufacturer-suggested actuation potential of 1500 $\mathrm{V}$ and without axial preload. This out-of-plane tip displacement could be amplified to 16 $\mathrm{mm}$ using an axial preload of $40 \mathrm{~N}$.

The maximum actuating electric potential theoretically sustainable by the PZT material is $2000 \mathrm{~V}$. The MFC-actuated bimorph achieved an out-of-plane tip displacement of $14.77 \mathrm{~mm}$ due to this actuation potential of $2000 \mathrm{~V}$ and without axial 
preload. This out-of-plane tip displacement could be amplified to $23.2 \mathrm{~mm}$ using an axial preload of $40 \mathrm{~N}$.

\subsection{Finite Element Model of Hollow Active Fibre Composite (HAFC)} Actuator

A finite element analysis was conducted to determine the out-of-plane bending performance of a Hollow Active Fibre Composite (HAFC)-actuated bimorphic structure.

HAFC actuators were selected in part due to their increased reliability during fibre cracking as compared with MFC actuators [8]. The reliability of HAFC during fibre cracking was examined and presented in [8].

\subsubsection{HAFC Actuator Reliability During Fibre Cracking}

A finite element model was created to study the effect of transverse fibre cracking on HAFC. Transverse fibre cracking is a common problem challenging the reliability of MFCs. MFCs tend to develop transverse fibre cracks near the interdigitated electrodes due to inhomogeneous electric fields during poling. Transverse fibre cracking is not the only composite failure mechanism that can occur in MFC, however, it is the most common. Other mechanism of composite failure in MFC include fiber delamination

The model consisted of a lamina of hollow piezoelectric fibres embedded in an epoxy matrix. The lamina is illustrated in Figure 4-9. The lamina was assigned dimensions of 77 $\mathrm{mm}$ long $2.8 \mathrm{~mm}$ tall $16.85 \mathrm{~mm}$ wide. The hollow fibres embedded in the lamina had a diameter of $900 \mu \mathrm{m}$ and wall thickness of $190 \mu \mathrm{m}$. The fibres were assigned the material properties of PZT-5H and had a Young's Modulus of $E_{11}=E_{22}=60.6 \times 10^{9} \mathrm{~Pa}$ and $\mathrm{E}_{33}=$ 
$48.3 \times 10^{9} \mathrm{~Pa}$, Poisson's ratios of $\mu_{12}=0.2896$ and $\mu_{13}=\mu_{23}=0.408$, shear moduli of $\mathrm{G}_{12}=23.6 \times 10^{9} \mathrm{PA}$ and $\mathrm{G}_{13}=\mathrm{G}_{23}=2.29 \times 10^{9} \mathrm{~Pa}$, permittivities of $\mathrm{k}_{11}=\mathrm{k}_{22}=2.77 \times 10^{-}$ ${ }^{8} \mathrm{~F} / \mathrm{m}$ and $\mathrm{k}_{33}=3.01 \times 10^{-8} \mathrm{~F} / \mathrm{m}$ and piezoelectric coefficients of $d_{15}=d_{24}=741 \times 10^{-12}$ $C / N, d_{31}=d_{32}=-274 \times 10^{-12} \mathrm{C} / \mathrm{N}$ and $d_{33}=593 \times 10^{-12} \mathrm{C} / \mathrm{N}$. Polarization direction about the fibre circumference was taken into account when assigning these material properties. The matrix material was assigned the material properties of Insulcast $501 /$ Insulcure 24 and had a young's modulus of $E=1.29 \times 10^{9} \mathrm{~Pa}$ and a Poisson's ratio of $\mu=0.3$.

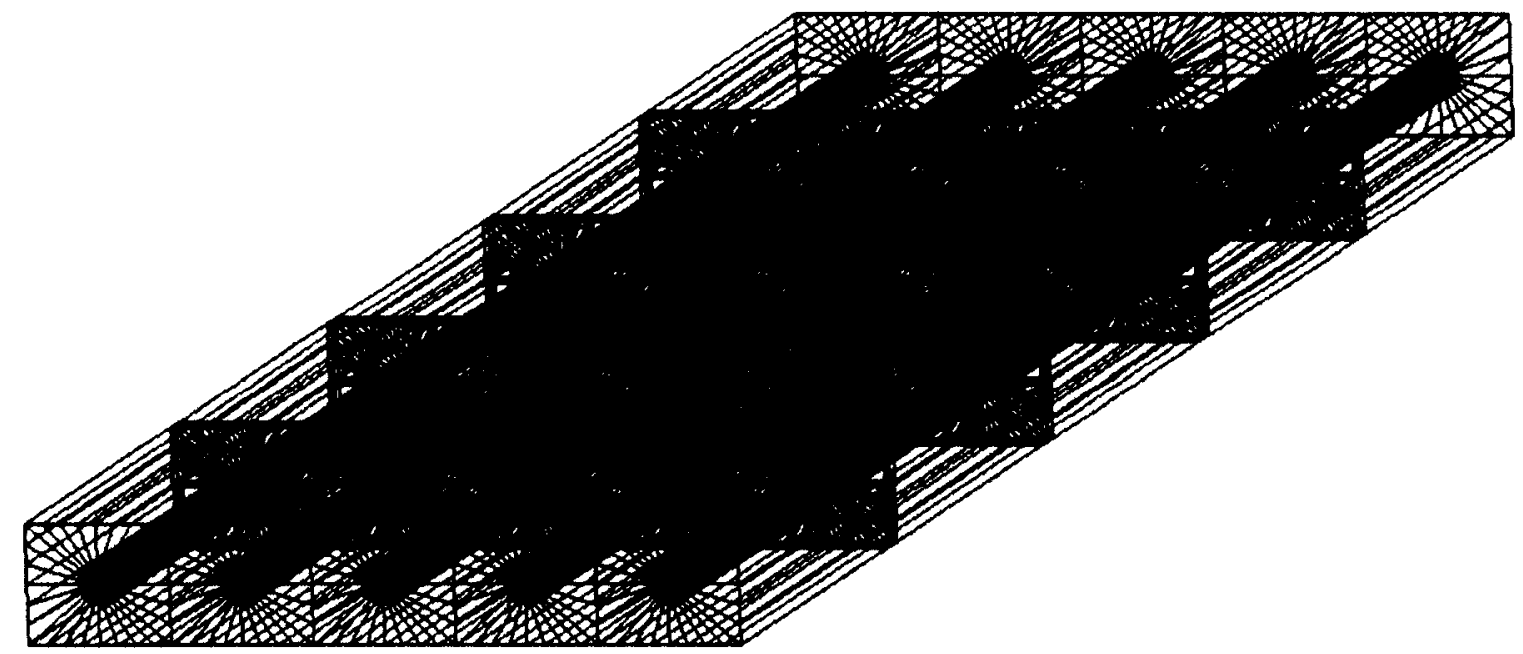

Figure 4-9: Finite element model of HAFC lamina [8]

An electrode bus, located at both ends of the lamina could establish potential difference of $57 \mathrm{~V}(300 \mathrm{~V} / \mathrm{mm})$ between the inner and outer walls of the hollow active fibres. The fibres were polled in the radial direction. This electric potential difference and poling are illustrated in Figure 4-10. The actuation potential caused an extension of the lamina. 


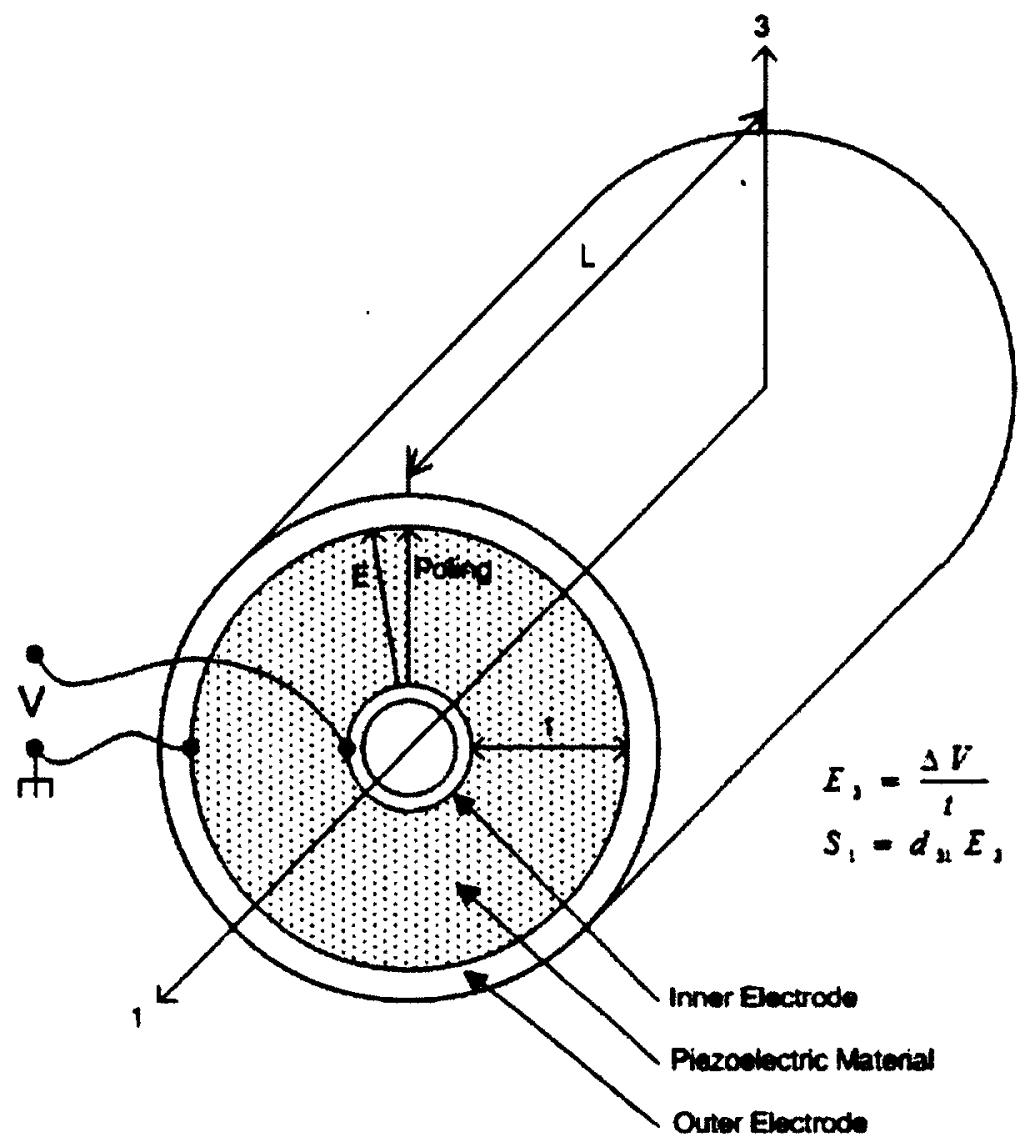

Figure 4-10: Hollow active fibre with electrode placement and poling

Cracks were simulated in the fibres of the lamina. The cracks regions were modeled so that the piezoelectric material was removed and substituted for an epoxy material with $1 / 1000^{\text {th }}$ of the stiffness of the matrix epoxy material. The crack material had a Young's Modulus of $\mathrm{E}=1.29 \times 10^{9} \mathrm{~Pa}$ and a Poisson's ratio of $\mu=0.3$. The crack isolated a segment of the piezoelectric material from the actuating electric potential. The electric potential placement and the effect of the crack is illustrated in Figure 4-11. The degradation of the elongation performance of the lamina was studied as cracks were introduced. The results are presented in Figure 4-12. 

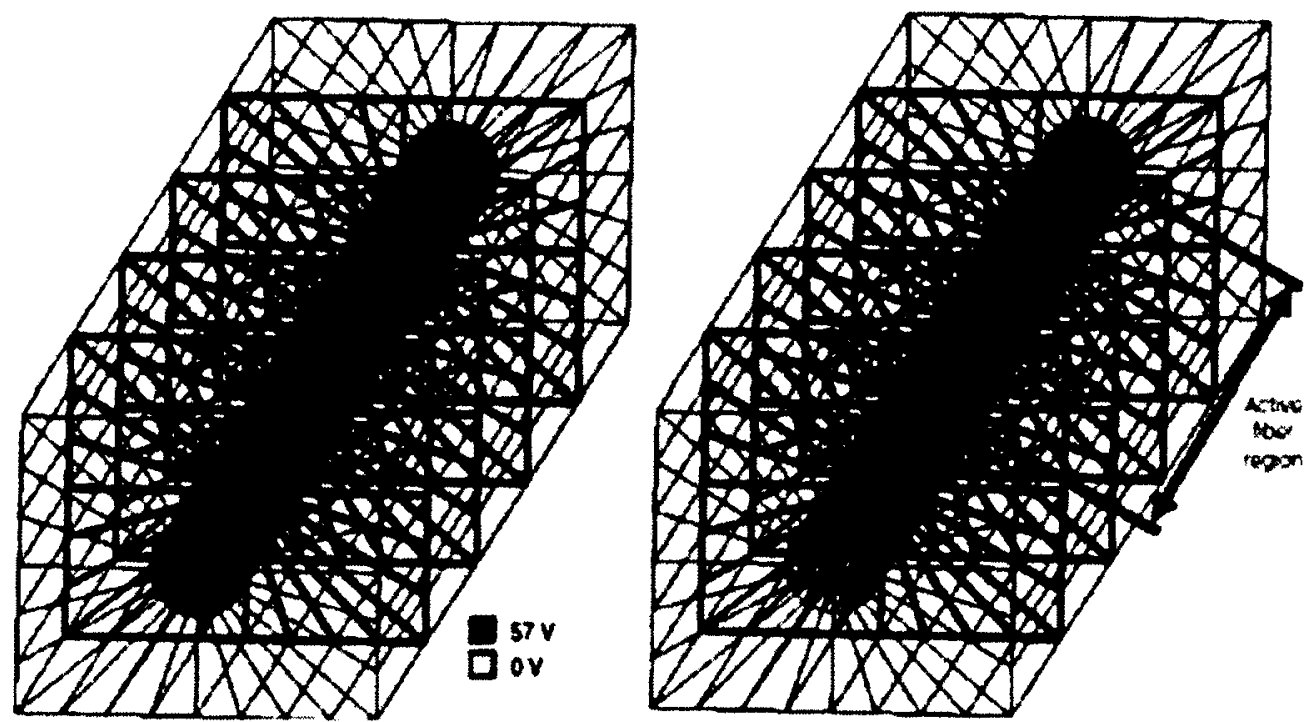

Figure 4-11: Broken fibre effects [8]

(a) HAFC lamina with electric potential and

(b) Cracked HAFC with electric potential removed from inactive regions

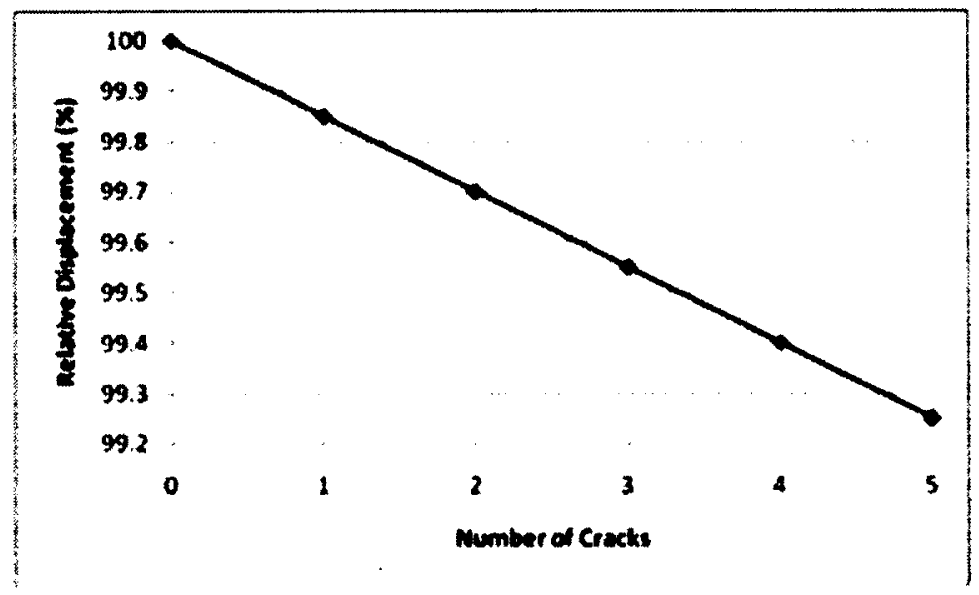

Figure 4-12: Lamina extension performance with introduction of fibre cracks [8]

The lamina underwent $100.0 \%$ extension when no fibre cracks were present. When five cracks were present, the lamina extension performance degraded to $99.3 \%$ of that of the undamaged lamina. The lamina undergoes little elongation performance degradation. 
The lamina suffers very little extension performance degradation as cracks are introduced. With the dual electrode bus system, the active fibres are not separated from the actuating electric potential and therefore do not suffer much loss of actuation performance.

The reliability and performance of HAFC lamina during sensing was also examined.

\subsubsection{HAFC Sensor Reliability During Fibre Cracking}

The finite element of the model used in section 4.3.1 was reused in order to examine the reliability of HAFC lamina during sensing. The lamina was fully constrained at one end, while the other end was subjected to a fixed extension of $35 \mathrm{~mm}$ ( 45.4 microstrain). The potential difference established between the inner and outer walls of the fibre was monitored as cracks were introduced to the fibres. The results are illustrated in Figure 413. The lamina, undergoing sensing is illustrated in Figure 4-14.

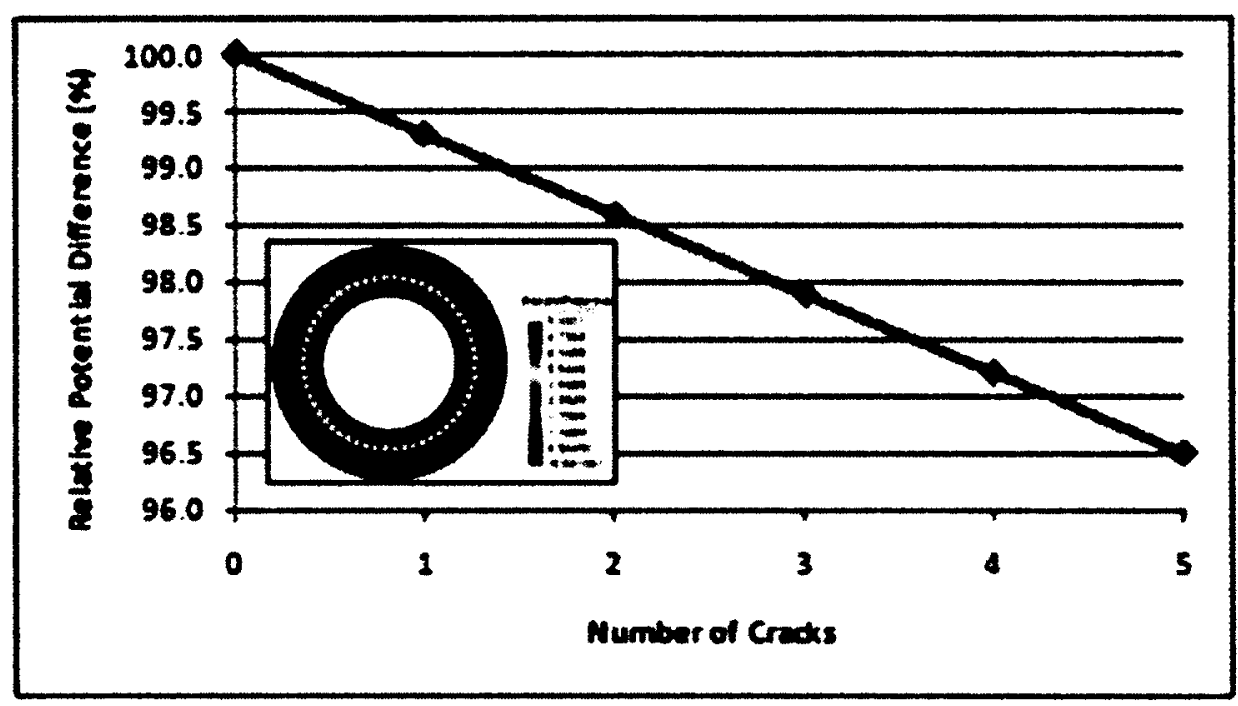

Figure 4-13: Lamina sensing performance with introduction of fibre cracks [8] 
The lamina underwent $100.0 \%$ extension when no fibre cracks were present. When five cracks were present, the lamina extension performance degraded to $96.5 \%$ of that of the undamaged lamina. The lamina undergoes little sensing performance degradation as cracks are introduced.

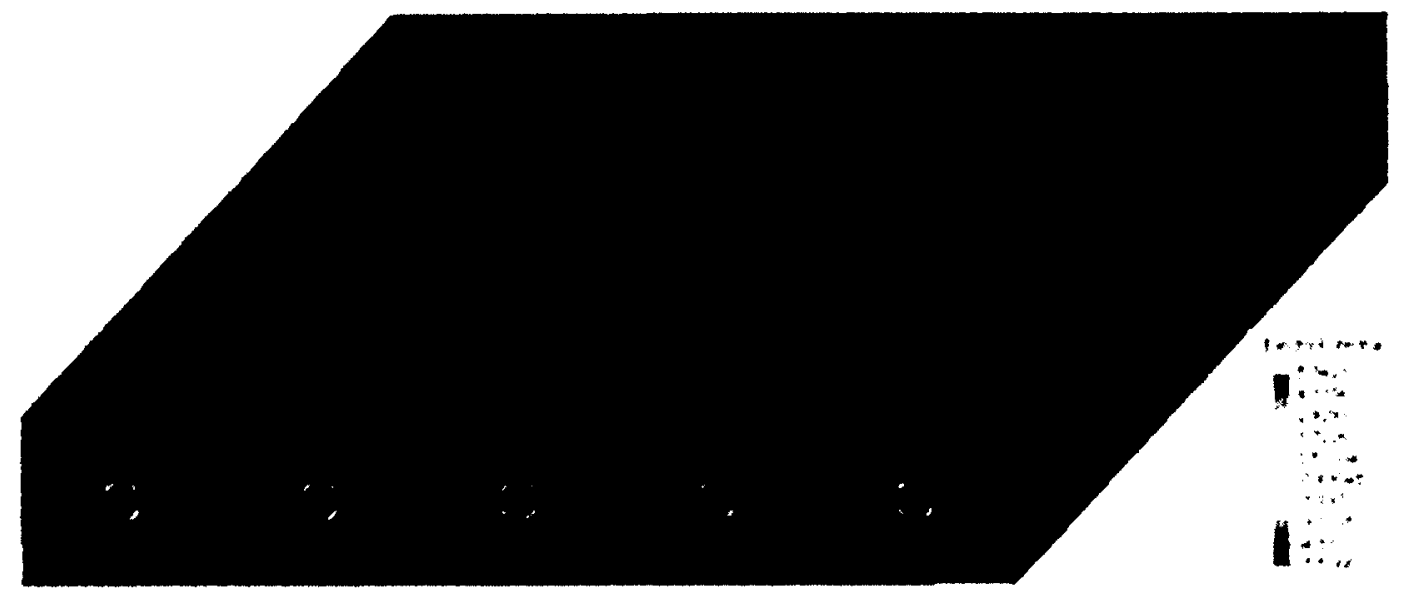

Figure 4-14: Voltage distribution on damaged sensor lamina [8]

HAFC actuators have been proven to be very reliable during actuation. The use of HAFCs to actuate a bimorphic structure has been explored.

The examination of the reliability of HAFC jpresented here was also presented in [8].

\subsubsection{Finite Element Analysis of Hollow Active Fibre Composite-Actuated Bimorphic Structures}

The HAFC-actuated bimorph was tailored in order to be similar to the Macro Fibre Composite (MFC) -actuated bimorph analyzed in the in section 4.1. The HAFC-actuated bimorph was similar to the MFC-actuated bimorph such that the HAFC-actuated bimorph was designed to have the same fibre volume fraction as the MFC-actuated bimorph. Also, 
the actuation potential that was applied to the HAFC-actuated bimorph was selected in order to yield electric field intensity similar to that used in the MFC-actuated bimorph. The cross-section of the HAFC-actuated bimorphic structure, with dimensions, is illustrated in Figure 4-15. The dimensions of the MFC-actuated and HAFC-actuated bimorphic structures are tabulated in Table 4-2.

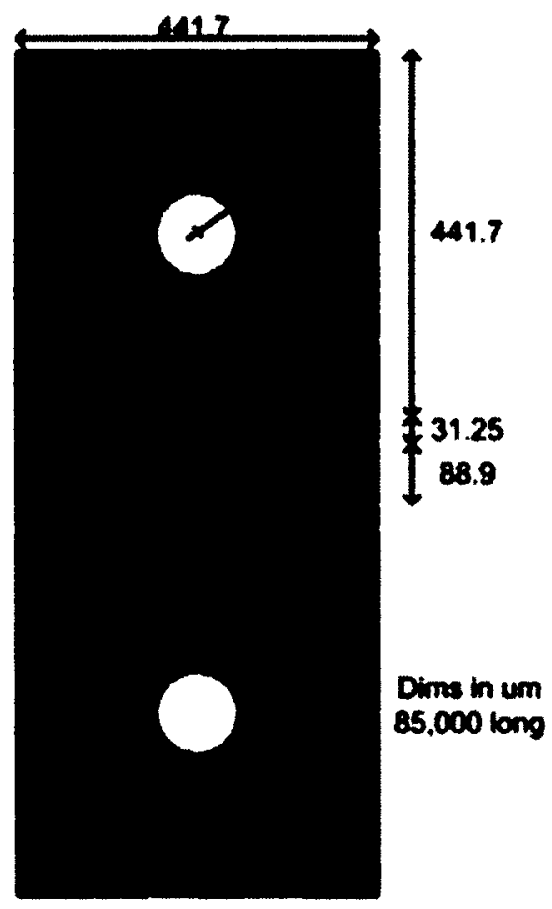

Figure 4-15: Cross-section of the HAFC-actuated bimorphic structure 
Table 4-3: Characteristics and dimensions of the MFC-and HAFC-actuated bimorphs

\begin{tabular}{lrrr} 
& $\begin{array}{l}\text { Macro fibre } \\
\text { composite-actuated } \\
\text { bimorph }\end{array}$ & $\begin{array}{l}\text { Hollow active fibre } \\
\text { composite-actuated } \\
\text { bimorph }\end{array}$ \\
\hline Aluminum thickness $(\mathrm{mm})$ & 88.9 & 88.9 \\
Adhesive thickness $(\mathrm{mm})$ & 31.25 & 31.25 \\
Fibre length $(\mathrm{mm})$ & 85 & 85 \\
Fibre height $(\mu \mathrm{m})$ & 175 & $\mathrm{n} / \mathrm{a}$ \\
Fibre width $(\mu \mathrm{m})$ & 350 & $\mathrm{n} / \mathrm{a}$ \\
Fibre inner radius $(\mu \mathrm{m})$ & $\mathrm{n} / \mathrm{a}$ & 50 \\
Fibre outer radius $(\mu \mathrm{m})$ & $\mathrm{n} / \mathrm{a}$ & 200 \\
Matrix width $(\mu \mathrm{m})$ & 585 & 441.7 \\
Matrix height $(\mu \mathrm{m})$ & 237.5 & 441.7 \\
Fibre volume fraction & 0.629 & 0.629 \\
Automatic electric potential $(\mathrm{V})$ & 1500 & 723 \\
Electric field intensity $(\mathrm{V} / \mathrm{mm})$ & 4820 & 4820
\end{tabular}

The HAFC-actuated bimorph was fully constrained at one end. The actuating electric potential difference of $723 \mathrm{~V}$ was established between the inner and outer walls of the hollow fibre. The electric potential distribution is illustrated in Figure 4-16. 


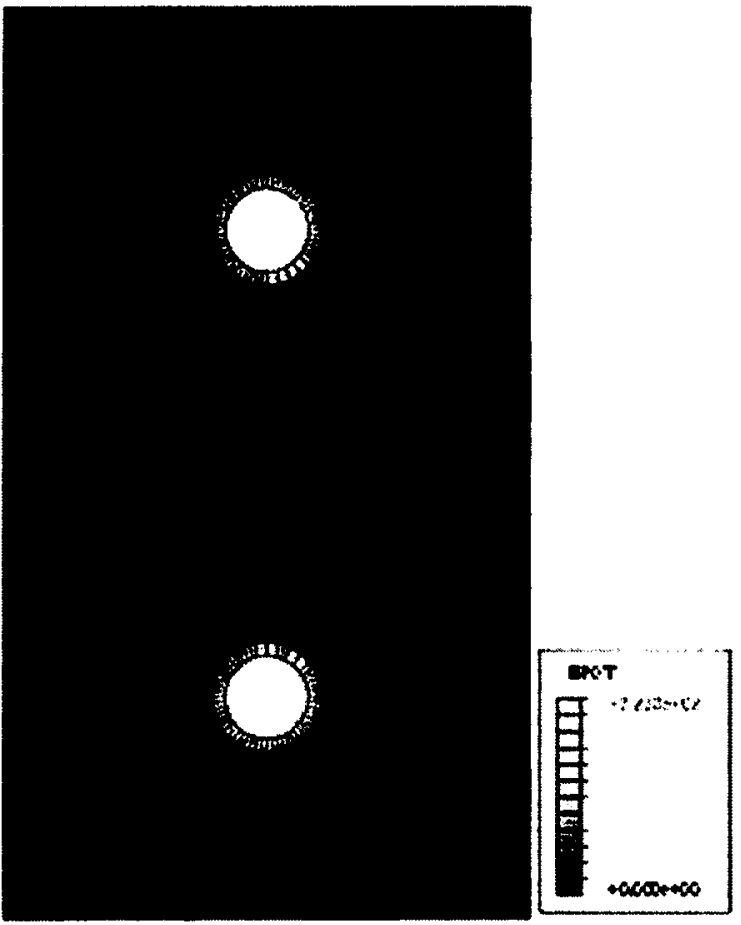

Figure 4-16: Electric potential distribution of HAFC-actuated bimorph

One of the fibres was poled such that the poling direction was parallel to the direction of the actuating electric potential. The result was the axial extension of the hollow fibre. The fibre on the opposite face of the bimorph was poled in the direction opposite to that of the actuating electric field. The result was the axial contraction of the hollow fibre. The anti-parallel actuation of the hollow fibres on the opposite faces of the bimorph resulted in the bending actuation of the bimorphic structure. The displaced structure created in the finite element analysis is illustrated in Figure 4-17. The finite element analysis produced an out-of-plane tip displacement of $7.41 \mathrm{~mm}$. 


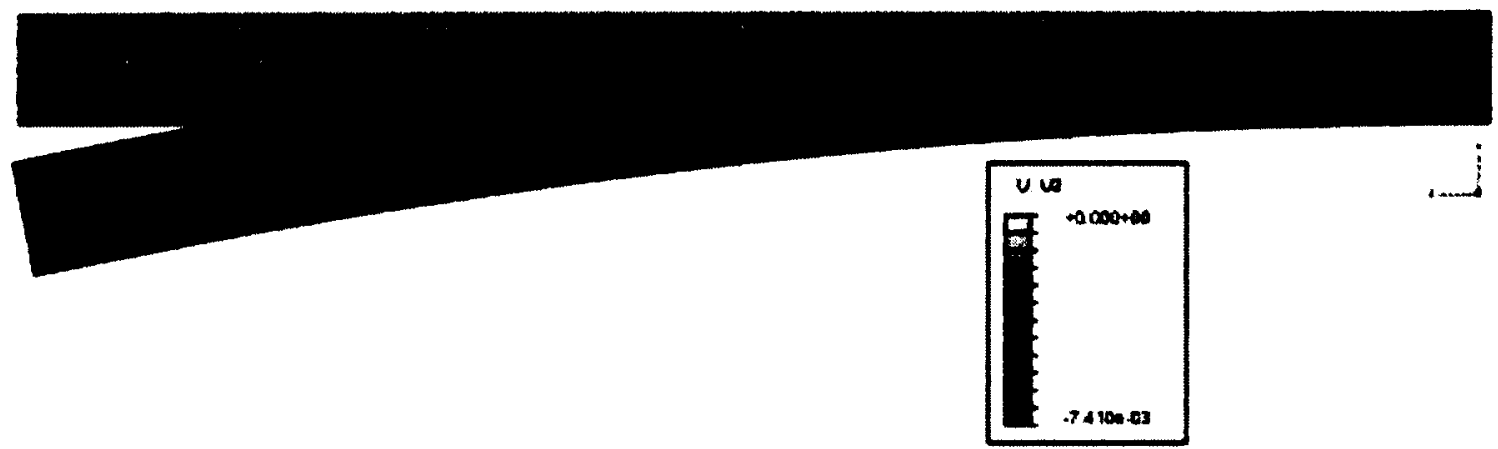

Figure 4-17: Out-of-plane tip deflection of HAFC-actuated bimorph

\subsection{Summary}

Finite element analyses were created of a Macro Fibre Composite (MFC)-actuated bimorphic structure as well as of a similar Hollow Active Fibre Composite (HAFC)actuated bimorphic structure. The MFC-actuated structure generated an out-of-plane tip displacement of $18 \mathrm{~mm}$ when exposed to an actuating electric field intensity of 4820 $\mathrm{V} / \mathrm{mm}$. An HAFC-actuated bimorphic structure was designed to have similar properties and a similar fibre volume fraction. This similar HAFC-actuated bimorphic structure generated an out-of-plane tip displacement of $7.41 \mathrm{~mm}$ when exposed to the same actuating electric field intensity of $4820 \mathrm{~V} / \mathrm{mm}$.

The bending performance of the HAFC-actuated bimorph was noticeably smaller than that of the similar MFC-actuated bimorph. This result is likely due to the piezoelectric characteristics of the fibres used in the MFCs and HAFCs. MFC actuation is proportional to the parallel piezoelectric coefficient, $d_{33}$. HAFC actuation is proportional to the orthogonal piezoelectric coefficient, $d_{31}$. Since the $d_{31}$ for the selected material was nearly half of that of the $d_{33}$ (see table 4-2), a reduction in actuation performance was expected. 
However, HAFC-based actuators offer some advantages over MFC-based actuators such as increased reliability and decreased actuation electric potential. 


\section{Chapter 5: Conclusions}

The deflection performance of a Macro Fibre Composite (MFC)-actuated and Hollow Active Fibre Composite (HAFC)-actuated bimorphic actuators were studied. The study also measured the amplification effects of axial preload on deflection performance. In order to conduct this study, a physical MFC-actuated structure was manufactured. A Finite Element Model was also created and compared to an analytical model.

The physical model consisted of two M-8528-P1 MFCs bonded to both sides of an aluminum substrate. The $98 \mathrm{~mm}$ long bimorphic structure achieved approximately $11 \mathrm{~mm}$ tip deflection $\left(6.4^{\circ}\right)$ when an actuating electric potential of $1500 \mathrm{~V}(4820 \mathrm{~V} / \mathrm{mm}$ actuating electric field intensity) was applied to the MFCs. The tip deflection of the above bimorphic structure would increase to approximately $17 \mathrm{~mm}\left(9.8^{\circ}\right)$ when an axial preload of $40 \mathrm{~N}$ was applied in addition to the $1500 \mathrm{~V}$ actuating electric potential. The finite element and analytical models produced similar results. The finite element model further predicted that a tip deflection of $23 \mathrm{~mm}\left(13.2^{\circ}\right)$ could be achieved with the application of an axial compressive force of $80 \mathrm{~N}$ and an actuating electric potential of $1500 \mathrm{~V}$. The finite element model also predicted that a tip deflection of $15 \mathrm{~mm}\left(13.2^{\circ}\right)$ could be achieved with the application of an actuating electric potential of $2000 \mathrm{~V}$. 
The deflection performance of a Hollow Active Fibre Composite (HAFC) -actuated bimorphic structure was also studied. A finite element model of an HAFC was created and was assigned material properties, fibre volume fraction and actuating electric field intensity similar to that of the M-8528-P1 MFC-actuated bimorphic structure. The HAFC-actuated bimorphic structure was exposed to a $4820 \mathrm{~V} / \mathrm{mm}$ actuating electric field intensity. The $98 \mathrm{~mm}$ bimorph achieved a tip deflection of $7.41 \mathrm{~mm}\left(4.32^{\circ}\right)$. The tip deflection performance of the HAFC-actuated bimorphic structure was approximately half of that of the MFC-actuated bimorphic structure. The finite element model confirmed this result.

The actuation performance of an HAFC is proportional to the orthogonal piezoelectric coefficient $d_{31}$. Meanwhile, the actuation performance of an MFC is proportional to the parallel piezoelectric coefficient $d_{33}$. Since the value of $d_{31}$ of the PZT studied was approximately half of the value of the $d_{33}$, a decrease in tip deflection performance was anticipated.

HAFCs provide many advantages over MFCs, such as increased reliability, resistance to fibre breakage and lower required actuation electric potential. In some cases, these advantages of HAFCs could outweigh any decreases in actuation performance when compared with MFCs.

It has been shown that MFC and HAFC-based bimorphic structures deliver large actuation performance. It has also been shown that the actuation performance of the bimorphic structures could be amplified with the use of a compressive axial preload. The 
actuation performance of these bimorphic structures suggest that they could be potential candidates to be morphing wing actuators.

The results were published in:

Martinez, M., Kernaghan, R. Artemev, A., "Finite Element Analysis of Broken Fibre Effects on Hollow Active Fibre Composites, Journal of Intelligent Material Systems and Structures, Vol. 21, pp. 107-113, 2010.

Wickramasinghe, V.K., Chen, Y., Martinez, M., Wong, F., Kernaghan, R., "Design and Verification of a Smart Wing for an Extremely Agile Micro-Air-Vehicle", Accepted for publication to Journal of Smart Material Structures, Aug. 2011.

Wickramasinghe, V.K., Chen, Y., Martinez, M., Kernaghan, R., Wong, F., "Design and Verification of a Smart Wing for an Extremely Agile Micro-Air-Vehicle", Proceedings of: $50^{\text {th }}$ AIAA/ASME/ASCE/AHS/ASC Structures, structural Materials and Dynamics Conference, art. No. 2009-2132, 2009.

Wickramasinghe, V.K., Chen, Y., Martinez, M., Kernaghan, R., Wong, F., "Design and Verification of a Smart Wing for an Extremely Agile Micro-Air-Vehicle", ICAST 2009. 


\section{Chapter 6: Réferences}

[1] C. Niezrecki, et al., "Piezoelectric Actuation: State of the Art", Shock and Vibration Digest, July 2001, pgs 269-280.

[2] V. K. Wickramasinghe, et al., "Design and Verification of a Smart Wing for an Extremely-Agile Micro-Air-Vehicle", Conference Paper, AIAA/ASME/ASCE/AHS/ASC Structures, Structural Dynamics, and Materials Conference, California, May 2009.

[3] C. L. Sun, et al., "A Novel Drum Piezoelectric-Actuator" Applied Physics A., Vol. 84, 385-389, June 2006.

[4] B. J. Cannon, D. Brei, "Feasibility Study of Microfabrication by Coextrusion (MFCX) Hollow Fibers for Active Composites" Journal of Intelligent Material Systems and Structures, Vol. 11, 2000.

[5] F. G. Yuan, "Enhancement of Buckling Load with the Use of Active Materials", Department of Mechanical and Aerospace Engineering, North Carolina State University, Raleigh, North Carolina, December 2002.

[6] J. Sirohi, I. Chopra, "Design and Development of a High Pumping Frequency Piezoelectric-Hydraulic Hybrid Actuator", Journal of Intelligent Materials and Systems, Vol. 14, 2003. 
[7] Q. Cui, C Liu, X. F. Xha, "Modeling and Numerical Analysis of a Circular Piezoelectric Actuator for Valveless Micropumps", Journal of Intelligent Materials and Systems, Vol. 19, 2008.

[8] M. Martinez, et al., Finite Element Analysis of Broken Fibre Effects on Hollow Active Fibre Composites", Journal of Intelligent Material Systems and Structures, Vol. $21,2010$.

[9] M. Egashira, N. Shinya, "Local Strain Sensing Using Piezoelectric Polymer", Journal of Intelligent Materials and Systems and Structures, Vol. 4, 1993.

[10] M. Kobayashi, et al., "Structural Health Monitoring of Composites Using Integrated and Flexible Piezoelectric Ultrasonic Transducers", Journal of Intelligent Materials and Systems and Structures, Vol. 20, 2009.

[11] A. J. Brunner, et al., "Piezoelectric Fibre Composites as Sensor Elements for Structural Health Monitoring and Adaptive Material Systems", Journal of Intelligent Materials and Systems and Structures, Vol. 20, 2009.

[12] Y. Zhang, "In Situ Fatigue Crack Detection using Piezoelectric Paint Sensor", Journal of Intelligent Materials and Systems and Structures, Vol. 17, 2006.

[13] W. W. Clark, "Vibration Control with State-Switched Piezoelectric Materials", Journal of Intelligent Materials and Systems and Structures, Vol. 11, 2000.

[14] V. Giurgiutiu, "Review of Smart-Materials Actuation Solutions for Aeroelastic and Vibration Control", Journal of Intelligent Materials and Systems and Structures, Vol. 11, 2000. 
[15] J. Qui, M. Haraguchi, "Vibration Control of a Plate using a Self-sensing

Piezoelectric Actuator and an Adaptive Control Approach" Journal of Intelligent Materials and Systems and Structures, Vol. 17, 2006.

[16] A. Badel, et al., "Self-sensing High Speed Controller for Piezoelectric Actuator", Journal of Intelligent Materials and Systems and Structures, Vol. 19, 2008.

[17] J. Qui, et al., "Suppression of Noise Radiation from a Plate Using Self-Sensing Actuators", Journal of Intelligent Materials and Systems and Structures, Vol. 16, 2005.

[18] H. J. Kim, et. al. "High Performance Piezoelectric Microspeakers and Thin Speaker Array System" ETRI Journal, Vol. 31, December 2009.

[19] G. Furguson, et. al., "Acoustic Sensing of Direct and Indirect Weapon Fire", International Conference Series on Intelligent Sensors, Sensor Networks and Information Processing, Melbourne, 2007.

[20] Z. L. Wang, J. Song, "Piezoelectric Nanogenerators Based on Zinc Oxide Nanowire Arrays", Science 312, 242, 2006.

[21] H. A. Sodano, "Harvesting Energy from the Straps of a Backpack Using Piezoelectric Materials", Energy Harvesting Technologies, pgs. 431 -458, 2009.

[22] N. E. Dubois, Performance OF Microfabricated Piezoelectric Vibration Energy Harvesters", Integrated Ferroelectrics, Vol 83, 2006.

[23] S. R. Anton, H. A. Sodano, "A Review of Power Harvesting Using Piezoelectric Materials (2003 - 2006),"' Smart Materials and Structures, Vol. 16, 2007.

[24] X. Yuhuan, "Ferroelectric Materials and Their Applications", Elesevier Science Publishers, New York, 1991. 
[25] Unknown Author, "PI Datasheet: Fundamentals of Piezoelectricity", [Online document, available:

http://www.physikinstrumente.com/en/products/prdetail.php?sortnr=400600.00, accessed: August 2009]

[26] V. Piefort, "Finite Element Modelling of Piezoelectric Active Structures", PhD Thesis, Department of Mechanical Engineering and Robotics, Université Libre des Bruxelles, 2000-2001.

[27] J.F. Nye, "Physical Properties of Crystals: Their Representations by Tensors and Matrices”, Oxford University Press, New York, 1964.

[28] Unknown author, "Constitutive Transforms" [Online document, available: http://www.efunda.com/materials/piezo/piezo_math/transforms.cfm, accessed: July 2009] [29] A. Preumont, "Vibration control and active structures", Dordrecht, The Netherlands: Kluwer Academic Publishers, 1997.

[30] A. Bent, "Active Fiber Composites for Structural Actuation", $\mathrm{PhD}$ thesis, Department of Aeronautics and Astronautics, Massachusetts Institute of Technology, 1997.

[31] Unknown author, "Nondestructive evaluation lab", [Online document, available: http://www.me.nctu.edu.tw/ndelab/research-b.html, accessed: August 2009]

[32] X. Kornmann, et al., "Piezoelectric ceramic fibers for active fiber composites: a comparative study", Smart Structures and Materials, 2003. 
[33] J. Qiu, et. al., "Fabrication of piezoelectric ceramic fibers by extrusion of $\mathrm{Pb}(\mathrm{Zr}$, Ti)O3 powder and $\mathrm{Pb}(\mathrm{Zr}, \mathrm{Ti}) \mathrm{O} 3$ sol mixture", Smart Materials and Structures, Vol. 12, 331-337, 2003.

[34] W. K. Wilkie, et al., "Low-Cost Piezocomposite Actuator for Structural Control Applications", Proc. SPIE Vol. 3991, 2000.

[35] R. Brett Williams, et. Al., "Nonlinear Actuation Properties of Macro Fiber Composite Actuators" ASME Conf. Proc. 2003.

[36] D. Brei, B. J Cannon, "Piezoceramic Hollow Fiber Active Composites", Composites Science and Technology, Vol. 64, 2004.

[37] D. G. Lee, "Piezoelectric Hydraulic Pump with Innovative Active Valves", SPIE's 9th Annual International Symposium on Smart Structures and Materials, San Diego, California, Mar. 2002.

[38] K. Uchino, "Piezoelectric Actuators 2006", Journal of Electroceramics, Vol. 20, 301-311, 2008.

[39] J. J. Loverich, et. al., "A New Piezoelectric Actuator using a Feed-screw for Quasistatic Motion Accumulation" Journal of Intelligent Materials Systems and Structures, Vol. 19, 2008.

[40] C. A. Felippa, Lecture Notes: "Nonlinear Finite Element Methods - ASEN 6107", [Online Document, Available: http://www.colorado.edu/engineering/cas/courses.d/NFEM.d/, Accessed: Spring 2010] [41] T. R. Shrout, "Relaxor-Based Ferroelectric Single Crystals for Electro-Mechanical Actuators", Springer-Verlag, 1997. 
[42] P. P. Benham, P. J. Crawford, C. G. Armstrong, "Mechanics of Engineering Materials", Prentice Hall, 1996.

[43] M. A. Bhatti, "Advanced Topics in Finite Element Analysis of Structures: With Mathematica and MATLAB Computations", Wiley, 2006.

[44] M. Martinez, "Finite Element Model of Structures with Piezoelectric Elements", PhD Thesis, Department of Mechanical and Aerospace Engineering, Carleton University, 2006.

[45] L. Lapidus, G.F. Pinder, "Numerical Solution of Partial Differential Equations in Science and Engineering", John Wiley \& Sons, New York, 2002.

[46] G.R. Liu, S. S. Quek, "Finite Element Method: A Practical Course", ButterworthHeinemann, 2003.

[47] G. Dhondt, "CalculiX CrunchiX User's Manual, version 1.6", online document [Available: http://web.mit.edu/calculix_v1.6/CalculiX/ccx_1.6/doc/ccx/node14.html, accessed: Sept 2010]

[48] R. D. Cook, et. al., "Concepts and Applications of Finite Element Analysis", $4^{\text {th }}$ ed., Wiley, Madison, Wisconsin, 2001.

[49] M. A. Crisfield, "Nonlinear Finite Element Analysis of Solids and Structures", Wiley, West Sussex, England, 1991.

[50] H. MacNeal and R. L. Harder, "A proposed standard set of problems to test finite element accuracy", Finite element analysis and design 1, 1985.

[51] K. Y. Sze, el. al., "Popular Benchmark Problems for Geometric Nonlinear Analysis of Shells", Finite Elements in Analysis and Design 40, 1551-1569, 2004. 
[52] C. Park, et. al. "Bending and torsion models of beams with induced-strain actuators", Smart Materials and Structure, Vol5:98-113, 1996.

[53] M. S. Weinberg, "Working Equations for Piezoelectric Actuators and Sensors", ASME/IEEE Journal of MEMS, Vol 8, n4:71-78, 1999.

[54] V. K. Wickramasinghe, et al., "Design and Verification of a Smart Wing for an Extremely-Agile Micro-Air-Vehicle”, Journal of Smart Material Structures, 2011. 